\title{
Second Generation Tetrahydroquinoline-Based Protein Farnesyltransferase Inhibitors as Antimalarials
}

\author{
Pravin Bendale ${ }^{\dagger}$, Srinivas Olepu ${ }^{\dagger}$, Praveen Kumar Suryadevara ${ }^{\dagger}$, Vivek Bulbule $^{\dagger}$, Kasey \\ Rivas $\ddagger, \S$, Laxman Nallan ${ }^{\dagger}$, Brian Smart ${ }^{\dagger}$, Kohei Yokoyama ${ }^{\dagger}$, Sudha Ankala ${ }^{\dagger}$, Prakash Rao \\ Pendyala $\|$, David Floyd ${ }^{\perp}$, Louis J. Lombardo\#, David K. Williams ${ }^{\#}$, Frederick S. \\ tDepartment of Chemistry, University of Washington, Seattle, Washington 98195 \\ ¥Department of Medicine, University of Washington, Seattle, Washington 98195 \\ §Department of Pathobiology, University of Washington, Seattle, Washington 98195 \\ "Department of Molecular Biology and Microbiology, University of Central Florida, Orlando, Florida \\ 32826 \\ ${ }^{\perp}$ Pharmacopeia Drug Discovery, Princeton, New Jersey \\ \#Bristol-Myers Squibb Pharmaceutical Research Institute, Princeton, New Jersey 08543-4000 \\ ${ }^{\ominus}$ Department of Biochemistry, University of Washington, Seattle, Washington 98195
}

\begin{abstract}
Substituted tetrahydroquinolines (THQs) have been previously identified as inhibitors of mammalian protein farnesyltransferase (PFT). Previously we showed that blocking PFT in the malaria parasite led to cell death and that THQ-based inhibitors are the most potent among several structural classes of PFT inhibitors (PFTIs). We have prepared 266 THQ-based PFTIs and discovered several compounds that inhibit the malarial enzyme in the sub- to low-nanomolar range and that block the growth of the parasite ( $P$. falciparum) in the low-nanomolar range. This body of structure-activity data can be rationalized in most cases by consideration of the X-ray structure of one of the THQs bound to mammalian PFT together with a homology structural model of the malarial enzyme. The results of this study provide the basis for selection of antimalarial PFTIs for further evaluation in preclinical drug discovery assays.
\end{abstract}

\section{Introduction}

\begin{abstract}
New antimalarial drugs are needed because of widespread resistance to well-established agents such as chloroquine (www.mmv.org). We have been working on inhibitors of malaria protein farnesyltransferase $\left(\mathrm{PFT}^{\mathrm{a}}\right.$ ) because earlier studies showed that such agents are cytotoxic to Plasmodium falciparum, ${ }^{1-3}$ the causative agent of falciparum malaria. PFT catalyzes the
\end{abstract}

\footnotetext{
(C) 2007 American Chemical Society

* To whom correspondence should be addressed. Phone: 206-543-7142. Fax: 206-685-8665. gelb@chem.washington.edu. Note Added after ASAP Publication. This manuscript was released ASAP on August 28, 2007, with errors in the compound numbers in Schemes 5 and 7 and with an error in the structure for compound 119 in Table 2. The correct version was posted on September 13, 2007.

Supporting Information Available: HPLC traces of key compounds 6, 162, 110, 191, and 234. This material is available free of charge via the Internet at http://pubs.acs.org.
} 
transfer of the 15-carbon farnesyl group from farnesyl-pyrophosphate to the SH group of the tetrapeptide motif $\mathrm{C} a a \mathrm{X}$ (C is cysteine, $a$ is usually but not necessarily an aliphatic amino acid, and $\mathrm{X}$ is a variety of amino acids) present at the $\mathrm{C}$-terminus of proteins that become farnesylated. Inhibitors of mammalian PFT have been extensively developed as anticancer agents by the pharmaceutical industry, ${ }^{4}$ and thus, we have the opportunity to extend the medicinal chemistry and preclinical pharmacology of PFT inhibitors toward the development of antimalarial drugs (piggy-back drug development). To this end, we tested many of the known classes of PFT inhibitors and found that tetrahydroquinoline (THQ)-based PFT inhibitors developed at Bristol Myers Squibb ${ }^{5}$ are the most potent against $P$. falciparum PFT (Pf-PFT) and against parasite growth in human red blood cells. ${ }^{3}$ Our previous medicinal chemistry efforts led to the identification of THQ 1 (Figure 1) and a few related compounds that inhibit Pf-PFT in vitro, with $\mathrm{IC}_{50}$ (concentration of inhibitor that 50\% inhibits Pf-PFT) values of $\sim 0.6$ $\mathrm{nM}$, and inhibit parasite growth in red cells with $\mathrm{ED}_{50}$ (concentration of inhibitor that $50 \%$ inhibits the growth of $P$. falciparum in red blood cells in vitro) values of $\sim 5 \mathrm{nM} .{ }^{3}$ Studies with mammalian PFT have shown that the 6-cyano group on the THQ ring is important in conferring tight enzyme binding, ${ }^{6}$ and structural studies show that the imidazole appended to $\mathrm{N}-1$ of the THQ ring directly coordinates the $\mathrm{Zn}^{2+}$ ion at the active site of mammalian PFT. ${ }^{7,8}$

Continuous dosing of THQ 1 using surgically implanted, osmotic minipumps in mice infected with rodent malaria (P. berghei) led to a dramatic reduction in parasite number. ${ }^{3}$ Studies of malaria resistance to these Pf-PFT inhibitors provided very strong evidence that blocking PfPFT action is the basis for parasite killing. ${ }^{7,8}$ The reasons that PFT inhibitors are very toxic to malarial cells but not to mammalian cells are not known. One hypothesis is that the malaria parasite appears to lack protein geranylgeranyltransferase type I, the enzyme responsible for the monogeranylgeranylation of proteins in mammalian cells. Thus, some critical housekeeping proteins may be farnesylated in malaria but geranylgeranylated in mammalian cells.

The purpose of the present study is to report our continued efforts to develop THQ-based antimalarials. A number of THQs were prepared with various groups attached to the sulfonyl group of $\mathbf{1}\left(\mathrm{R}_{1}\right.$ of THQ $\mathbf{2}$ in Figure 1) or to the sulfonamide nitrogen $\left(\mathrm{R}_{2}\right.$ in THQ 2 in Figure 1). Less extensive structure-activity studies were carried out by variation of other groups attached to the THQ core. We were able to identify several new THQ-based Pf-PFT inhibitors with sub- to low-nanomolar potency on Pf-PFT and with low nanomolar potency on $P$. falciparum growth in vitro.

\section{Chemistry}

Many of the THQs 2 prepared in this study were prepared by the route shown in Scheme 1 starting from racemic 6-cyano-3-amino-THQ (compound $\mathbf{3}$, Scheme 1), which was made as described. ${ }^{5}$ This route is useful for variation of the $\mathrm{R}_{2}$ group, which is added in the last synthetic step. Scheme 2 was used to prepare analogs of $\mathbf{2}$ in which the $\mathrm{R}$ group attached to the piperidine nitrogen is varied. Scheme 3 was used to allow easier variation of both the $R_{1}$ and $R_{2}$ groups because, unlike in Scheme 1, the $R_{1}$ group is introduced later in the synthesis. Scheme 4 was used to prepare THQs, which lack the methyl group on the zinc-binding imidazole. In this case, tritylation of the imidazole was required prior to alkylation of the sulfonamide nitrogen.

Scheme 5 shows the synthesis of a THQ analog in which the methylene bridge between the $\mathrm{N}-1$ of the THQ core and the $\mathrm{Zn}^{2+}$-binding imidazole group is replaced with a sulfonyl group or with a $\mathrm{CH}\left(\mathrm{CH}_{3}\right)$ group. THQs containing a 6-phenyl group in place of the 6-cyano group were prepared according to Scheme 6. The key step is the introduction of the phenyl group via

Abbreviations: ED50, effective dose of inhibitor that reduces parasite growth in vitro by 50\%; IC50, inhibitor concentration that reduces the rate of PFT in vitro by 50\%; PFT, protein farnesyltransferase, PFTI, PFT inhibitor, THQ, tetrahydroquinoline. 
Suzuki coupling (conversion of $\mathbf{2 1}$ to 22). Scheme 7 shows the synthesis of THQ analogs in which the 6-CN is replaced with carbonyl-bearing functional groups. Scheme 8 was used to prepare the THQ analog 34 . The key reaction is nucleophilic displacement between the mesylate derived from the indicated secondary alcohol $\mathbf{3 2}$ and secondary amine $\mathbf{3 3}$.

\section{Inhibition of Pf-PFT and $\boldsymbol{P}$. falciparum Growth by THQ-Based Pf-PFT Inhibitors}

We first give a general description of the potencies of THQ-based Pf-PFT inhibitors on the enzyme and on $P$. falciparum growth in vitro, and then we attempt to provide a structural rational for some of the activity data. Our initial structure-activity data on THQ-based inhibitors of Pf-PFT led to the discovery of compounds with $\mathrm{R}_{1}=2$-pyridyl or $\mathrm{N}_{1}$-methyl-4imidazolyl as being potent inhibitors of Pf-PFT. ${ }^{3}$ In Table 1, we report a study of THQs with $\mathrm{R}_{1}=2$-pyridyl and with a variety of substituents as $\mathrm{R}_{2}$ groups. A number of THQs were found that inhibited Pf-PFT in vitro by $50 \%\left(\mathrm{IC}_{50}\right)$ in the low nanomolar range and that also inhibited the growth of $P$. falciparum in erythrocyte cultures in vitro by $50 \%\left(\mathrm{ED}_{50}\right)$ in the low nanomolar range (i.e., 48, 55, 56, 57, 61, and 62). The most potent compound in the series is 55 with an $\mathrm{ED}_{50}=17 \mathrm{nM}$ for the 3D7 strain and $10 \mathrm{nM}$ for the $\mathrm{K} 1$ strain. Well-established antimalarial drugs such as chloroquine display $\mathrm{ED}_{50}$ values in the low nanomolar range. Thus, the potency achieved for some of our THQ-based PFT inhibitors is probably sufficient for an antimalarial drug discovery effort. In general, we did not find any compound that inhibited P. falciparum growth in the low nanomolar range that was a relatively poor inhibitor of Pf-PFT.

Table 2 summarizes results for THQ-based PFT inhibitors with $\mathrm{R}_{1}=\mathrm{N}_{1}$-methyl-4-imidazolyl and with variation of the $\mathrm{R}_{2}$ group. Many compounds were found with $\mathrm{IC}_{50} \mathrm{~s}$ and $\mathrm{ED}_{50} \mathrm{~s}$ in the low nanomolar range. The most potent in the series are 106, 107, 102, 104, 115, and 131 with values of $\mathrm{ED}_{50}<10 \mathrm{nM}$. Compound 106 shows exceptional potency with an $\mathrm{ED}_{50}=1.8 \mathrm{nM}$. This is the most potent compound in terms of $\mathrm{ED}_{50}$ that we found in the current study and among the most potent antimalarials ever reported. In general, the compounds with $\mathrm{R}_{1}=\mathrm{N}_{1^{-}}$methyl-4-imidazolyl are more potent than the analogous compounds with $\mathrm{R}_{1}=2$-pyridyl.

We also carried out side-by-side pharmacokinetic studies of potent THQ-based Pf-PFT inhibitors to discover a compound that could be tested in malaria-infected rodents. ${ }^{9} \mathrm{We}$ measured the rate of flux of THQ-based PFT inhibitors across a tight-junction monolayer of Caco-2 cells and showed that this in vitro assay is a good predictor of oral bioavailability of these compounds in rodents. ${ }^{9}$ In general, we found that $\mathrm{R}_{1}=2$-pyridyl promotes better Caco- 2 permeability and oral bioavailability than compounds with $\mathrm{R}_{1}=\mathrm{N}_{1}$-methyl-4-imidazolyl. ${ }^{9}$ Because it appears that THQ-based PFT inhibitors are cleared in rodents by hepatic metabolism, we measured the half-time for metabolism of THQs by rat and mouse liver microsomes in vitro. ${ }^{9}$ Based on an overall balance of desirable properties, 6 emerged as a promising antimalarial lead compound. ${ }^{9}$ Thus, we investigated in detail the structure-activity relationships among $\mathbf{6}$ analogs. Table 3 shows the results with $\mathbf{6}$ analogs in which the R group attached to the piperidine $\mathrm{N}$ of the $\mathrm{R}_{2}$ group is varied. In general, we found a good correlation between $\mathrm{IC}_{50}$ values for Pf-PFT inhibition and $\mathrm{ED}_{50}$ values for blocking the growth of parasites in vitro.

When those compounds in Table 3 with values of $\mathrm{ED}_{50}<\sim 100 \mathrm{nM}$ were tested for Caco-2 permeability and microsomal stability, ${ }^{9} \mathbf{1 6 2}$ emerged as a compound with a good balance of potency and desirable pharmacokinetic properties. In Table 4, we summarize results with compounds in which $\mathrm{R}_{2}$ is held as the $\mathrm{R}_{2}$ of $\mathbf{1 6 2}$ and $\mathrm{R}_{1}$ is varied. We also studied compounds with $\mathrm{R}_{2}=2$-fluorophenylCH $2,4-\mathrm{MeSO}_{2}$-phenylCH $\mathrm{CH}_{2}$, and $t-\mathrm{BuNHCOCH}_{2}$ because these groups led to potent compounds in the early scans. Most compounds in this series were significantly less potent on Pf-PFT and on parasites compared to 162. The exceptions were those with a small heterocyclic $\mathrm{R}_{1}$ group, that is, $\mathbf{1 9 1}$. 
Molecular modeling studies described below suggested that addition of a Me group to $\mathrm{R}_{1}=$ $\mathrm{N}_{1}$-methyl-4-imidazolyl at the 5-position would better fill the $\mathrm{R}_{1}$-binding pocket on Pf-PFT. Table 5 shows a scan of $\mathrm{R}_{2}$ groups keeping $\mathrm{R}_{1}$ as 4 - $\left(\mathrm{N}_{1}\right.$-methyl-5-Me-imidazolyl). Compound $\mathbf{2 3 4}$ with the same $\mathrm{R}_{2}$ as in $\mathbf{1 6 2}$ emerged as the best compound.

Table 6 gives the activity of compounds in which the sulfonyl is replaced by carbonyl (sulfonamide to amide change). In general, these compounds are much poorer inhibitors of PfPFT and of $P$. falciparum growth than the corresponding sulfonyl-containing THQ compounds. Thus, we prepared only a limited set of these amide-containing THQ compounds.

Table 7 shows THQ-based compounds with alteration to the $\mathrm{N}$-Me-imidazole $\mathrm{CH}_{2}$ group attached to the THQ ring N. This substituted imidazole is a direct ligand to the $\mathrm{Zn}^{2+}$ at the active site of Pf-PFT (see below). Removal of the Me group from the imidazole has only a minimal effect on binding to Pf-PFT (as predicted by modeling) but greatly increases $\mathrm{ED}_{50}$ for killing parasites, suggesting that methylation of the $\mathrm{Zn}^{2+}$-binding imidazole promotes penetration of compound across erythrocyte and parasite membranes. Replacement of the $\mathrm{CH}_{2}$ that links the imidazole to the THQ ring $\mathrm{N}$ by $\mathrm{SO}_{2}$ obliterates binding to Pf-PFT and antiparasite activity. Addition of a Me group to this $\mathrm{CH}_{2}$ bridge (mixture of four stereoisomers) also greatly reduces activity as does replacement of the imidazole by a 3-pyridylCH $\mathrm{CH}_{2}$ group.

THQ compounds with a 6-phenyl instead of 6-CN were available to us from a different drug discovery program. Some of these compounds maintain modest potency as inhibitors of PfPFT and as antimalarials $(\mathbf{2 6 6}, \mathbf{2 6 7})$, but in general, we could not find a 6-phenyl THQ with a potency approaching our best compounds in the 6-CN-THQ series. It may be noted that the length of the phenyl is about the same as the length of the $\mathrm{CN}$ group, and thus, compounds like 267 may be binding to Pf-PFT in the same way as the 6-CN-THQ compounds, but we have no experimental evidence for this.

The data in Table 9 shows that replacement of the 6-CN group on the THQ core with various amides or a carboxylate is very detrimental to Pf-PFT binding and parasite killing.

\section{Structural Rational of the Structure-Activity Results for THQ-Based PFT Inhibitors}

To date, we have not been able to obtain Pf-PFT in amounts sufficient for crystallization trials. However, the X-ray structure of rat and human PFTs are available alone and with a number of bound inhibitors, including the structure of the rat-PFT/162 complex. ${ }^{8}$ As described in General Methods, we made a homology model of Pf-PFT based on this experimental structure. Most of the residues in the $\mathbf{1 6 2}$ binding site are conserved between rat- and Pf-PFT. The only differences are that Pro- $150 \beta$ of rat-PFT, which resides in the vicinity of the terminal methyl of the $\mathrm{R}_{2}$ carbamate of 162, is replaced with Thr-512 $\beta$ in Pf-PFT. Tyr-93 $\beta$, adjacent to the 6$\mathrm{CN}$ of 162, is Leu-443 $\beta$ in Pf-PFT. Two more conservative changes, somewhat further away from 162, play a role in the binding of other THQ-based inhibitors discussed in this paper: Tyr-166 $\alpha$ (Phe-151 $\alpha$ in Pf-PFT) and Phe-360 $\beta$ (Tyr-836 $\beta$ in Pf-PFT). Thus, the crystal structure of the complex of $\mathbf{1 6 2}$ with the rat enzyme is of great value despite only $23 \%$ sequence identity for rat- versus Pf-PFT $\alpha$-subunits and $37 \%$ identity between the $\beta$-subunits.

The elucidation of the structure-activity relationships of $\mathbf{1 6 2}$ and other THQ-based PFT inhibitors is complicated by the use of racemates. Each compound is a mixture of two enantiomers due to the chiral center at carbon-3 of the THQ ring (Figure 1). Moreover, each enantiomer can exist in two conformations, with the 3-substituent either axial or equatorial. Hence, it is crucial to know which of the two enantiomers block the enzyme. To gain insight, we first determined the $\mathrm{IC}_{50}$ of the individual enantiomers of 162. This was carried out by coupling 6-cyano-1,2,3,4-tetrahydro-quinolin-3-ylamine hydrochloride to $S$-mandelic acid and separating the diastereomers as described. ${ }^{5,10}$ The two enantiomeric amines were then 
converted to the two enantiomers of $\mathbf{1 6 2}, \mathbf{1 6 3}$, and $\mathbf{1 6 4}$, as shown in Scheme 1 . The $R$ enantiomer of 162, 163, displayed an $\mathrm{IC}_{50}$ against Pf-PFT that is 10 times below that of the $S$-enantiomer, 164 (Table 3). This difference is recapitulated in the observed $\mathrm{ED}_{50 \mathrm{~S}}$ for blocking $P$. falciparum growth. The 10 -fold difference in enzyme binding is modest, corresponding to a difference in binding energy of $1.3 \mathrm{kcal} / \mathrm{mol}$. Superposition of the two enantiomers shows that the first substituent atoms of the two possible equatorial enantiomers at C3 of the THQ ring are only $0.8 \AA$ apart, thereby, allowing for the C3 substituents to adopt similar positions in the binding site regardless of the stereochemistry (Figure 2).

Figure 3 (top panels) shows the experimental, ternary structure of rat-PFT with bound 162 inhibitor and farnesyl-pyrophosphate substrate. ${ }^{8}$ The THQ ring of $\mathbf{1 6 2}$ stacks face-on-face and at an angle of about $25^{\circ}$ with Tyr $361 \beta$ (Tyr $837 \beta$ in the Pf-PFT homology model), projecting the $N$-methyl-imidazole so that it coordinates the catalytic $\mathrm{Zn}^{2+}$ of the enzyme (Figure 3 ). The $6-\mathrm{CN}$ sits in a narrow groove made by Leu-96 (Leu $446 \beta$ in Pf-PFT)), Trp-106 $\beta$ (Trp-456 $\beta$ in Pf-PFT), Asp-359 $\beta$ (Asp-835 $\beta$ in Pf-PFT), and Tyr-361 $\beta$ (Tyr-837 $\beta$ in Pf-PFT). In this way, the 6-CN-benzo group of $\mathbf{1 6 2}$ adopts a similar but not identical position as the group of 3benzyl-1-(3H-imidazol-4-ylmethyl)-4-(thiophene-2-sulfonyl)-2,3,4,5-tetrahydro- $1 H$-benzo [e][1,4]-diazepine-7-carbonitrile (BMS-214662), a tetrahydrobenzodiazepine-based PFT inhibitor for which an X-ray structure of its complex with rat PFT has been published; ${ }^{11}$ the difference in 6-CN-benzo group atoms ranges from 0.5 to $0.9 \AA$ (Figure 3 , bottom left panel). The X-ray structure of bound $\mathbf{1 6 2}$ (obtained by soaking the rat PFT crystals with racemic 162) shows that only one enantiomer binds; the data is consistent with our observation that the enantiomer 163 is the better inhibitor (see above). The C3 substituent is equatorial, reflecting the $2.7 \mathrm{kcal} / \mathrm{mol}$ calculated preference for this conformation over the axial one in solution. The 2 -pyridyl $\mathrm{R}_{1}$ substituent makes a hydrogen bond with Tyr-361 $\beta$ (Tyr-837 $\beta$ in Pf-PFT) and stacks against the second isoprene unit of farnesyl-pyrophosphate. The 2-pyridyl also stacks against the $\mathrm{Zn}^{2+}$-binding $N$-Me-imidazole of $\mathbf{1 6 2}$. The $\mathrm{R}_{2}$ substituent resides in a large, mainly hydrophobic pocket, which is normally occupied by the side chain of the $\mathrm{X}$ residue of the CaaX motif of proteins that are farnesylated (Figure 3, bottom right panel). The $\mathrm{R}_{2}$ group only partially fills this pocket. The carbonyl oxygen of the carbamate portion of the $\mathrm{R}_{2}$ substituent of 162 accepts hydrogen bonds with the side chains of Ser-99 $\beta$ and Trp-102 $\beta$ of rat-PFT (Ser-449 $\beta$ and Trp-452 $\beta$ in Pf-PFT). The terminal Me of the carbamate portion of the $\mathrm{R}_{2}$ group

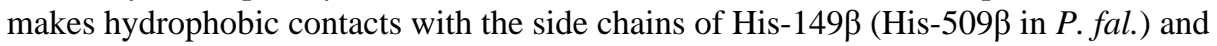
Ala-151 $\beta$ (Ala-511 $\beta$ in Pf-PFT). The $\mathrm{R}_{2}$ piperidine contacts Trp-102 $\beta$ (Trp-452 $\beta$ in Pf-PFT). Remarkably, the two oxygens of the sulfonamide do not make direct hydrogen bonds to the protein.

We used the 162/PFT structural information as a starting point for constructing models of many of the other THQ-based PFT inhibitors prepared in this paper. Rather than discuss each compound, we describe the trends that are supported by the structural information. Figure 4 (top panel) shows the predicted binding mode of $\mathbf{1 6 2}$ in the active site of Pf-PFT. As already noted, THQ-based compounds in Table 1 have a 6-CN group, $\mathrm{R}_{1}=2$-pyridyl, and variation of the $\mathrm{R}_{2}$ group. Most of the compounds in Table 1 are predicted to be able to bind with $\mathrm{R}_{2}$ in the $a_{2} \mathrm{X}$ region of the $\mathrm{C} a_{1} a_{2} \mathrm{X}$ pocket, while maintaining the pose of the THQ scaffold and $\mathrm{R}_{1}$ and $\mathrm{R}_{3}$ groups as seen experimentally. $\mathrm{R}_{2}$ groups that end in a polar group are poor Pf-PFT binders because they cannot interact favorably with the largely hydrophobic environment $(\mathbf{3 8}, \mathbf{3 9}, \mathbf{5 2}$, $\mathbf{5 3}, \mathbf{5 4}, \mathbf{9 0})$. Moreover, THQs with small $\mathrm{R}_{2}$ groups $(\mathbf{3 8}, \mathbf{5 2}, \mathbf{5 3}, \mathbf{9 0})$ barely fill the pocket and therefore display poor enzyme inhibition. On the other hand, the $\mathrm{R}_{2}$ group of $\mathbf{8 7}$ is too large to fill the pocket. Long, linear $\mathrm{R}_{2}$ groups, some with a small branch, that provide several apolar atoms work better as Pf-PFT inhibitors as they can reach Trp-456 $\beta$, Trp-452 $\beta$, Ala-511 $\beta$ and His-509 $\beta$, but this comes at a cost of the loss in conformational entropy when the flexible side chain rigidifies in the active site of Pf-PFT $(35,36,40,41,42,43,44,45,48,46,47,49,50$, 38). 
The $\mathrm{R}_{2}$ groups of all other THQs contain one or more rings. The best $\mathrm{R}_{2}$ groups have a short ethylene linker (thus minimizing entropic cost of enzyme binding) followed by a fivemembered, aromatic heterocycle $(\mathbf{5 5}, \mathbf{5 6}, \mathbf{5 7})$. They are predicted to bind in the same way as 162 (Figure 4, top right), engaging in extensive hydrophobic interactions with Leu-446 $\beta$, Trp-456 $\beta$, Trp- $452 \beta$, and C4 of their own THQ scaffold; the latter probably stabilizes the enzyme-bound $\mathrm{R}_{2}$ conformation for the inhibitor free in solution. Two of the $\mathrm{R}_{2}$ ring atoms remain solvent exposed, which explains why those two positions can be replaced by nitrogen atoms $(\mathbf{5 6}, \mathbf{5 7})$. However, a slightly larger benzene ring cannot be accommodated in the same

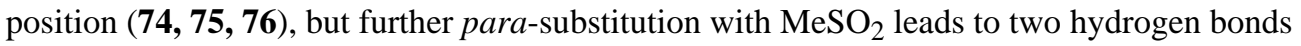
of this group with Arg-564 $\beta$ and Gln-152 $\alpha$, plus hydrophobic contacts with His-509 $\beta$ (69), explaining the decent potency of this compound. Expansion of the pyrrole ring to an indole (64) leads to reduced inhibition because the $\mathrm{R}_{2}$ conformation cannot be maintained due to steric clash with Leu-4463. Expansion of the pyrrole ring to phthalimide (85) also leads to a different conformation because of steric reasons, though the benzo part is now predicted to interact with His-509ß.

Most $\mathrm{R}_{2}$ groups with a single methylene linker to an aromatic ring are unable to make an efficient contact with the hydrophobic residues in the pocket of Pf-PFT as the ethylene-linked ones, even when they in turn carry a hydrophobic substituent $(\mathbf{5 8}, \mathbf{5 9}, \mathbf{6 0}, \mathbf{6 1}, \mathbf{6 2}, \mathbf{6 3}, \mathbf{6 5}, \mathbf{6 6}$,

$68,70,71,69,73,77,78,79,80,81,83,6,84)$. Longer linkers to a ring also do not allow for efficient contact of hydrophobic residues in the pocket $(\mathbf{8 2}, \mathbf{8 6}, \mathbf{8 8})$.

Replacement of the sulfonamide group of THQs with amides universally leads to a loss of binding to Pf-PFT (Table 6). In the crystal structure of rat PFT with 162, and by homology with Pf-PFT, the sulfonamide moiety adopts a known low-energy conformation. Sulfonamides, which have a pyramidalized nitrogen atom, adopt two low-energy conformations because the $\mathrm{N}-\mathrm{S}$ bond has partial double character. One is eclipsed, thereby allowing for maximal overlap of the nitrogen lone pair electrons and the $\mathrm{S}=\mathrm{O}$ bonds (Figure 5). The other one is obtained by nitrogen inversion, thereby reducing the steric interactions. ${ }^{12} \mathrm{Ab}$ initio calculations at the MP2/6-31+G* level for the model compound $N, N$-dimethylmethanesulfonamide, the closest analog to the sulfonamide substructure in our molecules, shows that this compound prefers the inverted conformation with a $\mathrm{C}-\mathrm{N}-\mathrm{S}=\mathrm{O}$ torsion angle of $30^{\circ} . .^{13}$ This conformation is similar to what is observed in the X-ray structure of $\mathbf{1 6 2}$ bound to rat PFT (Figure 5). Simple stereochemical considerations indicate why the amide analogs of our sulfonamide THQ PFTIs are poor inhibitors. The amide function has two trigonal centers, and thus, a planar arrangement of all substituents. This leads to an orientation of the $\mathrm{R}_{1}$ group in the amides that is $90^{\circ}$ off from the orientation of the $\mathrm{R}_{1}$ of $\mathbf{1 6 2}$ (Figure 5), and this altered orientation is sterically disallowed by the active site of the PFT.

Modeling showed that there is space in the active site of Pf-PFT to accommodate groups other than $N$-methyl on the $\mathrm{Zn}^{2+}$-binding imidazole. A benzyl (254, Table 7) was not very effective probably because of the extra entropy loss due to two extra rotatable bonds. Replacement of the methylene linker in the $\mathrm{Zn}^{2+}$-binding arm (attached to $\mathrm{N} 1$ of the THQ ring) by an $\mathrm{SO}_{2}$ (255, Table 7) leads to an inactive compound. This is most likely because of the increased electron-withdrawing character of the $\mathrm{SO}_{2}$ linker, thereby decreasing the basicity of the imidazole $\mathrm{N}$ and, thus, the coordination affinity to the active site $\mathrm{Zn}^{2+}$. Using the SPARC v3.1 program, ${ }^{14,15}$ we calculated that the $\mathrm{p} K_{\mathrm{a}}$ of the protonated imidazole drops from 6.8 to 5.1 when the $\mathrm{CH}_{2}$ linker is replaced with $\mathrm{SO}_{2}$. If the drop in proton affinity is the same for $\mathrm{Zn}^{2+}$, this drop in $\mathrm{p} K_{\mathrm{a}}$ of 1.7 would give a drop in $\mathrm{IC}_{50}$ by 50 -fold.

Replacement of the 6-CN group with phenyl is tolerated (Table 8). Replacement with more polar substituents (Table 9) is not tolerated, which is consistent with the lack of apporpriate hydrogen bond donors and acceptors in the pocket on Pf-PFT that binds the 6-CN group. 


\section{Inhibition of Mammalian PFT}

A subset of THQ-based PFTIs were tested for inhibition of rat PFT, and results are summarized in Table 10 and compared to those obtained with Pf-PFT. Most compounds displayed comparable potency on both enzymes. The exceptions are $\mathbf{8 4}$ and $\mathbf{1 2 9}$, which are about 10-fold more potent on Pf-PFT than on rat PFT, and 126, which shows the reverse preference. As noted in our earlier publications, clinical trials have shown that PFTIs are well tolerated in man after several weeks of continuous dosing. Thus, specificity toward Pf-PFT versus mammalian PFT is probably not required for a drug that would be used to treat malaria over the course of a few days.

\section{Conclusions}

In this study we show that THQ-based PFTIs are potent inhibitors of Pf-PFT activity and of erythrocytic stage $P$. falciparum growth. Several compounds were found with growth inhibition potency down in the low nanomolar range, with several compounds blocking parasite growth at concentrations $<5 \mathrm{nM}$. A good deal of the structure-activity data for the inhibition of Pf-PFT can be accounted for based on the structural consideration of one of the compounds, 162, bound to mammalian PFT. In a companion study, we have carried out detailed preclinical pharmacokinetic studies of our most potent Pf-PFT inhibitors. ${ }^{9}$ Together, these two studies provide the basis for further development of Pf-PFT inhibitors as novel antimalarial drugs.

\section{Experimental Section}

\section{Synthesis of Compounds. General Methods}

Unless otherwise indicated, all anhydrous solvents were commercially obtained and stored under nitrogen. Reactions were performed under an atmosphere of dry nitrogen in oven-dried glassware and were monitored for completeness by thin layer chromatography (TLC) using silica gel 60 F-254 $(0.25 \mathrm{~mm})$ plates with detection with UV light. ${ }^{1} \mathrm{H}$ NMR spectra were recorded on dilute solutions in $\mathrm{CDCl}_{3}, \mathrm{CD}_{3}$-OD, or DMSO- $d_{6}$ at 300 or $500 \mathrm{MHz}$. Chemical shifts are reported in parts per million $(\delta)$ downfield from tetramethylsilane (TMS). Coupling constants $(J)$ are reported in Hz. Electrospray ionization mass spectra were acquired on an Bruker Esquire LC00066. Flash chromatography was carried out with silica gel $(40-63 \mu \mathrm{m})$. Preparative reverse phase HPLC was performed on an automated Varian Prep star system using a gradient of $20 \% \mathrm{MeOH}$ to $100 \% \mathrm{MeOH}$ (with $0.1 \%$ trifluoroacetic acid) over 30 min using a YMC S5 ODS column $(20 \times 100 \mathrm{~mm}$, Waters, Inc.). All final compounds (those tested on Pf-PFT and on parasites cultures) were purified by HPLC as above to single eluting peaks.

\section{General Procedure for Synthesis of Compounds According to Scheme 1. Sulfonation}

A solution of 6-cyano-1,2,3,4-tetrahy-dro-quinolin-3-ylamine hydrochloride 3 (5 mmol), sulfonyl chloride (10 mmol; for example, ref 16 , and $N, N$-diisopropylethyl amine ( $15 \mathrm{mmol}$ ) in $25 \mathrm{~mL}$ of anhydrous $\mathrm{CH}_{3} \mathrm{CN}$ was stirred at room temperature overnight. A light-colored precipitate of $\mathbf{4}$ was isolated by vacuum filtration. More often, product $\mathbf{4}$ was obtained by flash chromatography on a silica gel column, eluting with 50\% ethyl acetate/hexane: yields 85-95\%.

1-Methyl-1 H-imidazole-4-sulfonic Acid (6-Cyano-1,2,3,4-tetrahydro-quinolin-3-yl)-amide (4)

${ }^{1} \mathrm{H}$ NMR $\left(500 \mathrm{MHz}\right.$, DMSO- $\left.d_{6}\right) \delta 7.80(\mathrm{~s}, 1 \mathrm{H}), 7.78(\mathrm{~s}, 1 \mathrm{H}), 7.70(\mathrm{br} \mathrm{s}, 1 \mathrm{H}), 7.25(\mathrm{~d}, J=8.7$ $\mathrm{Hz}, 1 \mathrm{H}), 7.20(\mathrm{~s}, 1 \mathrm{H}), 6.81(\mathrm{~s}, 1 \mathrm{H}), 6.51(\mathrm{~d}, J=8.6 \mathrm{~Hz}, 1 \mathrm{H}), 3.78(\mathrm{~s}, 3 \mathrm{H}), 3.52-3.43(\mathrm{~m}, 1 \mathrm{H})$, 3.25-3.20 (m, 1H), 3.15-2.9 (m, 1H), 2.81-2.73 (m, 1H), 2.65-2.53 (m, 1H). MS m/z 318.5 $\left(\mathrm{M}+\mathrm{H}^{+}\right)$. 
Pyridine-2-sulfonic Acid (6-Cyano-1,2,3,4-tetrahydro-quinolin-3-yl)-amide (4)

${ }^{1} \mathrm{H}$ NMR $\left(300 \mathrm{MHz}\right.$, methanol- $\left.d_{4}\right) \delta 8.51(\mathrm{~d}, J=7.2 \mathrm{~Hz}, 1 \mathrm{H}), 8.02(\mathrm{td}, J=2.1,7.8 \mathrm{~Hz}, 1 \mathrm{H})$, $7.92(\mathrm{dt}, J=1.8,7.5 \mathrm{~Hz}, 1 \mathrm{H}), 7.47(\mathrm{ddd}, J=1.2,4.8,7.5 \mathrm{~Hz}, 1 \mathrm{H}), 7.12(\mathrm{dd}, J=2.1,8.4 \mathrm{~Hz}$, $1 \mathrm{H}), 7.0(\mathrm{~d}, J=1.8 \mathrm{~Hz}, 1 \mathrm{H}), 6.3(\mathrm{~d}, J=8.4 \mathrm{~Hz}, 1 \mathrm{H}), 5.87(\mathrm{~d}, J=7.8 \mathrm{~Hz}, 1 \mathrm{H}), 3.94-4.01(\mathrm{~m}$, $1 \mathrm{H}), 3.36(\mathrm{dd}, J=2.7,12 \mathrm{~Hz}, 1 \mathrm{H}), 3.25(\mathrm{ddd}, J=2.1,4.5,12.3 \mathrm{~Hz}, 1 \mathrm{H}), 2.87(\mathrm{dd} J=4.2,16.5$ $\mathrm{Hz}, 1 \mathrm{H}), 2.71$ (ddd, $J=2.1,4.8,16.5 \mathrm{~Hz}, 1 \mathrm{H})$. MS m/z $315\left(\mathrm{M}+\mathrm{H}^{+}\right)$.

\section{Reductive Amination}

A mixture of sulfonamide 4 (5 mmol), 1-methyl- $1 \mathrm{H}$-imidazole-5-carboxaldehyde (10 mmol), 16 and $20 \mathrm{~mL}$ of $50 \%$ trifluoroacetic acid in dichloroethane was warmed at $50{ }^{\circ} \mathrm{C}$ under argon. After $2 \mathrm{~h}$, triethylsilane $(20 \mathrm{mmol})$ was added. After $48 \mathrm{~h}$, the solvent was removed under reduced pressure, and the crude product was partitioned between methylene chloride and $1 \mathrm{~N}$ $\mathrm{NaOH}(45 \mathrm{~mL})$. The organic layer was separated, and the aqueous layer was extracted with $\mathrm{CH}_{2} \mathrm{Cl}_{2}(3 \times 25 \mathrm{~mL})$. The combined organic layers were dried over $\mathrm{MgSO}_{4}$, filtered, and concentrated. The crude residue was either recrystallized with dichloromethane or purified on a flash silica gel chromatography to afforded 5 in $45-50 \%$ yield.

\section{1-Methyl-1 $\mathrm{H}$-imidazole-4-sulfonic Acid [6-Cyano-1-(3-methyl-3H-imidazol-4- ylmethyl)-1,2,3,4-tetrahydro-quinolin-3-yl]-amide Trifluoro Acetate Salt (5)}

Recrystallized from $\mathrm{CH}_{2} \mathrm{Cl}_{2}$ afforded the product as a white solid (70\%). ${ }^{1} \mathrm{H} \mathrm{NMR}(300 \mathrm{MHz}$, methanol- $\left.d_{4}\right) \delta 8.89(\mathrm{~s}, 1 \mathrm{H}), 7.82(\mathrm{~s}, 1 \mathrm{H}), 7.72(\mathrm{~s}, 1 \mathrm{H}), 7.41(\mathrm{~s}, 1 \mathrm{H}), 7.36(\mathrm{~d}, J=8.2 \mathrm{~Hz}, 1 \mathrm{H}$,), $7.22(\mathrm{~s}, 1 \mathrm{H}), 6.80(\mathrm{~d}, J=8.6 \mathrm{~Hz}, 1 \mathrm{H}), 4.80(\mathrm{~d}, J=16.80 \mathrm{~Hz}, 1 \mathrm{H}), 4.65(\mathrm{~d}, J=16.80 \mathrm{~Hz}, 1 \mathrm{H})$, 3.87 (s, 3H), 3.85-3.79 (m, 1H), 3.78 (s, 3H), 3.55 (dd, $J=11.9 \mathrm{~Hz}, 1 \mathrm{H}), 3.42$ (dd, $J=12.4$ $\mathrm{Hz}, 1 \mathrm{H}), 3.05(\mathrm{dd}, J=15.78 \mathrm{~Hz}, 1 \mathrm{H}), 2.71(\mathrm{dd}, J=15.78 \mathrm{~Hz}, 1 \mathrm{H}) . \mathrm{MS} m / z 412.5\left(\mathrm{M}+\mathrm{H}^{+}\right)$.

\section{Pyridine-2-sulfonic Acid [6-Cyano-1-(3-methyl-3H-imidazol-4-ylmethyl)-1,2,3,4-tetrahydro- quinolin-3-yl]-amide (5)}

${ }^{1} \mathrm{H}$ NMR $\left(300 \mathrm{MHz}\right.$, DMSO- $\left.d_{6}\right) \delta 8.70(\mathrm{~d}, J=4.8 \mathrm{~Hz}, 1 \mathrm{H}), 8.14(\mathrm{~d}, J=6.6 \mathrm{~Hz}, 1 \mathrm{H}), 8.06(\mathrm{dt}$, $J=1.8,7.5 \mathrm{~Hz}, 1 \mathrm{H}), 7.99$ (s, $1 \mathrm{H}), 7.67$ (ddd, $J=1.2,4.8,7.8 \mathrm{~Hz}, 1 \mathrm{H}), 7.37$ (dd, $J=2.1,8.7$ $\mathrm{Hz}, 1 \mathrm{H}), 7.27(\mathrm{~d}, J=1.8 \mathrm{~Hz}, 1 \mathrm{H}), 6.88(\mathrm{~s}, 1 \mathrm{H}), 6.8(\mathrm{~d}, J=8.7 \mathrm{~Hz}, 1 \mathrm{H}), 4.62(\mathrm{~d}, J=16.5 \mathrm{~Hz}$, $1 \mathrm{H}), 4.59(\mathrm{~d}, J=16.5 \mathrm{~Hz}, 1 \mathrm{H}), 3.73-3.85(\mathrm{~m}, 1 \mathrm{H}), 3.60(\mathrm{~s}, 3 \mathrm{H}), 3.38(\mathrm{dd}, J=2.4,11.7 \mathrm{~Hz}$, $1 \mathrm{H}), 3.17(\mathrm{dd}, J=8.7,12.9 \mathrm{~Hz}, 1 \mathrm{H}), 2.88(\mathrm{dd}, J=4.2,15.9 \mathrm{~Hz}, 1 \mathrm{H}), 2.70(\mathrm{dd}, J=8.7,15.9$ $\mathrm{Hz}, 1 \mathrm{H}) . \mathrm{MS} m / z 409.3\left(\mathrm{M}+\mathrm{H}^{+}\right)$.

\section{$\mathrm{N}$-[6-Cyano-1-(3-methyl-3H-imidazol-4-ylmethyl)-1,2,3,4-tetrahydro-quinolin-3-yl]-4-fluoro- benzenesulfonamide (5)}

${ }^{1} \mathrm{H}$ NMR $\left(500 \mathrm{MHz}\right.$, methanol- $\left.d_{4}\right) \delta 8.35(\mathrm{~s}, 1 \mathrm{H}), 7.95-7.93(\mathrm{~m}, 2 \mathrm{H}), 7.46(\mathrm{~s}, 1 \mathrm{H}), 7.39(\mathrm{~d}, J$ $=8.5 \mathrm{~Hz}, 1 \mathrm{H}), 7.35(\mathrm{t}, J=8.0 \mathrm{~Hz}, 2 \mathrm{H}), 7.24(\mathrm{~s}, 1 \mathrm{H}), 6.82(\mathrm{~d}, J=8.5 \mathrm{~Hz}, 1 \mathrm{H}), 4.82(\mathrm{~d}, J=17.0$ $\mathrm{Hz}, 1 \mathrm{H}), 4.65(\mathrm{~d}, J=17.0 \mathrm{~Hz}, 1 \mathrm{H}), 3.95-3.85(\mathrm{~m}, 4 \mathrm{H}), 3.69-3.65(\mathrm{~m}, 1 \mathrm{H}), 3.55-3.45(\mathrm{~m}, 1 \mathrm{H})$, 2.97-2.96 (m, 1H), 2.71-2.61 (m, 1H). MS m/z $426.12\left(\mathrm{M}+\mathrm{H}^{+}\right)$.

\section{N-Alkylation}

To a suspension of $5(5 \mathrm{mmol})$ and $\mathrm{Cs}_{2} \mathrm{CO}_{3}(9.8 \mathrm{mmol})$ in dry DMF $(5 \mathrm{~mL})$ was added the appropriate alkyl halide $(5.4 \mathrm{mmol})$, and the mixture was stirred at room temperature overnight under argon. After addition of water $(20 \mathrm{~mL})$, the solution was extracted with ethyl acetate (3 $\times 20 \mathrm{~mL})$. The organic layer was extracted with brine $(3 \times 10 \mathrm{~mL})$. The combined organic layers were dried over $\mathrm{MgSO}_{4}$ and evaporated under reduce pressure. The residue was purified by HPLC. 
Pyridine-2-sulfonic Acid [6-Cyano-1-(3-methyl-3H-imidazol-4-ylmethyl)-1,2,3,4-tetrahydroquinolin-3-yl]-(4-methanesulfonyl-benzyl)-amide (69)

${ }^{1} \mathrm{H}$ NMR $\left(300 \mathrm{MHz}\right.$, methanol- $\left.d_{4}\right) \delta 8.86(\mathrm{~s}, 1 \mathrm{H}), 8.71(\mathrm{~d}, J=7.2 \mathrm{~Hz}, 1 \mathrm{H}), 8.10(\mathrm{td}, J=1.5$, $8.1 \mathrm{~Hz}, 1 \mathrm{H}), 7.99(\mathrm{dt}, J=1.2,8,1 \mathrm{~Hz}, 1 \mathrm{H}), 7.90(\mathrm{~d}, J=8.1 \mathrm{~Hz}, 2 \mathrm{H}), 7.69$ (ddd, $J=1.2,4.8$, $7.5 \mathrm{~Hz}, 1 \mathrm{H}), 7.61(\mathrm{~d}, J=8.1 \mathrm{~Hz}, 2 \mathrm{H}), 7.33(\mathrm{dd}, J=2.1,9.0 \mathrm{~Hz}, 1 \mathrm{H}), 7.28(\mathrm{~s}, 1 \mathrm{H}), 7.25(\mathrm{~d}, J=$ $2.1 \mathrm{~Hz}, 1 \mathrm{H}), 6.65(\mathrm{~d}, J=8.7 \mathrm{~Hz}, 1 \mathrm{H}), 4.79(\mathrm{~d}, J=17.1 \mathrm{~Hz}, 1 \mathrm{H}), 4.62(\mathrm{~d}, J=17.1 \mathrm{~Hz}, 1 \mathrm{H})$, 4.39-4.59 (m, 3H), $3.8(\mathrm{~s}, 3 \mathrm{H}), 3.55-3.62(\mathrm{~m}, 1 \mathrm{H}), 3.42-3.47(\mathrm{~m}, 1 \mathrm{H}), 3.15(\mathrm{~s}, 3 \mathrm{H}), 3.07-3.14$ (m, 1H), $2.97(\mathrm{dd}, J=4.5,15.9 \mathrm{~Hz}, 1 \mathrm{H}) . \mathrm{MS} m / z 577.4\left(\mathrm{M}+\mathrm{H}^{+}\right)$.

6-\{[[6-Cyano-1-(3-methyl-3H-imidazol-4-ylmethyl)-1,2,3,4-tetrahydro-quinolin-3-yl](pyridine-2-sulfonyl)-amino]-methyl\}-pyridine-2-carboxylic Acid Methyl Ester (67)

${ }^{1} \mathrm{H}$ NMR $\left(300 \mathrm{MHz}, \mathrm{CDCl}_{3}\right) \delta 8.78(\mathrm{~s}, 1 \mathrm{H}), 8.63(\mathrm{~d}, J=7.0 \mathrm{~Hz}, 1 \mathrm{H}), 7.78-8.04(\mathrm{~m}, 5 \mathrm{H}), 7.51-$ $7.76(\mathrm{~m}, 1 \mathrm{H}), 7.30-7.34(\mathrm{~m}, 2 \mathrm{H}), 7.18(\mathrm{~s}, 1 \mathrm{H}), 6.56(\mathrm{~d}, J=8.7 \mathrm{~Hz}, 1 \mathrm{H}), 4.76(\mathrm{~d}, J=17.1 \mathrm{~Hz}$, $1 \mathrm{H}), 4.65(\mathrm{~d}, J=17.1 \mathrm{~Hz}, 1 \mathrm{H}), 4.47-4.61(\mathrm{~m}, 3 \mathrm{H}), 3.96(\mathrm{~s}, 3 \mathrm{H}), 3.85(\mathrm{~s}, 3 \mathrm{H}), 3.54-3.62(\mathrm{~m}$, $1 \mathrm{H}), 3.42-3.51(\mathrm{~m}, 1 \mathrm{H}), 3.13(\mathrm{dd}, J=11.1,15 \mathrm{~Hz}, 1 \mathrm{H}), 2.91(\mathrm{dd}, J=3.6,15 \mathrm{~Hz}, 1 \mathrm{H}) . \mathrm{MS} m /$ z. $558.3\left(\mathrm{M}+\mathrm{H}^{+}\right)$.

4-\{[[6-Cyano-1-(3-methyl-3H-imidazol-4-ylmethyl)-1,2,3,4-tetrahydro-quinolin-3-yl](pyridine-2-sulfonyl)-amino]-methyl\}-piperidine-1-carboxylic Acid tert-Butyl Ester (6)

${ }^{1} \mathrm{H}$ NMR $\left(300 \mathrm{MHz}\right.$, methanol- $\left.d_{4}\right) \delta 8.93(\mathrm{~s}, 1 \mathrm{H}), 8.71(\mathrm{~d}, J=7.2 \mathrm{~Hz}, 1 \mathrm{H}), 8.05(\mathrm{td}, J=1.8$, $7.5 \mathrm{~Hz}, 1 \mathrm{H}), 8.01-8.02(\mathrm{~m}, 1 \mathrm{H}), 7.66(\mathrm{ddd}, J=1.2,4.8,7.5 \mathrm{~Hz}, 1 \mathrm{H}), 7.43(\mathrm{~s}, 1 \mathrm{H}), 7.39$ (dd, $J=1.5,8.4 \mathrm{~Hz}, 1 \mathrm{H}), 7.33(\mathrm{~s}, 1 \mathrm{H}), 6.80(\mathrm{~d}, J=9.7 \mathrm{~Hz}, 1 \mathrm{H}), 4.81(\mathrm{~d}, J=16.8 \mathrm{~Hz}, 1 \mathrm{H}), 4.68(\mathrm{~d}$, $J=16.8 \mathrm{~Hz}, 1 \mathrm{H}), 4.32-4.44(\mathrm{~m}, 1 \mathrm{H}), 4.05-4.09(\mathrm{~m}, 2 \mathrm{H}), 3.91(\mathrm{~s}, 3 \mathrm{H}), 3.58-3.66(\mathrm{~m}, 2 \mathrm{H})$, 3.53-3.55 (m, 1H), 3.11-3.21 (m, 3H), 2.87-2.88 (m, 1H), 2.63-2.69 (m, 1H), 1.64-1.77 (m, $3 \mathrm{H}), 1.3(\mathrm{~s}, 9 \mathrm{H}), 1.01-1.11(\mathrm{~m}, 2 \mathrm{H})$. MS $m / z 606.6\left(\mathrm{M}+\mathrm{H}^{+}\right)$.

Pyridine-2-sulfonic Acid [6-Cyano-1-(3-methyl-3H-imidazol-4-ylmethyl)-1,2,3,4-tetrahydroquinolin-3-yl]-(2-pyrazol-1-yl-ethyl)-amide (56)

${ }^{1} \mathrm{H}$ NMR $\left(300 \mathrm{MHz}\right.$, methanol- $\left.d_{4}\right) \delta 8.92(\mathrm{~s}, 1 \mathrm{H}), 8.73(\mathrm{~d}, J=6.9 \mathrm{~Hz}, 1 \mathrm{H}), 8.01-8.12(\mathrm{~m}, 2 \mathrm{H})$, $7.64-7.71(\mathrm{~m}, 1 \mathrm{H}), 7.61(\mathrm{~d}, J=2.1 \mathrm{~Hz}, 1 \mathrm{H}), 7.50(\mathrm{~d}, J=1.5 \mathrm{~Hz}, 1 \mathrm{H}), 7.32-7.38(\mathrm{~m}, 2 \mathrm{H}), 7.21$ $(\mathrm{s}, 1 \mathrm{H}), 6.79(\mathrm{~d}, J=8.7 \mathrm{~Hz}, 1 \mathrm{H}), 6.30(\mathrm{t}, J=2.1 \mathrm{~Hz}, 1 \mathrm{H}), 4.68(\mathrm{~d}, J=18.3 \mathrm{~Hz}, 1 \mathrm{H}), 4.60(\mathrm{~d}$, $J=18.3 \mathrm{~Hz}, 1 \mathrm{H}), 4.70(\mathrm{t}, J=6.3 \mathrm{~Hz}, 2 \mathrm{H}), 4.25-4.35(\mathrm{~m}, 1 \mathrm{H}), 3.83(\mathrm{~s}, 3 \mathrm{H}), 3.74(\mathrm{t}, J=6.3 \mathrm{~Hz}$, $2 \mathrm{H}), 3.10-3.21(\mathrm{~m}, 2 \mathrm{H}), 2.86(\mathrm{dd}, J=12,15.3 \mathrm{~Hz}, 1 \mathrm{H}), 2.58(\mathrm{dd}, J=3.9,15.3 \mathrm{~Hz}, 1 \mathrm{H}) . \mathrm{MS}$ $\mathrm{m} / \mathrm{z} 503.4\left(\mathrm{M}+\mathrm{H}^{+}\right)$.

\{3-[[6-Cyano-1-(3-methyl-3H-imidazol-4-ylmethyl)-1,2,3,4-tetrahydro-quinolin-3-yl](pyridine-2-sulfonyl)-amino]-propyl\}-methyl-carbamic Acid Methyl Ester (48)

${ }^{1} \mathrm{H}$ NMR $\left(300 \mathrm{MHz}\right.$, methanol- $\left.d_{4}\right) \delta 8.94(\mathrm{~d}, J=0.9 \mathrm{~Hz}, 1 \mathrm{H}), 8.70(\mathrm{~d}, J=7.5 \mathrm{~Hz}, 1 \mathrm{H}), 8.08$ $(\mathrm{td}, J=1.8,7.8 \mathrm{~Hz}, 1 \mathrm{H}), 8.0(\mathrm{dt}, J=1.2,7.8 \mathrm{~Hz}, 1 \mathrm{H}), 7.66(\mathrm{ddd}, J=1.5,4.8,7.5 \mathrm{~Hz}, 1 \mathrm{H}), 7.43$ $(\mathrm{d}, J=1.2 \mathrm{~Hz}, 1 \mathrm{H}), 7.38(\mathrm{dd}, J=2.1,8.7 \mathrm{~Hz}, 1 \mathrm{H}), 7.33(\mathrm{~s}, 1 \mathrm{H}), 6.81(\mathrm{~d}, J=8.7 \mathrm{~Hz}, 1 \mathrm{H}), 4.82$ $(\mathrm{d}, J=17.5 \mathrm{~Hz}, 1 \mathrm{H}), 4.62(\mathrm{~d}, J=17.5 \mathrm{~Hz}, 1 \mathrm{H}), 4.40-4.52(\mathrm{~m}, 1 \mathrm{H}), 3.92(\mathrm{~s}, 3 \mathrm{H}), 3.65(\mathrm{~s}, 3 \mathrm{H})$, 3.55-3.47 (m, 4H), 3.28-3.05 (m, 4H), 2.93 (s, 3H), 1.78-1.88 (m, 2H). MS m/z $538.4(\mathrm{M}+$ $\left.\mathrm{H}^{+}\right)$.

Pyridine-2-sulfonic Acid [6-Cyano-1-(3-methyl-3H-imidazol-4-ylmethyl)-1,2,3,4-tetrahydroquinolin-3-yl]-(4-fluoro-benzyl)-amide (71)

${ }^{1} \mathrm{H}$ NMR $\left(300 \mathrm{MHz}\right.$, methanol- $\left.d_{4}\right) \delta 8.89(\mathrm{~s}, 1 \mathrm{H}), 8.74(\mathrm{~d}, J=6.5 \mathrm{~Hz}, 1 \mathrm{H}), 8.09(\mathrm{td}, J=1.8$, $7.8 \mathrm{~Hz}, 1 \mathrm{H}), 8.0(\mathrm{dt}, J=1.2,7.8 \mathrm{~Hz}, 1 \mathrm{H}), 7.67$ (ddd, $J=1.5,4.8,7.5 \mathrm{~Hz}, 1 \mathrm{H}), 7.39-7.43(\mathrm{~m}$, $2 \mathrm{H}), 7.35(\mathrm{dd}, J=2.1,8.7 \mathrm{~Hz}, 1 \mathrm{H}), 7.33(\mathrm{~s}, 1 \mathrm{H}), 7.25(\mathrm{~s}, 1 \mathrm{H}), 7.01-7.07(\mathrm{~m}, 2 \mathrm{H}), 6.75(\mathrm{~d}, J=$ 
8.7 Hz, 1H), 4.43-4.69 (m, 5H), 3.82 (s, 3H), 3.55-3.47 (m, 1H), 3.17-3.25 (m, 1H), 3.07 (dd, $J=12,15 \mathrm{~Hz}, 1 \mathrm{H}), 2.91(\mathrm{dd}, J=3.9,15 \mathrm{~Hz}, 1 \mathrm{H}) . \mathrm{MS} m / z 517.4\left(\mathrm{M}+\mathrm{H}^{+}\right)$.

Pyridine-2-sulfonic Acid [6-Cyano-1-(3-methyl-3H-imidazol-4-ylmethyl)-1,2,3,4-tetrahydroquinolin-3-yl]-[2-(2-trifluoromethyl-phenyl)-ethyl]-amide (76)

${ }^{1} \mathrm{H}$ NMR $\left(300 \mathrm{MHz}\right.$, methanol- $\left.d_{4}\right) \delta 8.87(\mathrm{~s}, 1 \mathrm{H}), 8.74(\mathrm{~d}, J=4.5 \mathrm{~Hz}, 1 \mathrm{H}), 8.12(\mathrm{td}, J=1.8$, $7.8 \mathrm{~Hz}, 1 \mathrm{H}), 8.0(\mathrm{dt}, J=1.2,7.8 \mathrm{~Hz}, 1 \mathrm{H}), 7.63-7.71(\mathrm{~m}, 2 \mathrm{H}), 7.58-7.63(\mathrm{~m}, 1 \mathrm{H}), 7.37-7.49$ $(\mathrm{m}, 5 \mathrm{H}), 6.81(\mathrm{~d}, J=8.7 \mathrm{~Hz}, 1 \mathrm{H}), 4.92(\mathrm{~d}, J=17.7 \mathrm{~Hz}, 1 \mathrm{H}), 4.82(\mathrm{~d}, J=17.7 \mathrm{~Hz}, 1 \mathrm{H}), 4.50-$ $4.56(\mathrm{~m}, 1 \mathrm{H}), 3.98(\mathrm{~s}, 3 \mathrm{H}), 3.55-3.47(\mathrm{~m}, 4 \mathrm{H}), 3.18-3.31(\mathrm{~m}, 3 \mathrm{H}), 3.02-3.08(\mathrm{~m}, 1 \mathrm{H}) . \mathrm{MS} \mathrm{m} /$ z $581.4\left(\mathrm{M}+\mathrm{H}^{+}\right)$.

1-Methyl-1H-imidazole-4-sulfonic Acid [6-Cyano-1-(3-methyl-3H-imidazol-4ylmethyl)-1,2,3,4-tetrahydro-quinolin-3-yl]-[2-(2-fluoro-phenyl)-ethyl]-amide (113)

${ }^{1} \mathrm{H}$ NMR $\left(300 \mathrm{MHz}\right.$, methanol- $\left.d_{4}\right) \delta 8.93(\mathrm{~s}, 1 \mathrm{H}), 7.76(\mathrm{~s}, 1 \mathrm{H}), 7.73(\mathrm{~s}, 1 \mathrm{H}), 7.51(\mathrm{~s}, 1 \mathrm{H}), 7.38$ $(\mathrm{dd}, J=2.1,8.7 \mathrm{~Hz}, 1 \mathrm{H}), 7.23-7.32(\mathrm{~m}, 3 \mathrm{H}), 7.10-7.17(\mathrm{~m}, 2 \mathrm{H}), 6.84(\mathrm{~d}, J=8.6 \mathrm{~Hz}, 1 \mathrm{H}), 4.92$ $(\mathrm{d}, J=17.7 \mathrm{~Hz}, 1 \mathrm{H}), 4.82(\mathrm{~d}, J=17.7 \mathrm{~Hz}, 1 \mathrm{H}), 4.48-4.50(\mathrm{~m}, 1 \mathrm{H}), 4.07(\mathrm{~s}, 3 \mathrm{H}), 3.82(\mathrm{~s}, 3 \mathrm{H})$, 3.35-3.57 (m, 4H), 3.16-3.29 (m, 3H), 2.92-3.00 (m, 1H). MS m/z $534.4\left(\mathrm{M}+\mathrm{H}^{+}\right)$.

1-Methyl-1 $\mathrm{H}$-imidazole-4-sulfonic Acid [6-Cyano-1-(3-methyl-3 $\mathrm{H}$-imidazol-4ylmethyl)-1,2,3,4-tetrahydro-quinolin-3-yl]-[2-(4-fluoro-phenyl)-ethyl]-amide (112)

${ }^{1} \mathrm{H}$ NMR (300 MHz, acetone- $d_{6}$ ) $\delta 8.93(\mathrm{~s}, 1 \mathrm{H}), 7.75(\mathrm{~s}, 1 \mathrm{H}), 7.74(\mathrm{~s}, 1 \mathrm{H}), 7.49(\mathrm{~s}, 1 \mathrm{H}), 7.38$ (dd, $J=2.1,8.7 \mathrm{~Hz}, 1 \mathrm{H}), 7.32(\mathrm{~s}, 1 \mathrm{H}) 7.22-7.25(\mathrm{~m}, 2 \mathrm{H}), 7.01-7.07(\mathrm{~m}, 2 \mathrm{H}), 6.93(\mathrm{~d}, J=8.6$ $\mathrm{Hz}, 1 \mathrm{H}), 4.93(\mathrm{~d}, J=16.8 \mathrm{~Hz}, 1 \mathrm{H}), 4.80(\mathrm{~d}, J=16.8 \mathrm{~Hz}, 1 \mathrm{H}), 4.38-4.46(\mathrm{~m}, 1 \mathrm{H}), 4.07(\mathrm{~s}, 3 \mathrm{H})$, $3.82(\mathrm{~s}, 3 \mathrm{H}), 3.34-3.54(\mathrm{~m}, 4 \mathrm{H}), 3.07(\mathrm{dd}, J=12,15 \mathrm{~Hz}, 1 \mathrm{H}), 2.98(\mathrm{t}, J=8.1 \mathrm{~Hz}, 2 \mathrm{H}) 2.90$ (dd, $J=3.7,15.2 \mathrm{~Hz}, 1 \mathrm{H})$. MS $m / z 534.4\left(\mathrm{M}+\mathrm{H}^{+}\right)$.

1-Methyl-1 $\mathrm{H}$-imidazole-4-sulfonic Acid [6-Cyano-1-(3-methyl-3H-imidazol-4ylmethyl)-1,2,3,4-tetrahydro-quinolin-3-yl]-(4-fluoro-benzyl)-amide (109)

${ }^{1} \mathrm{H}$ NMR (300 MHz, methanol- $\left.d_{4}\right) \delta 8.90(\mathrm{~s}, 1 \mathrm{H}), 7.81(\mathrm{~s}, 1 \mathrm{H}), 7.73(\mathrm{~s}, 1 \mathrm{H}), 7.36-7.42(\mathrm{~m}$, $3 \mathrm{H}), 7.33(\mathrm{~s}, 1 \mathrm{H}), 7.27(\mathrm{~s}, 1 \mathrm{H}), 7.10-7.17(\mathrm{~m}, 2 \mathrm{H}), 6.84(\mathrm{~d}, J=8.6 \mathrm{~Hz}, 1 \mathrm{H}), 4.28-4.65(\mathrm{~m}$, $5 \mathrm{H}), 3.87$ (s, 3H), 3.80 (s, 3H), 3.34-3.39 (m, 1H), 3.18-3.26 (m, 1H), 3.07 (dd, $J=11.4,15.3$ $\mathrm{Hz}, 1 \mathrm{H}), 2.87(\mathrm{dd}, J=3.7,15.2 \mathrm{~Hz}, 1 \mathrm{H}) . \mathrm{MS} m / z .520 .4\left(\mathrm{M}+\mathrm{H}^{+}\right)$.

\section{1-Methyl-1 H-imidazole-4-sulfonic Acid [6-Cyano-1-(3-methyl-3H-imidazol-4- ylmethyl)-1,2,3,4-tetrahydro-quinolin-3-yl]-(4-ethanesulfonyl-benzyl)-amide (132)}

${ }^{1} \mathrm{H}$ NMR $\left(300 \mathrm{MHz}\right.$, methanol- $\left.d_{4}\right) \delta 8.87(\mathrm{~s}, 1 \mathrm{H}), 7.87(\mathrm{~d}, J=8.1 \mathrm{~Hz}, 2 \mathrm{H}), 7.85(\mathrm{~s}, 1 \mathrm{H}), 7.77$ (s, $1 \mathrm{H}), 7.62(\mathrm{~d}, J) 8.1 \mathrm{~Hz}, 2 \mathrm{H}), 7.34(\mathrm{dd}, J=1.8,8.7 \mathrm{~Hz}, 1 \mathrm{H}), 7.25(\mathrm{~d}, J=1.8 \mathrm{~Hz}, 1 \mathrm{H}), 6.68$ $(\mathrm{d}, J=8.7 \mathrm{~Hz}), 4.75(\mathrm{~d}, 2 \mathrm{H}), 4.53-4.55(\mathrm{~m}, 1 \mathrm{H}), 4.51(\mathrm{~d}, J=17.7 \mathrm{~Hz}, 1 \mathrm{H}), 4.45(\mathrm{~d}, J=17.7$ $\mathrm{Hz}, 1 \mathrm{H}), 3.81$ (s, 3H), 3.80 (s, 3H), 3.10-3.25 (m, 6H), 2.95-3.05 (m, 1H), 1.21-1.25 (t, 3H). $\mathrm{MS} m / z 594.5\left(\mathrm{M}+\mathrm{H}^{+}\right)$.

1-Methyl-1 H-imidazole-4-sulfonic Acid (4-Benzenesulfonyl-benzyl)-[6-cyano-1-(3-methyl-3Himidazol-4-ylmethyl)-1,2,3,4-tetrahydro-quinolin-3-yl]-amide (145)

${ }^{1} \mathrm{H}$ NMR (300 MHz, methanol- $\left.d_{4}\right) \delta 8.89(\mathrm{~s}, 1 \mathrm{H}), 7.89-8.10(\mathrm{~m}, 4 \mathrm{H}), 7.87(\mathrm{~d}, J=8.1 \mathrm{~Hz}, 2 \mathrm{H})$, $7.77(\mathrm{~s}, 1 \mathrm{H}), 7.50-7.72(\mathrm{~m}, 5 \mathrm{H}), 7.34(\mathrm{dd}, J=1.8,8.7 \mathrm{~Hz}, 1 \mathrm{H}), 7.25(\mathrm{~d}, J=1.8 \mathrm{~Hz}, 1 \mathrm{H}), 6.68$ $(\mathrm{d}, J=8.7 \mathrm{~Hz}, 1 \mathrm{H}), 4.75(\mathrm{~m}, 2 \mathrm{H}), 4.53-4.55(\mathrm{~m}, 1 \mathrm{H}), 4.51(\mathrm{~d}, J=17.7 \mathrm{~Hz}, 1 \mathrm{H}), 4.45(\mathrm{~d}, J=$ $17.7 \mathrm{~Hz}, 1 \mathrm{H}), 3.81$ (s, 3H), 3.80 (s, 3H), 3.40-3.45 (m, 2H), 2.95-3.05 (m, 1H). MS m/z 642.6 $\left(\mathrm{M}+\mathrm{H}^{+}\right)$. 
1-Methyl-1 H-imidazole-4-sulfonic Acid [6-Cyano-1-(3-methyl-3H-imidazol-4-

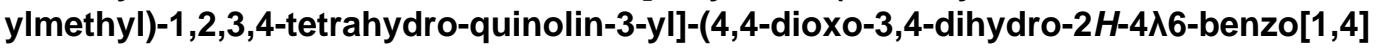
oxathiin-7-ylmethyl)-amide (146)

${ }^{1} \mathrm{H}$ NMR $\left(300 \mathrm{MHz}\right.$, methanol- $\left.d_{4}\right) \delta 8.90(\mathrm{~s}, 1 \mathrm{H}), 7.85(\mathrm{~s}, 1 \mathrm{H}), 7.68(\mathrm{~d}, J=8.1 \mathrm{~Hz}, 1 \mathrm{H}), 7.45$ $(\mathrm{s}, 1 \mathrm{H}), 7.30(\mathrm{~s}, 1 \mathrm{H}), 7.28(\mathrm{dd}, J=1.8,8.7 \mathrm{~Hz}, 1 \mathrm{H}), 7.25(\mathrm{~d}, J=1.8 \mathrm{~Hz}, 1 \mathrm{H}), 7.15(\mathrm{dd}, J=8.7$, $1.8 \mathrm{~Hz}, 1 \mathrm{H}), 7.08(\mathrm{~d}, J=2.1 \mathrm{~Hz}, 1 \mathrm{H}), 6.68(\mathrm{~d}, J=8.7 \mathrm{~Hz}, 1 \mathrm{H}), 4.48-4.52(\mathrm{~m}, 2 \mathrm{H}), 4.51(\mathrm{~d}, J$ $=17.7 \mathrm{~Hz}, 1 \mathrm{H}), 4.45(\mathrm{~d}, J=17.7 \mathrm{~Hz}, 1 \mathrm{H}), 3.90(\mathrm{~s}, 3 \mathrm{H}), 3.80(\mathrm{~s}, 3 \mathrm{H}), 3.55-3.60(\mathrm{~m}, 2 \mathrm{H}), 3.40-$ $3.55(\mathrm{~m}, 2 \mathrm{H}), 2.95-3.05(\mathrm{~m}, 1 \mathrm{H}), 2.68-2.75(\mathrm{~m}, 1 \mathrm{H}) . \mathrm{MS} \mathrm{m} / \mathrm{z} 608.4\left(\mathrm{M}+\mathrm{H}^{+}\right)$.

4-\{[[6-Cyano-1-(3-methyl-3H-imidazol-4-ylmethyl)-1,2,3,4-tetrahydro-quinolin-3-yl]-(1,5dimethyl-1 $\mathrm{H}$-imidazole-4-sulfonyl)-amino]-methyl\}-piperidine-1-carboxylic Acid Methyl Ester (234)

${ }^{1} \mathrm{H}$ NMR $\left(300 \mathrm{MHz}\right.$, methanol- $\left.d_{4}\right) \delta 8.93(\mathrm{~s}, 1 \mathrm{H}), 7.73(\mathrm{~s}, 1 \mathrm{H}), 7.40(\mathrm{~s}, 1 \mathrm{H}), 7.37(\mathrm{dd}, J=3.0$, $9.0 \mathrm{~Hz}, 1 \mathrm{H}), 7.32(\mathrm{~d}, J=1.8 \mathrm{~Hz}, 1 \mathrm{H}), 6.79(\mathrm{~d}, J) 9 \mathrm{~Hz}, 1 \mathrm{H}), 4.81(\mathrm{~d}, J=18.0 \mathrm{~Hz}, 1 \mathrm{H}), 4.68(\mathrm{~d}$, $J=18.0 \mathrm{~Hz}, 1 \mathrm{H}), 4.32-4.42(\mathrm{~m}, 1 \mathrm{H}), 4.08-4.18(\mathrm{~m}, 2 \mathrm{H}), 3.92(\mathrm{~s}, 3 \mathrm{H}), 3.67(\mathrm{~s}, 3 \mathrm{H}), 3.68(\mathrm{~s}$, $3 \mathrm{H}), 3.51-3.58(\mathrm{~m}, 2 \mathrm{H}), 2.98-3.17(\mathrm{~m}, 3 \mathrm{H}), 2.73-2.87(\mathrm{~m}, 3 \mathrm{H}), 2.48(\mathrm{~s}, 3 \mathrm{H}), 1.69-1.85$, (m, $3 \mathrm{H}), 1.01-1.27(\mathrm{~m}, 2 \mathrm{H}) . \mathrm{MS} \mathrm{m} / z 581.6\left(\mathrm{M}+\mathrm{H}^{+}\right)$.

4-\{[[6-Cyano-1-(3-methyl-3H-imidazol-4-ylmethyl)-1,2,3,4-tetrahydro-quinolin-3yl]-(1,5dimethyl-1H-imidazole-4-sulfonyl)-amino]-methyl\}-piperidine-1-carboxylic Acid tert-Butyl Ester (235)

${ }^{1} \mathrm{H}$ NMR $\left(300 \mathrm{MHz}\right.$, methanol- $\left.d_{4}\right) \delta 8.93(\mathrm{~s}, 1 \mathrm{H}), 7.73(\mathrm{~s}, 1 \mathrm{H}), 7.43(\mathrm{~d}, J=1.8 \mathrm{~Hz}, 1 \mathrm{H}), 7.37$ (dd, $J=3,9 \mathrm{~Hz}, 1 \mathrm{H}), 7.32(\mathrm{~s}, 1 \mathrm{H}), 6.79(\mathrm{~s}, 1 \mathrm{H}), 4.81(\mathrm{~d}, J=18.0 \mathrm{~Hz}, 1 \mathrm{H}), 4.68(\mathrm{~d}, J=18.0$ $\mathrm{Hz}, 1 \mathrm{H}), 4.33-4.40(\mathrm{~m}, 1 \mathrm{H}), 4.06-4.11(\mathrm{~m}, 2 \mathrm{H}), 3.92(\mathrm{~s}, 3 \mathrm{H}), 3.67(\mathrm{~s}, 3 \mathrm{H}), 3.53-3.58(\mathrm{~m}, 2 \mathrm{H})$, 3.09-3.17 (m, 3 H), 2.64-2.81 (m, 3H), 2.48 (s, 3H), 1.63-1.81 (m, 3H), 1.4 (s, 9 H), 0.90$1.13(\mathrm{~m}, 2 \mathrm{H}) . \mathrm{MS} m / 2623.6\left(\mathrm{M}+\mathrm{H}^{+}\right)$.

1-Methyl-1 H-imidazole-4-sulfonic Acid [6-Cyano-1-(3-methyl-3H-imidazol-4ylmethyl)-1,2,3,4-tetrahydro-quinolin-3-yl]-[2-(2-oxo-pyrrolidin-1-yl)-ethyl]-amide (117)

${ }^{1} \mathrm{H}$ NMR $\left(300 \mathrm{MHz}\right.$, methanol- $\left.d_{4}\right) \delta 8.91(\mathrm{~s}, 1 \mathrm{H}), 7.30-7.45(\mathrm{~m}, 2 \mathrm{H}), 7.17-7.24(\mathrm{~m}, 1 \mathrm{H}), 7.12$ $(\mathrm{s}, 1 \mathrm{H}), 6.82(\mathrm{~d}, J=9.0 \mathrm{~Hz}, 1 \mathrm{H}), 6.52(\mathrm{~d}, J=9.0 \mathrm{~Hz}, 1 \mathrm{H}), 4.28-4.40(\mathrm{~m}, 2 \mathrm{H}), 3.93(\mathrm{~s}, 3 \mathrm{H})$, $3.80(\mathrm{~s}, 3 \mathrm{H}), 3.66-3.73(\mathrm{~m}, 1 \mathrm{H}), 3.51-3.63(\mathrm{~m}, 2 \mathrm{H}), 3.40-3.48(\mathrm{~m}, 2 \mathrm{H}), 3.03-3.21(\mathrm{~m}, 2 \mathrm{H})$, 2.78-2.93 (m, 2H), 2.62-2.76 (m, 2H), $2.38(\mathrm{t}, J=6.0 \mathrm{~Hz}, 2 \mathrm{H}), 2.07(\mathrm{t}, J=6.0 \mathrm{~Hz}, 2 \mathrm{H}) . \mathrm{MS}$ $\mathrm{m} / z 523.3\left(\mathrm{M}+\mathrm{H}^{+}\right)$.

1,5-Dimethyl-1 H-imidazole-4-sulfonic Acid [6-Cyano-1-(3-methyl-3H-imidazol-4ylmethyl)-1,2,3,4-tetrahydro-quinolin-3-yl]-(4-methanesulfonyl-benzyl)-amide (236)

${ }^{1} \mathrm{H}$ NMR $\left(300 \mathrm{MHz}\right.$, methanol- $\left.d_{4}\right) \delta 8.85(\mathrm{~s} 1 \mathrm{H}), 7.91(\mathrm{~d}, J=9.0 \mathrm{~Hz}, 2 \mathrm{H}), 7.77(\mathrm{~s}, 1 \mathrm{H}), 7.60$ (d, $J=9.0 \mathrm{~Hz}, 2 \mathrm{H}), 7.35(\mathrm{~d}, J=9.0 \mathrm{~Hz} 2 \mathrm{H}), 7.29(\mathrm{~s}, 1 \mathrm{H}), 6.68(\mathrm{~d}, J=9.0 \mathrm{~Hz}, 1 \mathrm{H}), 4.40-4.71$ (m, 5H), 3.83 (s, 3H), 3.68 (s, 3H), 3.45-3.55 (m, 2H), 3.17 (s, 3H), 2.88-3.02 (m, 2H), 2.42 (s, 3H). MS m/z $594.5\left(\mathrm{M}+\mathrm{H}^{+}\right)$.

1,5-Dimethyl-1H-imidazole-4-sulfonic Acid [6-Cyano-1-(3-methyl-3H-imidazol-4ylmethyl)-1,2,3,4-tetrahydro-quinolin-3-yl]-(4-ethanesulfonyl-benzyl)-amide (237)

${ }^{1} \mathrm{H}$ NMR $\left(300 \mathrm{MHz}\right.$, methanol- $\left.d_{4}\right) \delta 8.88(\mathrm{~s} 1 \mathrm{H}), 7.85(\mathrm{~d}, J=9.0 \mathrm{~Hz}, 2 \mathrm{H}), 7.78(\mathrm{~s}, 1 \mathrm{H}), 7.60$ $(\mathrm{d}, J=9.0 \mathrm{~Hz}, 2 \mathrm{H}), 7.35(\mathrm{~d}, J=9.0 \mathrm{~Hz} 2 \mathrm{H}), 7.29(\mathrm{~s}, 1 \mathrm{H}), 6.68(\mathrm{~d}, J=9.0 \mathrm{~Hz}, 1 \mathrm{H}), 4.40-4.76$ $(\mathrm{m}, 5 \mathrm{H}), 3.97(\mathrm{~s}, 3 \mathrm{H}), 3.82(\mathrm{~s}, 3 \mathrm{H}), 3.45-3.55(\mathrm{~m}, 2 \mathrm{H}), 3.15-3.30(\mathrm{~m}, 2 \mathrm{H}), 2.88-3.05(\mathrm{~m}, 2 \mathrm{H})$, $2.48(\mathrm{~s}, 3 \mathrm{H}), 1.22(\mathrm{t}, J=9.6 \mathrm{~Hz}, 3 \mathrm{H}) . \mathrm{MS} m / z 608.4\left(\mathrm{M}+\mathrm{H}^{+}\right)$. 


\title{
1,5-Dimethyl-1 H-imidazole-4-sulfonic Acid (5-Bromo-2-fluorobenzyl)-[6-cyano-1-(3- methyl-3H-imidazol-4-ylmethyl)-1,2,3,4-tetrahydro-quinolin-3-yl]-amide (238)
}

${ }^{1} \mathrm{H}$ NMR (300 MHz, methanol- $\left.d_{4}\right) \delta 8.92(\mathrm{~s}, 1 \mathrm{H}), 7.76(\mathrm{~s}, 1 \mathrm{H}), 7.53(\mathrm{~m}, 1 \mathrm{H}), 7.31-7.45(\mathrm{~m}$, $3 \mathrm{H}), 7.28(\mathrm{~s}, 1 \mathrm{H}), 6.99(\mathrm{~d}, J=9.0 \mathrm{~Hz}, 1 \mathrm{H}), 6.72(\mathrm{~d}, J=9.0 \mathrm{~Hz}, 1 \mathrm{H}), 4.42-4.75(\mathrm{~m}, 5 \mathrm{H}), 3.89$ (s, 3H), 3.67 (s, 3H), 3.50-3.62 (m, 2H), 2.95-3.10 (m, 2H), 2.40 (s, 3H). MS m/z $612.8(\mathrm{M}+$ $\left.\mathrm{H}^{+}\right)$.

\author{
1-Methyl-1 H-imidazole-4-sulfonic Acid [6-Cyano-1-(3-methyl-3H-imidazol-4- \\ ylmethyl)-1,2,3,4-tetrahydro-quinolin-3-yl]-(4-methanesulfonyl-benzyl)-amide (131) \\ ${ }^{1} \mathrm{H}$ NMR (300 MHz, methanol- $\left.d_{4}\right) \delta 9.0(\mathrm{~s} 1 \mathrm{H}), 7.95-7.85(\mathrm{~m}, 3 \mathrm{H}), 7.60(\mathrm{~d}, J=8.7 \mathrm{~Hz}, 2 \mathrm{H})$, \\ $7.35(\mathrm{t}, J=7.8 \mathrm{~Hz}, 3 \mathrm{H}), 7.29(\mathrm{~s}, 1 \mathrm{H}), 6.70(\mathrm{~d}, J=8.7 \mathrm{~Hz}, 1 \mathrm{H}), 4.60-4.40(\mathrm{~m}, 4 \mathrm{H}), 4.40-4.30$ \\ $(\mathrm{m}, 1 \mathrm{H}), 3.85(\mathrm{~s}, 3 \mathrm{H}), 3.80(\mathrm{~s}, 3 \mathrm{H}), 3.40-3.30(\mathrm{~m}, 2 \mathrm{H}), 3.0-2.90(\mathrm{~m}, 1 \mathrm{H}), 2.83-2.75(\mathrm{~m}, 1 \mathrm{H})$, \\ $2.50(\mathrm{~s}, 3 \mathrm{H}) . \mathrm{MS} m / z, 580.5\left(\mathrm{M}+\mathrm{H}^{+}\right)$.
}

1-Methyl-1H-imidazole-4-sulfonic Acid [6-Cyano-1-(3-methyl-3H-imidazol-4-ylmethyl)-1,2,3,4-tetrahydro-quinolin-3-yl]-(3-methoxy-propyl)-amide (91)

${ }^{1} \mathrm{H}$ NMR $\left(300 \mathrm{MHz}\right.$, methanol- $\left.d_{4}\right) \delta 8.92(\mathrm{~s}, 1 \mathrm{H}), 7.80(\mathrm{~s}, 1 \mathrm{H}), 7.75(\mathrm{~s}, 1 \mathrm{H}), 7.43(\mathrm{~s}, 1 \mathrm{H}), 7.39$ $(\mathrm{d}, J=8.7 \mathrm{~Hz}, 1 \mathrm{H}), 7.32(\mathrm{~s}, 1 \mathrm{H}), 6.81(\mathrm{~d}, J=8.7 \mathrm{~Hz}, 1 \mathrm{H}), 4.78(\mathrm{~d}, J=16.7 \mathrm{~Hz}, 1 \mathrm{H}), 4.69$ (d, $J=16.7 \mathrm{~Hz}, 1 \mathrm{H}), 4.45-4.38(\mathrm{~m}, 1 \mathrm{H}), 3.92(\mathrm{~s}, 3 \mathrm{H}), 3.81(\mathrm{~s}, 3 \mathrm{H}), 3.59-3.51(\mathrm{~m}, 2 \mathrm{H}), 3.43-3.38$ $(\mathrm{m}, 2 \mathrm{H}), 3.32(\mathrm{~s}, 3 \mathrm{H}), 3.28-3.20(\mathrm{~m}, 2 \mathrm{H}), 3.12-3.07(\mathrm{~m}, 1 \mathrm{H}), 2.85(\mathrm{dd}, J=3.3,15.3 \mathrm{~Hz}, 1 \mathrm{H})$, 1.96-1.85 (m, 4H). MS $m / z 484.5\left(\mathrm{M}+\mathrm{H}^{+}\right)$.

1-Methyl-1 H-imidazole-4-sulfonic Acid [6-Cyano-1-(3-methyl-3H-imidazol-4ylmethyl)-1,2,3,4-tetrahydro-quinolin-3-yl]-(5-trifluoromethyl-furan-2-ylmethyl)-amide (108)

${ }^{1} \mathrm{H}$ NMR (300 MHz, methanol-d $\left.d_{4}\right) \delta 8.90(\mathrm{~s}, 1 \mathrm{H}), 7.72(\mathrm{~s}, 1 \mathrm{H}), 7.60(\mathrm{~s}, 1 \mathrm{H}), 7.42-7.36(\mathrm{~m}$, 2H), $7.40(\mathrm{~s}, 1 \mathrm{H}), 6.90(\mathrm{~s}, 1 \mathrm{H}), 6.70(\mathrm{~d}, J=8.5 \mathrm{~Hz}, 1 \mathrm{H}), 6.50(\mathrm{~s}, 1 \mathrm{H}), 4.75-4.45(\mathrm{~m}, 5 \mathrm{H}), 3.90$ $(\mathrm{s}, 3 \mathrm{H}), 3.75(\mathrm{~s}, 3 \mathrm{H}), 3.50-3.40(\mathrm{~m}, 2 \mathrm{H}), 3.20-3.12(\mathrm{~m}, 1 \mathrm{H}), 2.89(\mathrm{dd}, J=3.2,15.1 \mathrm{~Hz}, 1 \mathrm{H})$. $\mathrm{MS} m / z 560.4\left(\mathrm{M}+\mathrm{H}^{+}\right)$.

\section{1-Methyl-1H-imidazole-4-sulfonic Acid [6-Cyano-1-(3-methyl-3H-imidazol-4- ylmethyl)-1,2,3,4-tetrahydro-quinolin-3-yl]-[4-(1,3-dioxo-1,3-dihydro-isoindol-2-yloxy)- butyl]-amide (143)}

${ }^{1} \mathrm{H}$ NMR $\left(300 \mathrm{MHz}\right.$, methanol- $\left.d_{4}\right) \delta 8.90(\mathrm{~s} 1 \mathrm{H}), 7.78-7.65(\mathrm{~m}, 6 \mathrm{H}), 7.60(\mathrm{~s}, 1 \mathrm{H}), 7.40-7.30$ $(\mathrm{m}, 2 \mathrm{H}), 6.80(\mathrm{~d}, J=8.7 \mathrm{~Hz}, 1 \mathrm{H}), 4.85(\mathrm{~d}, J=16.7 \mathrm{~Hz}, 1 \mathrm{H}), 4.75(\mathrm{~d}, J=16.7 \mathrm{~Hz}, 1 \mathrm{H}), 4.50-$ $4.40(\mathrm{~m}, 1 \mathrm{H}), 4.20(\mathrm{t}, 2 \mathrm{H}), 4.0(\mathrm{~s}, 3 \mathrm{H}), 3.80(\mathrm{~s}, 3 \mathrm{H}), 3.70-3.55(\mathrm{~m}, 2 \mathrm{H}), 3.40-3.20(\mathrm{~m}, 3 \mathrm{H})$, $2.89(\mathrm{dd}, J=3.2,15.1 \mathrm{~Hz}, 1 \mathrm{H}), 1.96-1.70(\mathrm{~m}, 4 \mathrm{H}) . \mathrm{MS}(\mathrm{EI}) \mathrm{m} / z 629.6\left(\mathrm{M}+\mathrm{H}^{+}\right)$.

\section{General Procedure for the Synthesis of Compounds According to Scheme 2}

Compound 6 (400 mg) was dissolved in dichloromethane $(5 \mathrm{~mL})$, and trifluoroacetic acid (1 $\mathrm{mL}$ ) was added. The reaction mixture was stirred for $45 \mathrm{~min}$. Solvent was removed in vacuo with care to be sure that all trifluoroacetic acid was removed. The residue was used without further purification.

The residue $(0.28 \mathrm{mmol})$ was dissolved in dichloromethane $(2.5 \mathrm{~mL})$ together with diisopropylethyl amine $(0.33 \mathrm{mmol})$. To this solution was added the appropriate chloroformate, isocyanate, or sulfonyl chloride $(0.30 \mathrm{mmol})$ dropwise at $0{ }^{\circ} \mathrm{C}$, and the mixture was stirred for $1 \mathrm{~h}$. Aqueous $\mathrm{NH}_{4} \mathrm{OH}(1 \mathrm{~mL})$ was added to the reaction mixture. After being stirred for an additional $15 \mathrm{~min}$, the mixture was diluted with ethyl acetate $(50 \mathrm{~mL})$ and washed with brine. The organic layer was dried over $\mathrm{MgSO}_{4}$ and evaporated under reduced pressure to yield the crude product, which was purified by preparative HPLC. The appropriate fractions were 
combined and concentrated in vacuo to give the corresponding derivatives as the trifluoroacetate salt.

Pyridine-2-sulfonic Acid [6-Cyano-1-(3-methyl-3H-imidazol-4-yImethyl)-1,2,3,4-tetrahydroquinolin-3-yl]-piperidin-4-ylmethyl-amide (54)

${ }^{1} \mathrm{H}$ NMR $\left(300 \mathrm{MHz}\right.$, methanol- $\left.d_{4}\right) \delta 8.93(\mathrm{~s}, 1 \mathrm{H}), 8.71(\mathrm{~d}, J=7.2 \mathrm{~Hz}, 1 \mathrm{H}), 8.07(\mathrm{td}, J=1.5$, $7.5 \mathrm{~Hz}, 1 \mathrm{H}), 8.01(\mathrm{dt}, J=0.9,7.8 \mathrm{~Hz}, 1 \mathrm{H}), 7.66(\mathrm{ddd}, J=1.5,4.8,7.5 \mathrm{~Hz}, 1 \mathrm{H}), 7.40(\mathrm{~s}, 1 \mathrm{H})$, $7.37(\mathrm{~d}, J=2.1 \mathrm{~Hz}, 1 \mathrm{H}), 7.33(\mathrm{~s}, 1 \mathrm{H}), 6.79(\mathrm{~d}, J=8.7 \mathrm{~Hz}, 1 \mathrm{H}), 4.80(\mathrm{~d}, J=17.4 \mathrm{~Hz}, 1 \mathrm{H}), 4.65$ (d, $J=17.4 \mathrm{~Hz}, 1 \mathrm{H}), 4.33-4.44(\mathrm{~m}, 1 \mathrm{H}), 3.91(\mathrm{~s}, 3 \mathrm{H}), 3.63(\mathrm{t}, J=10.8 \mathrm{~Hz}, 1 \mathrm{H}), 3.40-3.54(\mathrm{~m}$, $4 \mathrm{H}), 3.14-3.23(\mathrm{~m}, 2 \mathrm{H}), 2.82-3.02(\mathrm{~m}, 3 \mathrm{H}), 1.97-2.09(\mathrm{~m}, 3 \mathrm{H}), 1.30-1.52(\mathrm{~m}, 2 \mathrm{H}) . \mathrm{MS} \mathrm{m} / \mathrm{z}$ $506.5\left(\mathrm{M}+\mathrm{H}^{+}\right)$.

4-\{[[6-Cyano-1-(3-methyl-3H-imidazol-4-ylmethyl)-1,2,3,4-tetrahydro-quinolin-3-yl](pyridine-2-sulfonyl)-amino]-methyl\}-piperidine-1-carboxylic Acid Methyl Ester (162)

${ }^{1} \mathrm{H}$ NMR $\left(300 \mathrm{MHz}\right.$, methanol- $\left.d_{4}\right) \delta 8.94(\mathrm{~d}, J=0.9 \mathrm{~Hz}, 1 \mathrm{H}), 8.70(\mathrm{~d}, J=7.5 \mathrm{~Hz}, 1 \mathrm{H}), 8.08$ $(\mathrm{td}, J=1.8,7.8 \mathrm{~Hz}, 1 \mathrm{H}), 8.0(\mathrm{dt}, J=1.2,7.8 \mathrm{~Hz}, 1 \mathrm{H}), 7.66(\mathrm{ddd}, J=1.5,4.8,7.5 \mathrm{~Hz}, 1 \mathrm{H}), 7.43$ $(\mathrm{d}, J=1.2 \mathrm{~Hz}, 1 \mathrm{H}), 7.38(\mathrm{dd}, J=2.1,8.7 \mathrm{~Hz}, 1 \mathrm{H}), 7.33(\mathrm{~s}, 1 \mathrm{H}), 6.81(\mathrm{~d}, J=8.7 \mathrm{~Hz}, 1 \mathrm{H}), 4.84$ $(\mathrm{d}, J=17.7 \mathrm{~Hz}, 1 \mathrm{H}), 4.68(\mathrm{~d}, J=17.7 \mathrm{~Hz}, 1 \mathrm{H}), 4.38-4.51(\mathrm{~m}, 1 \mathrm{H}), 4.08-4.16(\mathrm{~m}, 2 \mathrm{H}), 3.92$ (s, 3H), 3.65 (s, 3H), 3.51-3.62 (m, 2H), 3.21-3.26 (m, 2H), 3.11-3.18 (m, 1H), 2.79-2.87 (m, $3 \mathrm{H}), 1.68-1.88$ (m, 3H), 1.05-1.17 (m, 2H). MS m/z $564.4\left(\mathrm{M}+\mathrm{H}^{+}\right)$.

4-\{[[6-Cyano-1-(3-methyl-3H-imidazol-4-ylmethyl)-1,2,3,4-tetrahydro-quinolin-3-yl](pyridine-2-sulfonyl)-amino]-methyl\}-piperidine-1-carboxylic Acid Isobutyl Ester (167)

${ }^{1} \mathrm{H}$ NMR $\left(300 \mathrm{MHz}\right.$, methanol- $\left.d_{4}\right) \delta 8.94(\mathrm{~d}, J=0.9 \mathrm{~Hz}, 1 \mathrm{H}), 8.70(\mathrm{~d}, J=7.5 \mathrm{~Hz}, 1 \mathrm{H}), 8.08$ $(\mathrm{td}, J=1.8,7.8 \mathrm{~Hz}, 1 \mathrm{H}), 8.0(\mathrm{dt}, J=1.2,7.8 \mathrm{~Hz}, 1 \mathrm{H}), 7.66(\mathrm{ddd}, J=1.5,4.8,7.5 \mathrm{~Hz}, 1 \mathrm{H}), 7.43$ $(\mathrm{d}, J=1.2 \mathrm{~Hz}, 1 \mathrm{H}), 7.38(\mathrm{dd}, J=2.1,8.7 \mathrm{~Hz}, 1 \mathrm{H}), 7.33(\mathrm{~s}, 1 \mathrm{H}), 6.81(\mathrm{~d}, J=8.7 \mathrm{~Hz}, 1 \mathrm{H}), 4.84$ $(\mathrm{d}, J=17.7 \mathrm{~Hz}, 1 \mathrm{H}), 4.68(\mathrm{~d}, J=17.7 \mathrm{~Hz}, 1 \mathrm{H}), 4.38-4.51(\mathrm{~m}, 1 \mathrm{H}), 4.08-4.16(\mathrm{~m}, 2 \mathrm{H}), 3.92$ (s, 3H), $3.85(\mathrm{~d}, J=6.6 \mathrm{~Hz}, 2 \mathrm{H}), 3.51-3.62(\mathrm{~m}, 2 \mathrm{H}), 3.21-3.26(\mathrm{~m}, 2 \mathrm{H}), 3.11-3.18(\mathrm{~m}, 1 \mathrm{H})$, 2.79-2.87 (m, 3H), 1.91-1.99 (m, 1H), 1.68-1.88, (m, 3H), 1.05-1.17 (m, 2H), $0.95(\mathrm{~d}, J=$ $6.9 \mathrm{~Hz}, 6 \mathrm{H}) . \mathrm{MS} m / z 606.5\left(\mathrm{M}+\mathrm{H}^{+}\right)$.

Pyridine-2-sulfonic Acid [6-Cyano-1-(3-methyl-3H-imidazol-4-ylmethyl)-1,2,3,4-tetrahydroquinolin-3-yl]-[1-(2,2-dimethyl-propionyl)-piperidin-4-ylmethyl]-amide (150)

${ }^{1} \mathrm{H} \mathrm{NMR}\left(500 \mathrm{MHz}\right.$, methanol- $\left.d_{4}\right) \delta 8.94(\mathrm{~s}, 1 \mathrm{H}), 8.71(\mathrm{~d}, J=6 \mathrm{~Hz}, 1 \mathrm{H}), 8.09(\mathrm{td}, J=1,8 \mathrm{~Hz}$, $1 \mathrm{H}), 8.01(\mathrm{dt}, J=1,8 \mathrm{~Hz}, 1 \mathrm{H}), 7.66(\mathrm{ddd}, J=1.5,5,7.5 \mathrm{~Hz}, 1 \mathrm{H}), 7.43(\mathrm{~s}, 1 \mathrm{H}), 7.38$ (dd, $J=$ $2,8 \mathrm{~Hz}, 1 \mathrm{H}), 7.33(\mathrm{~s}, 1 \mathrm{H}), 6.82(\mathrm{~d}, J=9 \mathrm{~Hz}, 1 \mathrm{H}), 4.82(\mathrm{~d}, J=18.5 \mathrm{~Hz}, 1 \mathrm{H}), 4.69(\mathrm{~d}, J=18.5$ $\mathrm{Hz}, 1 \mathrm{H}), 4.38-4.45(\mathrm{~m}, 3 \mathrm{H}), 3.92(\mathrm{~s}, 3 \mathrm{H}), 3.61-3.66(\mathrm{~m}, 1 \mathrm{H}), 3.52-3.55(\mathrm{~m}, 1 \mathrm{H}), 3.15-3.30$ (m, 3H), 2.76-2.88 (m, 3H), 1.93-1.97 (m, 1H), 1.77-1.85 (m, 2H), 1.27 (s, 9H), 1.07-1.15 $(\mathrm{m}, 2 \mathrm{H}) . \mathrm{MS} m / z 590.6\left(\mathrm{M}+\mathrm{H}^{+}\right)$.

Pyridine-2-sulfonic Acid [6-Cyano-1-(3-methyl-3H-imidazol-4-ylmethyl)-1,2,3,4-tetrahydroquinolin-3-yl]-[1-(3,3,3-trifluoro-propionyl)-piperidin-4-ylmethyl]-amide (155)

${ }^{1} \mathrm{H}$ NMR $\left(300 \mathrm{MHz}\right.$, methanol- $\left.d_{4}\right) \delta 8.85(\mathrm{~s}, 1 \mathrm{H}), 8.67(\mathrm{~d}, J=6 \mathrm{~Hz}, 1 \mathrm{H}), 8.09(\mathrm{td}, J=1,8 \mathrm{~Hz}$, $1 \mathrm{H}), 8.01(\mathrm{dt}, J=1,8 \mathrm{~Hz}, 1 \mathrm{H}), 7.60(\mathrm{~m}, 1 \mathrm{H}), 7.43(\mathrm{~s}, 1 \mathrm{H}), 7.38(\mathrm{~d}, J=8 \mathrm{~Hz}, 1 \mathrm{H}), 7.23(\mathrm{~s}, 1 \mathrm{H})$, $6.82(\mathrm{~d}, J=9 \mathrm{~Hz}, 1 \mathrm{H}), 4.82(\mathrm{~d}, J=18.5 \mathrm{~Hz}, 1 \mathrm{H}), 4.69(\mathrm{~d}, J=18.5 \mathrm{~Hz}, 1 \mathrm{H}), 4.38-4.45(\mathrm{~m}, 3$ $\mathrm{H}), 3.92(\mathrm{~s}, 3 \mathrm{H}), 3.61-3.66(\mathrm{~m}, 1 \mathrm{H}), 3.50-3.52(\mathrm{~m}, 1 \mathrm{H}), 3.12-3.28(\mathrm{~m}, 5 \mathrm{H}), 2.71-2.81(\mathrm{~m}$, $3 \mathrm{H}), 1.93-1.97(\mathrm{~m}, 1 \mathrm{H}), 1.72-1.80(\mathrm{~m}, 2 \mathrm{H}), 1.03-1.12(\mathrm{~m}, 2 \mathrm{H})$. MS m/z $616.4\left(\mathrm{M}+\mathrm{H}^{+}\right)$. 


\section{Pyridine-2-sulfonic Acid [6-Cyano-1-(3-methyl-3H-imidazol-4-ylmethyl)-1,2,3,4-tetrahydro- quinolin-3-yl]-(1-methanesulfonyl-piperidin-4-ylmethyl)-amide (177)}

${ }^{1} \mathrm{H}$ NMR $\left(300 \mathrm{MHz}\right.$, methanol- $\left.d_{4}\right) \delta 8.93(\mathrm{~d}, J=1.2 \mathrm{~Hz}, 1 \mathrm{H}), 8.71(\mathrm{~d}, J=7.5 \mathrm{~Hz}, 1 \mathrm{H}), 8.08$ $(\mathrm{dt}, J=1.8,7.8 \mathrm{~Hz}, 1 \mathrm{H}), 8.02(\mathrm{td}, J=1.2,8.1 \mathrm{~Hz}, 1 \mathrm{H}), 7.66(\mathrm{ddd}, J=1.5,4.8,7.5 \mathrm{~Hz}, 1 \mathrm{H})$, $7.42(\mathrm{~d}, J=1.5 \mathrm{~Hz}, 1 \mathrm{H}), 7.38(\mathrm{dd}, J=2.1,8.7 \mathrm{~Hz}, 1 \mathrm{H}), 7.33(\mathrm{~s}, 1 \mathrm{H}), 6.80(\mathrm{~d}, J=8.7 \mathrm{~Hz}, 1 \mathrm{H})$, $4.8(\mathrm{~d}, J=17.7 \mathrm{~Hz}, 1 \mathrm{H}), 4.68(\mathrm{~d}, J=17.7 \mathrm{~Hz}, 1 \mathrm{H}), 4.37-4.48(\mathrm{~m}, 1 \mathrm{H}), 3.91(\mathrm{~s}, 3 \mathrm{H}), 3.69-3.72$ $(\mathrm{m}, 2 \mathrm{H}), 3.58-3.65(\mathrm{~m}, 1 \mathrm{H}), 3.49-3.55(\mathrm{~m}, 1 \mathrm{H}), 3.22-3.28(\mathrm{~m}, 2 \mathrm{H}), 3.13-3.20(\mathrm{~m}, 1 \mathrm{H}), 2.85-$ $2.89(\mathrm{~m}, 1 \mathrm{H}), 2.82(\mathrm{~s}, 3 \mathrm{H}), 2.64-2.74(\mathrm{~m}, 2 \mathrm{H}), 1.75-1.93(\mathrm{~m}, 3 \mathrm{H}), 1.17-1.35(\mathrm{~m}, 2 \mathrm{H}) . \mathrm{MS} \mathrm{m} /$ $z 584.4\left(\mathrm{M}+\mathrm{H}^{+}\right)$.

\section{Pyridine-2-sulfonic Acid (1-Benzenesulfonyl-piperidin-4-yl-methyl)-[6-cyano-1-(3- methyl-3H-imidazol-4-ylmethyl)-1,2,3,4-tetrahydro-quinolin-3-yl]-amide (180)}

${ }^{1} \mathrm{H}$ NMR $\left(300 \mathrm{MHz}\right.$, methanol- $\left.d_{4}\right) \delta 8.93(\mathrm{~d}, J=1.2 \mathrm{~Hz}, 1 \mathrm{H}), 8.71(\mathrm{~d}, J=7.5 \mathrm{~Hz}, 1 \mathrm{H}), 8.08$ (dt, $J=1.8,7.8 \mathrm{~Hz}, 1 \mathrm{H}), 8.02(\mathrm{td}, J=1.2,8.1 \mathrm{~Hz}, 1 \mathrm{H}), 7.56-7.78(\mathrm{~m}, 6 \mathrm{H}), 7.32(\mathrm{~s}, 1 \mathrm{H}), 7.29$ $(\mathrm{d}, J=8.7 \mathrm{~Hz}, 1 \mathrm{H}), 7.21(\mathrm{~s}, 1 \mathrm{H}), 6.80(\mathrm{~d}, J=8.7 \mathrm{~Hz}, 1 \mathrm{H}), 4.75(\mathrm{~d}, J=17.7 \mathrm{~Hz}, 1 \mathrm{H}), 4.60(\mathrm{~d}$, $J=17.7 \mathrm{~Hz}, 1 \mathrm{H}), 4.37-4.48(\mathrm{~m}, 1 \mathrm{H}), 3.91(\mathrm{~s}, 3 \mathrm{H}), 3.69-3.72(\mathrm{~m}, 2 \mathrm{H}), 3.58-3.65(\mathrm{~m}, 1 \mathrm{H})$, 3.49-3.55 (m, 1H), 3.22-3.28 (m, 2H), 3.13-3.20 (m, 1H), 2.85-2.89 (m, 1H), 2.64-2.74 (m, $2 \mathrm{H}), 1.75-1.93(\mathrm{~m}, 3 \mathrm{H}), 1.17-1.35(\mathrm{~m}, 2 \mathrm{H})$. MS $m / z 646.5\left(\mathrm{M}+\mathrm{H}^{+}\right)$.

\section{4-\{[[6-Cyano-1-(3-methyl-3H-imidazol-4-ylmethyl)-1,2,3,4-tetrahydro-quinolin-3-yl]- (pyridine-2-sulfonyl)-amino]-methyl\}-piperidine-1-carboxylic Acid Ethylamide (170)}

${ }^{1} \mathrm{H}$ NMR $\left(300 \mathrm{MHz}\right.$, methanol- $\left.d_{4}\right) \delta 8.93(\mathrm{~s}, 1 \mathrm{H}), 8.70(\mathrm{~d}, J=7.2 \mathrm{~Hz}, 1 \mathrm{H}), 8.04-8.10(\mathrm{~m}, 1 \mathrm{H})$, 8.99-8.02 (m, 1H), $7.66(\mathrm{ddd}, J=1.5,4.8,7.5 \mathrm{~Hz}, 1 \mathrm{H}), 7.42(\mathrm{~s}, 1 \mathrm{H}), 7.39(\mathrm{dd}, J=2.1,8.4 \mathrm{~Hz}$, $1 \mathrm{H}), 7.33(\mathrm{~s}, 1 \mathrm{H}), 6.82(\mathrm{~d}, J) 8.7 \mathrm{~Hz}, 1 \mathrm{H}), 4.8(\mathrm{~d}, J=17.7 \mathrm{~Hz}, 1 \mathrm{H}), 4.69(\mathrm{~d}, J=17.7 \mathrm{~Hz}, 1 \mathrm{H})$, 4.38-4.47 (m, 1H), 3.98-4.02 (m, 2H), 3.91 (s, 3H), $3.62(\mathrm{t}, J=10.8 \mathrm{~Hz}, 1 \mathrm{H}), 3.54-3.56(\mathrm{~m}$, $1 \mathrm{H}), 3.12-3.29(\mathrm{~m}, 5 \mathrm{H}), 2.81-2.90(\mathrm{~m}, 1 \mathrm{H}), 2.66-2.74(\mathrm{~m}, 2 \mathrm{H}), 1.65-1.87$ (m, 3H), 1.17-1.18 $(\mathrm{m}, 2 \mathrm{H}), 1.10(\mathrm{t}, J=7.2 \mathrm{~Hz}, 3 \mathrm{H})$. MS $m / z 577.5\left(\mathrm{M}+\mathrm{H}^{+}\right)$.

[2-(4-\{[[6-Cyano-1-(3-methyl-3H-imidazol-4-ylmethyl)-1,2,3,4-tetrahydro-quinolin-3-yl](pyridine-2-sulfonyl)-amino]-methyl\}-piperidin-1-yl)-2-oxo-ethyl]-carbamic Acid tert-Butyl Ester (157)

A mixture of 54 (22 mg, $0.044 \mathrm{mmol})$, Boc-Gly-OH (9.2 mg, $0.051 \mathrm{mmol})$, dicyclohexylcarbodiimide (10 $\mathrm{mg}, 0.051 \mathrm{mmol})$, 4-dimethylamino-pyridine $(2 \mathrm{mg})$, and dichloromethane $(5 \mathrm{~mL})$ was stirred at room temperature for $18 \mathrm{~h}$. Upon completion of reaction, the mixture was filtered to remove dicyclohexylurea, and the residue was washed with dichloromethane $(5 \mathrm{~mL})$. The filtrate and washings were combined and evaporated to dryness in Vacuo. The residue was purified by HPLC to afford $15 \mathrm{mg}$ (54\%) of $\mathbf{1 5 7}$ as the trifluoroacetate salt. ${ }^{1} \mathrm{H}$ NMR $\left(500 \mathrm{MHz}\right.$, methanol- $\left.d_{4}\right) \delta 8.94(\mathrm{~s}, 1 \mathrm{H}), 8.71(\mathrm{~d}, J=7.5 \mathrm{~Hz}, 1 \mathrm{H})$, 8.06-8.12 (m, 1H), 7.88-8.01 (m, 1H), 7.65-7.68 (m, 1H), $7.42(\mathrm{~s}, 1 \mathrm{H}), 7.38(\mathrm{dd}, J=1.5,8.5$ $\mathrm{Hz}, 1 \mathrm{H}), 7.33(\mathrm{~s}, 1 \mathrm{H}), 6.81(\mathrm{~d}, J=8 \mathrm{~Hz}, 1 \mathrm{H}), 4.81(\mathrm{~d}, J=18 \mathrm{~Hz}, 1 \mathrm{H}), 4.69(\mathrm{~d}, J=18 \mathrm{~Hz}, 1 \mathrm{H})$, 4.35-4.50 (m, 3H), $3.92(\mathrm{~s}, 3 \mathrm{H}), 3.80-3.92(\mathrm{~m}, 2 \mathrm{H}), 3.59-3.63(\mathrm{~m}, 1 \mathrm{H}), 3.52-3.54(\mathrm{~m}, 1 \mathrm{H})$, $3.13-3.33(\mathrm{~m}, 3 \mathrm{H}), 3.00-3.12(\mathrm{~m}, 1 \mathrm{H}), 2.82-2.88(\mathrm{~m}, 1 \mathrm{H}), 2.57-2.65(\mathrm{~m}, 1 \mathrm{H}), 1.72-1.95(\mathrm{~m}$, $3 \mathrm{H}), 1.46(\mathrm{~s}, 9 \mathrm{H}), 1.06-1.25(\mathrm{~m}, 2 \mathrm{H})$. MS m/z $663.6\left(\mathrm{M}+\mathrm{H}^{+}\right)$.

\section{General Procedure for the Synthesis of Compounds According to Scheme 3}

To a solution of 6-cyano-1,2,3,4-tetrahydro-quinolin-3-ylamine hydrochloride $\mathbf{3}$ (4.18 g, 20 $\mathrm{mmol})$ and $N, N$-diisopropylethyl amine $(5.1 \mathrm{~g}, 40 \mathrm{mmol})$ in $25 \mathrm{~mL}$ of anhydrous dichloromethane was added benzyl chloroformate $(5.1 \mathrm{~g}, 30 \mathrm{mmol})$ at $0{ }^{\circ} \mathrm{C}$. After stirring at ambient temperature for $5 \mathrm{~h}$, the reaction mixture was quenched with water. The mixture was partitioned between water and ethyl acetate. The organic layer was washed with brine, dried 
over sodium sulfate, and evaporated under reduce pressure. Purification by flash chromatography gave $\mathbf{9}(5.49 \mathrm{~g} 89 \%)$ as a white foam.

To a solution of $9(5 \mathrm{~g}, 16.2 \mathrm{mmol})$ and trifluoroacetic acid $(14 \mathrm{~mL})$ in $20 \mathrm{~mL}$ of dichloroethane $(14 \mathrm{~mL})$ at room temperature under nitrogen was added 3-methyl-3H-imidazole-4carboxaldehyde $(5.37 \mathrm{~g}, 48.6 \mathrm{mmol})$. The mixture was stirred for $1 \mathrm{~h}$ at room temperature, and then triethylsilane $(7.75 \mathrm{~mL}, 48.6 \mathrm{mmol})$ was added dropwise. The mixture was heated in an oil bath at $45{ }^{\circ} \mathrm{C}$ for $15 \mathrm{~h}$. The volatile materials were removed under vacuum. The reaction mixture was diluted with ethyl acetate and washed with aqueous $\mathrm{NaHCO}_{3}$, water, and brine solution. The organic layer was dried over $\mathrm{MgSO}_{4}$, filtered, and concentrated. The crude residue was purified on a flash silica column to afford $\mathbf{1 0}(3.1 \mathrm{~g}, 47 \%)$.

A solution of [6-cyano-1-(3-methyl-3H-imidazol-4-ylmethyl)-1,2,3,4-tetrahydro-quinolin-3yl]-carbamic acid benzyl ester 10 (1.3 g, $3 \mathrm{mmol}), 15 \mathrm{~mL}$ methanol, and $10 \% \mathrm{Pd} / \mathrm{C}$ catalyst $(0.5 \mathrm{~g})$ was stirred under atmospheric pressure of hydrogen for $4 \mathrm{~h}$. The catalyst was filtered off, and the filtrate was concentrated to give $\mathbf{1 1}$ as an off-white foam (790 mg, 92\%).

To a suspension of $11(0.55 \mathrm{~g}, 0.2 \mathrm{mmol})$ and $\mathrm{Cs}_{2} \mathrm{CO}_{3}(1.34 \mathrm{~g}, 0.4 \mathrm{mmol})$ in dry DMF ( $\left.3 \mathrm{~mL}\right)$ was added the appropriate alkyl halide $(0.2 \mathrm{mmol})$, and the mixture was stirred at room temperature overnight under argon. After addition of water $(10 \mathrm{~mL})$, the solution was extracted with ethyl acetate $(3 \times 10 \mathrm{~mL})$. The organic layer was extracted with brine $(3 \times 10 \mathrm{~mL})$. The combined organic layers were dried over $\mathrm{MgSO}_{4}$ and evaporated under reduce pressure. The residue was purified by HPLC. Appropriate fractions were collected, and the pure product 12 was obtained as the TFA salt.

Compound $12(0.1 \mathrm{mmol})$ dissolved in dichloromethane $(1.5 \mathrm{~mL})$ together with $\mathrm{N}, \mathrm{N}$ diisopropylethyl amine $(0.2 \mathrm{mmol})$ was cooled to $0{ }^{\circ} \mathrm{C}$. Acetyl chloride or sulfonyl chloride $(0.1 .2 \mathrm{mmol})$ dissolved in dichloromethane $(0.5 \mathrm{~mL})$ was added dropwise, and the mixture was stirred for $1 \mathrm{~h}$. Aqueous $\mathrm{NH}_{4} \mathrm{OH}(1 \mathrm{~mL})$ was added to the reaction mixture. After being stirred for an additional $15 \mathrm{~min}$, the reaction mixture was diluted with ethyl acetate $(50 \mathrm{~mL})$ and washed with brine solution. The organic layer was dried over $\mathrm{MgSO}_{4}$ and evaporated under reduced pressure, yielding the crude amide 13, which was purified by HPLC. The appropriate fractions were combined, concentrated in vacuo, and lyophilized to give the corresponding compounds as the trifluoroacetate salt.

\section{(6-Cyano-1,2,3,4-tetrahydro-quinolin-3-yl)-carbamic Acid Benzyl Ester (9)}

${ }^{1} \mathrm{H}$ NMR $\left(300 \mathrm{MHz}\right.$, methanol- $\left.d_{4}\right) \delta 7.02-7.15(\mathrm{~m}, 7 \mathrm{H}), 6.58(\mathrm{~d}, J=8 \mathrm{~Hz}, 1 \mathrm{H}), 5.09$ (s, 2H), 3.94-3.98 (m, 1H), $3.45(\mathrm{~m}, 1 \mathrm{H}), 3.20(\mathrm{~m}, 1 \mathrm{H}), 2.99(\mathrm{dd} J=4.2,16.5 \mathrm{~Hz}, 1 \mathrm{H}), 2.74$ (ddd, $J=$ $2.1,4.8,16.5 \mathrm{~Hz}, 1 \mathrm{H}) . \mathrm{MS} m / z 308.2\left(\mathrm{M}+\mathrm{H}^{+}\right)$.

\section{[6-Cyano-1-(3-methyl-3H-imidazol-4-ylmethyl)-1,2,3,4-tetrahydro-quinolin-3-yl]-carbamic Acid Benzyl Ester (10)}

${ }^{1} \mathrm{H}$ NMR $\left(300 \mathrm{MHz}, \mathrm{CD}_{3} \mathrm{OD}\right) \delta 7.61(\mathrm{~s}, 1 \mathrm{H}), 7.31-7.38(\mathrm{~m}, 7 \mathrm{H}) 6.87(\mathrm{~d}, J=8.7 \mathrm{~Hz}, 1 \mathrm{H}), 6.83$ (s, 1H), $5.07(\mathrm{~s}, 2 \mathrm{H}), 4.59(\mathrm{~s}, 2 \mathrm{H}), 4.00-4.02(\mathrm{~m}, 1 \mathrm{H}), 3.64(\mathrm{~s}, 3 \mathrm{H}), 3.36(\mathrm{dd}, J=2.7,12 \mathrm{~Hz}$, $1 \mathrm{H}), 3.23(\mathrm{dd}, J=4.5,12.1 \mathrm{~Hz}, 1 \mathrm{H}), 2.03(\mathrm{dd} J=5.1,15.9 \mathrm{~Hz}, 1 \mathrm{H}), 2.71(\mathrm{dd}, J=4.8,16.2 \mathrm{~Hz}$, 1H). MS m/z $402.2\left(\mathrm{M}+\mathrm{H}^{+}\right)$.

\section{3-Amino-1-(3-methyl-3H-imidazol-4-ylmethyl)-1,2,3,4-tetrahydro-quinoline-6-carbonitrile} (11)

${ }^{1} \mathrm{H}$ NMR $\left(300 \mathrm{MHz}\right.$, methanol- $\left.d_{4}\right) \delta 7.61(\mathrm{~s}, 1 \mathrm{H}), 7.32(\mathrm{dd}, J=2.1,8.4 \mathrm{~Hz}, 1 \mathrm{H}), 7.29(\mathrm{~s}, 1 \mathrm{H})$, $6.83(\mathrm{~d}, J=8.4 \mathrm{~Hz}, 1 \mathrm{H}), 6.80(\mathrm{~s}, 1 \mathrm{H}), 4.64(\mathrm{~d}, J=16.5 \mathrm{~Hz}, 1 \mathrm{H}), 4.58(\mathrm{~d}, J=16.5 \mathrm{~Hz}, 1 \mathrm{H})$, 
$3.57(\mathrm{~s}, 3 \mathrm{H}), 3.42(\mathrm{dd}, J=2.4,11.7 \mathrm{~Hz}, 1 \mathrm{H}), 3.35(\mathrm{~m}, 1 \mathrm{H}), 3.21(\mathrm{~m}, 1 \mathrm{H}), 2.98(\mathrm{dd}, J=4.1$, $16.5 \mathrm{~Hz}, 1 \mathrm{H}), 2.67(\mathrm{dd}, J=4.8,16.4 \mathrm{~Hz}, 1 \mathrm{H})$. MS $m / z 268.3\left(\mathrm{M}+\mathrm{H}^{+}\right)$.

3-(2-Fluoro-benzylamino)-1-(3-methyl-3H-imidazol-4-ylmethyl)-1,2,3,4-tetrahydroquinoline-6-carbonitrile Trifluoroacetate Salt (12)

${ }^{1} \mathrm{H}$ NMR $\left(300 \mathrm{MHz}\right.$, methanol- $\left.d_{4}\right) \delta 8.83(\mathrm{~s}, 1 \mathrm{H}), 7.20-7.48(\mathrm{~m}, 5 \mathrm{H}), 7.12-7.17(\mathrm{~m}, 2 \mathrm{H}), 6.82$ $(\mathrm{d}, J=8.5 \mathrm{~Hz}, 1 \mathrm{H}), 4.74(\mathrm{~d}, J=17.4 \mathrm{~Hz}, 1 \mathrm{H}), 4.60(\mathrm{~d}, J=17.4 \mathrm{~Hz}, 1 \mathrm{H}), 4.35(\mathrm{~s}, 2 \mathrm{H}), 3.88(\mathrm{~m}$, $1 \mathrm{H}), 3.75(\mathrm{~s}, 3 \mathrm{H}), 3.74(\mathrm{dd}, J=2.1,14.1 \mathrm{~Hz}, 1 \mathrm{H}), 3.49(\mathrm{dd}, J=4.8,14.5 \mathrm{~Hz}, 1 \mathrm{H}), 3.31(\mathrm{dd}$, $J=4.5,16.5 \mathrm{~Hz}, 1 \mathrm{H}), 2.67(\mathrm{dd}, J=6.6,16.5 \mathrm{~Hz}, 1 \mathrm{H}) . \mathrm{MS} m / z 268.3\left(\mathrm{M}+\mathrm{H}^{+}\right)$.

4-\{[6-Cyano-1-(3-methyl-3H-imidazol-4-ylmethyl)-1,2,3,4-tetrahydro-quinolin-3-ylamino]methyl\}-piperidine-1-carboxylic Acid Methyl Ester (12)

${ }^{1} \mathrm{H}$ NMR $\left(300 \mathrm{MHz}\right.$, methanol- $\left.d_{4}\right) \delta 8.87(\mathrm{~s}, 1 \mathrm{H}), 7.43(\mathrm{~s}, 1 \mathrm{H}), 7.38(\mathrm{dd}, J=2.1,8.7 \mathrm{~Hz}, 1 \mathrm{H})$, $7.33(\mathrm{~s}, 1 \mathrm{H}), 6.81(\mathrm{~d}, J=8.7 \mathrm{~Hz}, 1 \mathrm{H}), 4.84(\mathrm{~d}, J=17.7 \mathrm{~Hz}, 1 \mathrm{H}), 4.68(\mathrm{~d}, J=17.7 \mathrm{~Hz}, 1 \mathrm{H})$, $3.89-4.08(\mathrm{~m}, 3 \mathrm{H}), 3.82(\mathrm{~s}, 3 \mathrm{H}), 3.65(\mathrm{~s}, 3 \mathrm{H}), 3.51-3.62(\mathrm{~m}, 2 \mathrm{H}), 3.21-3.26(\mathrm{~m}, 2 \mathrm{H}), 3.11-$ $3.18(\mathrm{~m}, 1 \mathrm{H}), 2.79-2.87(\mathrm{~m}, 3 \mathrm{H}), 1.68-1.88,(\mathrm{~m}, 3 \mathrm{H}), 1.05-1.17(\mathrm{~m}, 2 \mathrm{H}) . \mathrm{MS} m / z 423.4(\mathrm{M}+$ $\left.\mathrm{H}^{+}\right)$.

$\mathrm{N}$-[6-Cyano-1-(3-methyl-3H-imidazol-4-ylmethyl)-1,2,3,4-tetrahydro-quinolin-3-yl]- $\mathrm{N}$-(2fluoro-benzyl)-4-methoxy-benzene-sulfonamide (215)

${ }^{1} \mathrm{H}$ NMR $\left(300 \mathrm{MHz}\right.$, methanol- $\left.d_{4}\right) \delta 8.91(\mathrm{~s}, 1 \mathrm{H}), 7.83(\mathrm{~d}, J=9.1 \mathrm{~Hz}, 2 \mathrm{H}), 7.49-7.52(\mathrm{~m}, 1 \mathrm{H})$, 7.28-7.33 (m, 3H), 7.16-7.19 (m, 2H), 7.1 (d, $J=9.0 \mathrm{~Hz}, 2 \mathrm{H}), 6.96-7.03(\mathrm{~m}, 1 \mathrm{H}), 6.71(\mathrm{~d}, J$ $=9.0 \mathrm{~Hz}, 1 \mathrm{H}), 4.30-4.61(\mathrm{~m}, 5 \mathrm{H}), 3.91(\mathrm{~s}, 3 \mathrm{H}), 3.81(\mathrm{~s}, 3 \mathrm{H}), 3.30-3.39(\mathrm{~m}, 2 \mathrm{H}), 2.94-3.04$ (m, 1H), $2.75(\mathrm{dd}, J=4.8,15.6 \mathrm{~Hz}, 1 \mathrm{H}) . \mathrm{MS} m / z 546.5\left(\mathrm{M}+\mathrm{H}^{+}\right)$.

1-Methyl-1 H-pyrazole-4-sulfonic Acid [6-Cyano-1-(3-methyl-3H-imidazol-4-ylmethyl)-1,2,3,4tetrahydro-quinolin-3-yl]-(2-fluoro-benzyl)-amide (213)

${ }^{1} \mathrm{H}$ NMR $\left(300 \mathrm{MHz}\right.$, methanol- $\left.d_{4}\right) \delta 8.91(\mathrm{~s}, 1 \mathrm{H}), 8.19(\mathrm{~s}, 1 \mathrm{H}), 7.82(\mathrm{~s}, 1 \mathrm{H}), 7.56-7.61(\mathrm{~m}$, $1 \mathrm{H}), 7.27-7.36(\mathrm{~m}, 4 \mathrm{H}), 7.14-7.22(\mathrm{~m}, 1 \mathrm{H}), 7.01-7.09(\mathrm{~m}, 1 \mathrm{H}), 6.72(\mathrm{~d}, J=8.4 \mathrm{~Hz}, 1 \mathrm{H}), 4.32-$ $4.59(\mathrm{~m}, 5 \mathrm{H}), 3.96(\mathrm{~s}, 3 \mathrm{H}), 3.82(\mathrm{~s}, 3 \mathrm{H}), 3.30-3.39(\mathrm{~m}, 2 \mathrm{H}), 3.03-3.12(\mathrm{~m}, 1 \mathrm{H}), 2.86(\mathrm{dd}, J=$ 4.8, $15.6 \mathrm{~Hz}, 1 \mathrm{H}) . \mathrm{MS} \mathrm{m} / \mathrm{z} 520.4\left(\mathrm{M}+\mathrm{H}^{+}\right)$.

3-Methyl-3H-imidazole-4-sulfonic Acid [6-Cyano-1-(3-methyl-3H-imidazol-4ylmethyl)-1,2,3,4-tetrahydro-quinolin-3-yl]-(4-methanesulfonyl-benzyl)-amide (221)

${ }^{1} \mathrm{H}$ NMR $\left(300 \mathrm{MHz}\right.$, methanol- $\left.d_{4}\right) \delta 8.89(\mathrm{~s}, 1 \mathrm{H}), 7.93(\mathrm{~d}, J=8.2 \mathrm{~Hz}, 2 \mathrm{H}), 7.83(\mathrm{~s}, 1 \mathrm{H}), 7.76$ $(\mathrm{s}, 1 \mathrm{H}), 7.62(\mathrm{~d}, J=8.4 \mathrm{~Hz}, 2 \mathrm{H}), 7.31-7.35(\mathrm{~m}, 2 \mathrm{H}), 7.26(\mathrm{~s}, 1 \mathrm{H}), 6.68(\mathrm{~d}, J=8.7 \mathrm{~Hz}, 1 \mathrm{H})$, 4.39-4.68 (m, 5H), 3.81 (s, 6H), 3.40-3.46 (m, 2H), $3.15(\mathrm{~s}, 3 \mathrm{H}), 3.03-3.11(\mathrm{~m}, 1 \mathrm{H}), 2.86(\mathrm{~m}$, $1 \mathrm{H})$. MS $m / z 580.5\left(\mathrm{M}+\mathrm{H}^{+}\right)$.

$\mathrm{N}$-[6-Cyano-1-(3-methyl-3H-imidazol-4-ylmethyl)-1,2,3,4-tetrahydro-quinolin-3-yl]- $\mathrm{N}$-(4methanesulfonyl-benzyl)-benzene-sulfonamide (218)

${ }^{1} \mathrm{H}$ NMR $\left(300 \mathrm{MHz}\right.$, methanol- $\left.d_{4}\right) \delta 8.91(\mathrm{~s} 1 \mathrm{H}), 7.92-7.96(\mathrm{~m}, 4 \mathrm{H}), 7.67-7.72(\mathrm{~m}, 5 \mathrm{H}), 7.31$ $(\mathrm{dd}, J=2.1,8.4 \mathrm{~Hz}, 1 \mathrm{H}), 7.27(\mathrm{~s}, 1 \mathrm{H}), 7.16(\mathrm{~s}, 1 \mathrm{H}), 6.63(\mathrm{~d}, J=8.4 \mathrm{~Hz}, 1 \mathrm{H}), 4.35-4.70(\mathrm{~m}$, $5 \mathrm{H}), 3.82(\mathrm{~s}, 3 \mathrm{H}), 3.41-3.46(\mathrm{~m}, 2 \mathrm{H}), 3.17(\mathrm{~s}, 3 \mathrm{H}), 2.95-3.05(\mathrm{~m}, 1 \mathrm{H}), 2.79-2.82(\mathrm{~m}, 1 \mathrm{H}) . \mathrm{MS}$ $\mathrm{m} / \mathrm{z} 576.6\left(\mathrm{M}+\mathrm{H}^{+}\right)$.

4-(\{[6-Cyano-1-(3-methyl-3H-imidazol-4-ylmethyl)-1,2,3,4-tetrahydro-quinolin-3-yl]ethanesulfonyl-amino\}-methyl)-piperidine-1-carboxylic Acid Methyl Ester (200)

${ }^{1} \mathrm{H}$ NMR $\left(300 \mathrm{MHz}\right.$, methanol- $\left.d_{4}\right) \delta 8.93(\mathrm{~s}, 1 \mathrm{H}), 7.42(\mathrm{~s}, 1 \mathrm{H}), 7.42(\mathrm{~s}, 1 \mathrm{H}), 7.33(\mathrm{dd}, J=1.8$, $8.4 \mathrm{~Hz}, 1 \mathrm{H}), 6.82(\mathrm{~d}, J=8.7 \mathrm{~Hz}, 1 \mathrm{H}), 4.79(\mathrm{~d}, J=18 \mathrm{~Hz}, 1 \mathrm{H}), 4.70(\mathrm{~d}, J=18 \mathrm{~Hz}, 1 \mathrm{H}), 4.05-$ 
$4.18(\mathrm{~m}, 3 \mathrm{H}), 3.92(\mathrm{~s}, 3 \mathrm{H}), 3.65(\mathrm{~s}, 3 \mathrm{H}), 3.51-3.56(\mathrm{~m}, 2 \mathrm{H}), 3.12-3.24(\mathrm{~m}, 6 \mathrm{H}), 2.71-2.78(\mathrm{~m}$, $2 \mathrm{H}), 1.72-1.84(\mathrm{~m}, 3 \mathrm{H}), 1.34$ (t, $J=7.5 \mathrm{~Hz}, 3 \mathrm{H}), 1.05-1.14(\mathrm{~m}, 2 \mathrm{H}) . \mathrm{MS} m / z .515 .4(\mathrm{M}+$ $\mathrm{H}^{+}$.

\begin{abstract}
4-\{[[6-Cyano-1-(3-methyl-3H-imidazol-4-ylmethyl)-1,2,3,4-tetrahydro-quinolin-3-yl]-(3methyl-3H-imidazole-4-sulfonyl)-amino]-methyl\}-piperidine-1-carboxylic Acid Methyl Ester (191)

${ }^{1} \mathrm{H}$ NMR $\left(300 \mathrm{MHz}\right.$, methanol- $\left.d_{4}\right) \delta 8.93(\mathrm{~s} 1 \mathrm{H}), 7.76(\mathrm{~s}, 1 \mathrm{H}), 7.74(\mathrm{~s}, 1 \mathrm{H}), 7.45(\mathrm{~s}, 1 \mathrm{H}), 7.33$ $(\mathrm{dd}, J=1.8,8.4 \mathrm{~Hz}, 1 \mathrm{H}), 7.32(\mathrm{~s}, 1 \mathrm{H}), 6.82(\mathrm{~d}, J=8.7 \mathrm{~Hz}, 1 \mathrm{H}), 4.78(\mathrm{~d}, J=17.4 \mathrm{~Hz}, 1 \mathrm{H}), 4.70$ $(\mathrm{d}, J=17.4 \mathrm{~Hz}, 1 \mathrm{H}), 4.32-4.41(\mathrm{~m}, 1 \mathrm{H}), 3.99-4.1(\mathrm{~m}, 2 \mathrm{H}), 3.92(\mathrm{~s}, 3 \mathrm{H}), 3.80(\mathrm{~s}, 3 \mathrm{H}), 3.65(\mathrm{~s}$, $3 \mathrm{H}), 3.51-3.56(2 \mathrm{H}), 2.96-3.14(\mathrm{~m}, 4 \mathrm{H}), 2.71-2.78(\mathrm{~m}, 2 \mathrm{H}), 1.71-1.88(\mathrm{~m}, 3 \mathrm{H}), 1.00-1.13$ (m, 2H). MS $m / z 567.5\left(\mathrm{M}+\mathrm{H}^{+}\right)$.
\end{abstract}

4-(\{(Benzo[1,2,5]thiadiazole-4-sulfonyl)-[6-cyano-1-(3-methyl-3H-imidazol-4ylmethyl)-1,2,3,4-tetrahydro-quinolin-3-yl]-amino\}-methyl)-piperidine-1-carboxylic Acid Methyl Ester (188)

${ }^{1} \mathrm{H}$ NMR $\left(300 \mathrm{MHz}\right.$, methanol- $\left.d_{4}\right) \delta 8.94(\mathrm{~s} 1 \mathrm{H}), 8.32-8.35(\mathrm{~m}, 1 \mathrm{H}), 8.32(\mathrm{~s}, 1 \mathrm{H}), 7.08-7.85$ (m, 1H), $7.42(\mathrm{~s}, 1 \mathrm{H}), 7.35(\mathrm{dd}, J=1.8,8.7 \mathrm{~Hz}, 1 \mathrm{H}), 7.24(\mathrm{~s}, 1 \mathrm{H}), 6.82(\mathrm{~d}, J=8.7 \mathrm{~Hz}, 1 \mathrm{H})$, $4.78(\mathrm{~d}, J=18 \mathrm{~Hz}, 1 \mathrm{H}), 4.62(\mathrm{~d}, J=18 \mathrm{~Hz}, 1 \mathrm{H}), 4.04-4.08(\mathrm{~m}, 3 \mathrm{H}), 3.91(\mathrm{~s}, 3 \mathrm{H}), 3.66(\mathrm{~s}, 3 \mathrm{H})$, 3.49-3.51 (m, 3H), 3.03-3.10 (m, 2H), 2.61-2.73 (m, 3H), 1.71-1.88 (m, $3 \mathrm{H}), 1.02-1.14(\mathrm{~m}$, $2 \mathrm{H})$. MS $m / z 567.5\left(\mathrm{M}+\mathrm{H}^{+}\right)$.

4-\{[[6-Cyano-1-(3-methyl-3H-imidazol-4-ylmethyl)-1,2,3,4-tetrahydro-quinolin-3-yl]-(3,4dihydro-2H-benzo[b][1,4]dioxepine-7-sulfonyl)-amino]-methyl\}-piperidine-1-carboxylic Acid Methyl Ester (204)

${ }^{1} \mathrm{H}$ NMR $\left(300 \mathrm{MHz}\right.$, methanol- $\left.d_{4}\right) \delta 8.94(\mathrm{~s}, 1 \mathrm{H}), 7.45(\mathrm{~s}, 1 \mathrm{H}), 7.39(\mathrm{dd}, J=2.1,8.7 \mathrm{~Hz}, 1 \mathrm{H})$, $7.36(\mathrm{dd}, J=2.1,8.7 \mathrm{~Hz}, 1 \mathrm{H}), 7.35(\mathrm{~d}, J=2.1 \mathrm{~Hz}, 1 \mathrm{H}), 7.29(\mathrm{~s}, 1 \mathrm{H}), 7.25(\mathrm{~d}, J=8.7 \mathrm{~Hz}, 1 \mathrm{H})$, $7.10(\mathrm{~d}, J=8.7 \mathrm{~Hz}, 1 \mathrm{H}), 6.88(\mathrm{~d}, J=8.7 \mathrm{~Hz}, 1 \mathrm{H}), 4.64(\mathrm{~d}, J=16.7 \mathrm{~Hz}, 1 \mathrm{H}), 4.51(\mathrm{~d}, J=16.7$ $\mathrm{Hz}, 1 \mathrm{H}), 4.24-4.33(\mathrm{~m}, 4 \mathrm{H}), 4.04-4.14(\mathrm{~m}, 3 \mathrm{H}), 3.85(\mathrm{~s}, 3 \mathrm{H}), 3.64(\mathrm{~s}, 3 \mathrm{H}), 3.16-3.19(\mathrm{~m}, 1 \mathrm{H})$, 2.99-3.03 (m, 3H), 2.63-2.80 (m, 4H), 2.21-2.29 (m, 2H), 1.68-1.78 (m, 3H), 1.00-1.06 (m, $2 \mathrm{H})$. MS $m / z 635.7\left(\mathrm{M}+\mathrm{H}^{+}\right)$.

4-(\{(Benzo[1,2,5]oxadiazole-4-sulfonyl)-[6-cyano-1-(3-methyl-3H-imidazol-4ylmethyl)-1,2,3,4-tetrahydro-quinolin-3-yl]-amino\}-methyl)-piperidine-1-carboxylic Acid Methyl Ester (205)

${ }^{1} \mathrm{H}$ NMR $\left(300 \mathrm{MHz}\right.$, methanol- $\left.d_{4}\right) \delta 8.94(\mathrm{~s}, 1 \mathrm{H}), 8.27-8.29(\mathrm{~m}, 1 \mathrm{H}), 8.19-8.22(\mathrm{~m}, 1 \mathrm{H}), 7.68$ (s, 1H), 7.40-7.49 (m, 1H), 7.35 (dd, $J=1.8,8.7 \mathrm{~Hz}, 1 \mathrm{H}), 7.29(\mathrm{~s}, 1 \mathrm{H}), 6.82(\mathrm{~d}, J=8.7 \mathrm{~Hz}$, $1 \mathrm{H}), 4.78(\mathrm{~d}, J=16.7 \mathrm{~Hz}, 1 \mathrm{H}), 4.62(\mathrm{~d}, J=16.7 \mathrm{~Hz}, 1 \mathrm{H}), 4.04-4.08(\mathrm{~m}, 3 \mathrm{H}), 3.91(\mathrm{~s}, 3 \mathrm{H}), 3.66$ (s, 3H), 3.49-3.51 (m, 3H), 3.03-3.10 (m, 2H), 2.61-2.73 (m, 3H), 1.71-1.88 (m, 3H), 1.02$1.14(\mathrm{~m}, 2 \mathrm{H}) . \mathrm{MS} m / 2605.4\left(\mathrm{M}+\mathrm{H}^{+}\right)$.

4-\{[[6-Cyano-1-(3-methyl-3H-imidazol-4-ylmethyl)-1,2,3,4-tetrahydro-quinolin-3-yl]-(2oxo-2H-chromene-6-sulfonyl)-amino]-methyl\}-piperidine-1-carboxylic Acid Methyl Ester (206)

${ }^{1} \mathrm{H}$ NMR $\left(300 \mathrm{MHz}\right.$, methanol- $\left.d_{4}\right) \delta 8.93(\mathrm{~s}, 1 \mathrm{H}), 8.22(\mathrm{~d}, J=2.1 \mathrm{~Hz}, 1 \mathrm{H}), 8.07(\mathrm{dd}, J=2.1$, $8.7 \mathrm{~Hz}, 1 \mathrm{H}), 8.04(\mathrm{~d}, J=9.9 \mathrm{~Hz}, 1 \mathrm{H}), 7.53(\mathrm{~d}, J=8.7 \mathrm{~Hz}, 1 \mathrm{H}), 7.41(\mathrm{~d}, J=2.1 \mathrm{~Hz}, 1 \mathrm{H}), 7.37$ $(\mathrm{dd}, J=2.1,8.7 \mathrm{~Hz}, 1 \mathrm{H}), 7.26(\mathrm{~s}, 1 \mathrm{H}), 6.78(\mathrm{~d}, J=8.7 \mathrm{~Hz}, 1 \mathrm{H}), 6.58(\mathrm{~d}, J=9.9 \mathrm{~Hz}, 1 \mathrm{H}), 4.75$ $(\mathrm{d}, J=16.7 \mathrm{~Hz}, 1 \mathrm{H}), 4.68(\mathrm{~d}, J=16.7 \mathrm{~Hz}, 1 \mathrm{H}), 4.04-4.08(\mathrm{~m}, 3 \mathrm{H}), 3.90(\mathrm{~s}, 3 \mathrm{H}), 3.66(\mathrm{~s}, 3 \mathrm{H})$, 3.49-3.51 (m, 1H), 3.03-3.15 (m, 3H), 2.61-2.80 (m, 3H), 1.60-1.71 (m, 3H), 1.02-1.16 (m, $2 \mathrm{H})$. MS $m / z 631.4\left(\mathrm{M}+\mathrm{H}^{+}\right)$. 
4-(\{(4-Carboxy-furan-3-sulfonyl)-[6-cyano-1-(3-methyl-3H-imidazol-4-ylmethyl)-1,2,3,4tetrahydro-quinolin-3-yl]-amino\}-methyl)-piperidine-1-carboxylic Acid Methyl Ester (197)

${ }^{1} \mathrm{H}$ NMR $\left(300 \mathrm{MHz}\right.$, methanol- $\left.d_{4}\right) \delta 8.93(\mathrm{~s}, 1 \mathrm{H}), 7.43(\mathrm{dd}, J=2.1 \mathrm{~Hz}, 1 \mathrm{H}), 7.30(\mathrm{~d}, J=8.7$ $\mathrm{Hz}, 1 \mathrm{H}), 7.38(\mathrm{~d}, J=6.0 \mathrm{~Hz}, 1 \mathrm{H}), 7.36(\mathrm{~s}, 1 \mathrm{H}), 7.25(\mathrm{~d}, J=6.0 \mathrm{~Hz}, 1 \mathrm{H}), 6.83(\mathrm{~d}, J=8.7 \mathrm{~Hz}$, $1 \mathrm{H}), 6.88(\mathrm{~d}, J=8.7 \mathrm{~Hz}, 1 \mathrm{H}), 4.78(\mathrm{~d}, J=16.7 \mathrm{~Hz}, 1 \mathrm{H}), 4.62(\mathrm{~d}, J=16.7 \mathrm{~Hz}, 1 \mathrm{H}), 4.08-4.04$ $(\mathrm{m}, 3 \mathrm{H}), 3.91(\mathrm{~s}, 3 \mathrm{H}), 3.66(\mathrm{~s}, 3 \mathrm{H}), 3.49-3.51(\mathrm{~m}, 3 \mathrm{H}), 3.03-3.10(\mathrm{~m}, 2 \mathrm{H}), 2.61-2.73(\mathrm{~m}, 3 \mathrm{H})$, $1.71-1.88$ (m, 3H), 1.02-1.14 (m, 2H). MS m/z $597.4\left(\mathrm{M}+\mathrm{H}^{+}\right)$.

4-(\{[6-Cyano-1-(3-methyl-3H-imidazol-4-ylmethyl)-1,2,3,4-tetrahydro-quinolin-3-yl]methanesulfonylmethanesulfonyl-amino\}-methyl)-piperidine-1-carboxylic Acid Methyl Ester (207)

${ }^{1} \mathrm{H}$ NMR $\left(300 \mathrm{MHz}\right.$, methanol- $\left.d_{4}\right) \delta 8.96(\mathrm{~s}, 1 \mathrm{H}), 7.49(\mathrm{dd}, J=2.1,8.7 \mathrm{~Hz}, 1 \mathrm{H}), 7.47(\mathrm{~d}, J=$ $2.1 \mathrm{~Hz}, 1 \mathrm{H}), 6.88(\mathrm{~d}, J=8.7 \mathrm{~Hz}, 1 \mathrm{H}), 5.15(\mathrm{~s}, 2 \mathrm{H}), 4.68(\mathrm{~d}, J=16.7 \mathrm{~Hz}, 1 \mathrm{H}), 4.62(\mathrm{~d}, J=16.7$ $\mathrm{Hz}, 1 \mathrm{H}), 4.40-4.25(\mathrm{~m}, 1 \mathrm{H}), 4.04-4.14(\mathrm{~m}, 2 \mathrm{H}), 3.92(\mathrm{~s}, 3 \mathrm{H}), 3.73(\mathrm{~s}, 3 \mathrm{H}), 3.55-3.61(\mathrm{~m}, 3 \mathrm{H})$, $3.25(\mathrm{~s}, 3 \mathrm{H}), 3.16-3.19(\mathrm{~m}, 1 \mathrm{H}), 2.99-3.03(\mathrm{~m}, 2 \mathrm{H}), 2.63-2.80(\mathrm{~m}, 2 \mathrm{H}), 2.21-2.29(\mathrm{~m}, 1 \mathrm{H})$, $1.68-1.78(\mathrm{~m}, 2 \mathrm{H}), 1.05-1.16(\mathrm{~m}, 2 \mathrm{H}) . \mathrm{MS} \mathrm{m} / \mathrm{z} 579.2\left(\mathrm{M}+\mathrm{H}^{+}\right)$.

4-(\{[6-Cyano-1-(3-methyl-3H-imidazol-4-ylmethyl)-1,2,3,4-tetrahydro-quinolin-3-yl]cyclopropanesulfonyl-amino\}-methyl)-piperidine-1-carboxylic Acid Methyl Ester (202)

${ }^{1} \mathrm{H}$ NMR $\left(300 \mathrm{MHz}\right.$, methanol- $\left.d_{4}\right) \delta 8.94(\mathrm{~s}, 1 \mathrm{H}), 7.52(\mathrm{dd}, J=1.8,8.7 \mathrm{~Hz}, 1 \mathrm{H}), 7.48(\mathrm{~d}, J=$ $2.0 \mathrm{~Hz}), 7.42(\mathrm{~s}, 1 \mathrm{H}) 6.85(\mathrm{~d}, J=8.7 \mathrm{~Hz}, 1 \mathrm{H}), 4.78(\mathrm{~d}, J=16.7 \mathrm{~Hz}, 1 \mathrm{H}), 4.62(\mathrm{~d}, J=16.7 \mathrm{~Hz}$, $1 \mathrm{H}), 4.08-4.30(\mathrm{~m}, 3 \mathrm{H}), 3.95(\mathrm{~s}, 3 \mathrm{H}), 3.75(\mathrm{~s}, 3 \mathrm{H}), 3.51-3.72(\mathrm{~m}, 4 \mathrm{H}), 3.10-3.40(\mathrm{~m}, 4 \mathrm{H})$, 2.80-3.02 (m, 2H), 2.68-2.73 (m, 1H), 1.75-2.01 (m, 3H), 1.10-1.30 (brm, 6H). MS m/z 527.4 $\left(\mathrm{M}+\mathrm{H}^{+}\right)$.

3,4-Dihydro-2H-benzo[b][1,4]dioxepine-7-sulfonic Acid [6-Cyano-1-(3-methyl-3H-imidazol-4ylmethyl)-1,2,3,4-tetrahydro-quinolin-3-yl]-(4-methanesulfonyl-benzyl)-amide (223)

${ }^{1} \mathrm{H} \mathrm{NMR}\left(300 \mathrm{MHz}\right.$, methanol- $\left.d_{4}\right) \delta 8.94(\mathrm{~s}, 1 \mathrm{H}), 7.87(\mathrm{~d}, J=8.1 \mathrm{~Hz}, 2 \mathrm{H}), 7.62(\mathrm{~d}, J=8.1 \mathrm{~Hz}$, $2 \mathrm{H}), 7.45(\mathrm{~s}, 1 \mathrm{H}), 7.39(\mathrm{dd}, J=2.1,8.7 \mathrm{~Hz}, 1 \mathrm{H}), 7.36(\mathrm{dd}, J=2.1,8.7 \mathrm{~Hz}, 1 \mathrm{H}), 7.35(\mathrm{~d}, J=$ $2.1 \mathrm{~Hz}, 1 \mathrm{H}), 7.29(\mathrm{~s}, 1 \mathrm{H}), 7.25(\mathrm{~d}, J=8.7 \mathrm{~Hz}, 1 \mathrm{H}), 7.10(\mathrm{~d}, J=8.7 \mathrm{~Hz}, 1 \mathrm{H}), 6.68(\mathrm{~d}, J=8.7$ $\mathrm{Hz}, 1 \mathrm{H}), 4.64(\mathrm{~d}, J=16.7 \mathrm{~Hz}, 1 \mathrm{H}), 4.51(\mathrm{~d}, J=16.7 \mathrm{~Hz}, 1 \mathrm{H}), 4.24-4.33(\mathrm{~m}, 4 \mathrm{H}), 4.04-4.14$ (m, 2H), 3.85 (s, 3H), 3.20 (s, 3H), 2.95-3.05 (m, 1H), 1.12-1.25 (m, 2H). MS m/z 648.5 (M $+\mathrm{H}^{+}$).

3-Methyl-thiophene-2-sulfonic Acid [6-Cyano-1-(3-methyl-3H-imidazol-4-ylmethyl)-1,2,3,4tetrahydro-quinolin-3-yl]-(4-methanesulfonyl-benzyl)-amide (224)

${ }^{1} \mathrm{H}$ NMR $\left(300 \mathrm{MHz}\right.$, methanol- $\left.d_{4}\right) \delta 8.87(\mathrm{~s}, 1 \mathrm{H}), 7.98(\mathrm{~d}, J=8.1 \mathrm{~Hz}, 2 \mathrm{H}), 7.72(\mathrm{~d}, J=5.1 \mathrm{~Hz}$, $1 \mathrm{H}), 7.61(\mathrm{dd}, J=1.8,8.7 \mathrm{~Hz}, 1 \mathrm{H}), 7.35(\mathrm{~d}, J=8.7 \mathrm{~Hz}, 1 \mathrm{H}), 7.25(\mathrm{~m}, 2 \mathrm{H}), 7.07(\mathrm{~d}, J=5.1 \mathrm{~Hz}$, $1 \mathrm{H}), 6.70(\mathrm{~d}, J=8.7 \mathrm{~Hz}, 1 \mathrm{H}) 4.75(\mathrm{~d}, 2 \mathrm{H}), 4.53-4.55(\mathrm{~m}, 1 \mathrm{H}), 4.51(\mathrm{~d}, J=17.7 \mathrm{~Hz}, 1 \mathrm{H}), 4.48$ (d, $J=17.7 \mathrm{~Hz}, 1 \mathrm{H}), 3.79$ (s, 3H), 3.10-3.25 (m, 7H), 2.95-3.05 (m, 1H), $2.49(\mathrm{~s}, 3 \mathrm{H}) . \mathrm{MS} \mathrm{m} /$ $z 596.6\left(\mathrm{M}+\mathrm{H}^{+}\right)$.

$\mathrm{N}$-[6-Cyano-1-(3-methyl-3H-imidazol-4-ylmethyl)-1,2,3,4-tetrahydro-quinolin-3-yl]-2dimethylamino- $\mathrm{N}$-(2-fluoro-benzyl)-acetamide (248)

${ }^{1} \mathrm{H}$ NMR $\left(300 \mathrm{MHz}\right.$, methanol- $\left.d_{4}\right) \delta 8.78(\mathrm{~s}, 1 \mathrm{H}), 7.01-7.34(\mathrm{~m}, 7 \mathrm{H}), 6.68(\mathrm{~d}, J=8.7 \mathrm{~Hz}, 1 \mathrm{H})$, $4.42-4.61(\mathrm{~m}, 3 \mathrm{H}), 4.25(\mathrm{~d}, J=15.9 \mathrm{~Hz}, 1 \mathrm{H}), 4.18(\mathrm{~d}, J=15.9 \mathrm{~Hz}, 1 \mathrm{H}), 3.74(\mathrm{~s}, 3 \mathrm{H}), 3.41-$ $3.59(\mathrm{~m}, 2 \mathrm{H}), 3.19(\mathrm{~s}, 2 \mathrm{H}), 2.94-3.04(\mathrm{~m}, 1 \mathrm{H}), 2.86(\mathrm{~m}, 7 \mathrm{H})$. MS m/z $461.3\left(\mathrm{M}+\mathrm{H}^{+}\right)$. 
$\mathrm{N}$-[6-Cyano-1-(3-methyl-3H-imidazol-4-ylmethyl)-1,2,3,4-tetrahydro-quinolin-3-yl]- $\mathrm{N}$-(2fluoro-benzyl)-3-methylsulfanyl-propionamide (247)

${ }^{1} \mathrm{H}$ NMR $\left(300 \mathrm{MHz}\right.$, methanol- $\left.d_{4}\right) \delta 8.84(\mathrm{~s}, 1 \mathrm{H}), 7.01-7.34(\mathrm{~m}, 7 \mathrm{H}), 6.78(\mathrm{~d}, J=7.6 \mathrm{~Hz}, 1 \mathrm{H})$, 4.42-4.77 (m, 5H), 3.84 (s, 3H), 3.41-3.59 (m, 2H), 2.94-3.12 (m, 6H), 2.06 (s, 3H). MS m/ z $478.4\left(\mathrm{M}+\mathrm{H}^{+}\right)$.

General Procedure for the Synthesis of Compounds According to Scheme 4. 1-Methyl-1Himidazole-4-sulfonic Acid [6-Cyano-1-(1-trityl-1 H-imidazol-4-ylmethyl)-1,2,3,4-tetrahydroquinolin-3-yl]-amide (15)

A solution of $14(0.397 \mathrm{~g}, 1 \mathrm{mmol}), N, N$-diisopropylethyl amine $(2.37 \mathrm{~mL}, 2 \mathrm{mmol})$, and $3 \mathrm{~mL}$ of dry DMF was stirred at room temperature for $30 \mathrm{~min}$. To this solution was added dropwise a solution of triphenylmethyl chloride $(0.278 \mathrm{~g}, 1 \mathrm{mmol})$ dissolved in dry DMF $(3 \mathrm{~mL})$. The reaction mixture was stirred at room temperature for $20 \mathrm{~h}$. After addition of water $(10 \mathrm{~mL})$, the solution was extracted with dichloromethane $(3 \times 15 \mathrm{~mL})$. The combined organic layer was washed with brine $(20 \mathrm{~mL})$ and the organic layer was dried over $\mathrm{MgSO}_{4}$ and evaporated under reduce pressure, yielding the crude product, which was purified on a silica gel column eluting with $10 \%$ methanol in ethyl acetate to afford $15(0.562 \mathrm{~g}, 88 \%) .{ }^{1} \mathrm{H}$ NMR $(300 \mathrm{MHz}$, $\left.\mathrm{CDCl}_{3}\right) \delta 8.0(\mathrm{~s}, 1 \mathrm{H}), 7.9(\mathrm{~s}, 1 \mathrm{H}), 7.4(\mathrm{~s}, 1 \mathrm{H}), 7.25-7.37(\mathrm{~m}, 18 \mathrm{H}), 6.48(\mathrm{~d}, J=8.7 \mathrm{~Hz}, 1 \mathrm{H})$, $4.5(\mathrm{~d}, J=16.2 \mathrm{~Hz}, 1 \mathrm{H}), 4.23(\mathrm{~d}, J=16.2 \mathrm{~Hz}, 1 \mathrm{H}), 4.00-4.09(\mathrm{~m}, 1 \mathrm{H}), 3.70(\mathrm{~s}, 3 \mathrm{H}), 3.51-3.61$ (m, 1H), 3.31-3.34 (m, 1H), 3.09-3.12 (m, 1H), 2.86-3.07 (m, 1H). MS m/z $640.3\left(\mathrm{M}+\mathrm{H}^{+}\right)$.

A solution of $16(0.21 \mathrm{mmol})$, dichloromethane $(3 \mathrm{~mL})$, and trifluoroacetic acid $(0.5 \mathrm{~mL})$ was stirred for $30 \mathrm{~min}$. The reaction mixture was filtered to remove trityl alcohol, and the residue was washed with dichloromethane. The filtrate and washings were combined and then evaporated to dryness via rotary evaporation. The residue was purified by HPLC.

\section{1-Methyl-1H-imidazole-4-sulfonic Acid Benzyl-[6-cyano-1-(3H-imidazol-4-ylmethyl)-1,2,3,4- tetrahydro-quinolin-3-yl]-amide (253)}

${ }^{1} \mathrm{H}$ NMR $\left(300 \mathrm{MHz}\right.$, methanol- $\left.d_{4}\right) \delta 8.86(\mathrm{~s}, 1 \mathrm{H}), 7.83(\mathrm{~s}, 1 \mathrm{H}), 7.71(\mathrm{~s}, 1 \mathrm{H}), 7.18-7.39(\mathrm{~m}, 8$ $\mathrm{H}), 6.71(\mathrm{~d}, J=9.7 \mathrm{~Hz}, 1 \mathrm{H}), 4.29-4.73(\mathrm{~m}, 5 \mathrm{H}), 3.80(\mathrm{~s}, 3 \mathrm{H}), 3.36-3.42(\mathrm{~m}, 1 \mathrm{H}), 3.18-3.26$ (m, 1H), 3.12 (dd, $J=8.7,15.6 \mathrm{~Hz}, 1 \mathrm{H}), 2.89$ (dd, $J=3.3,15.3 \mathrm{~Hz}, 1 \mathrm{H})$. MS $\mathrm{m} / z 488.1(\mathrm{M}+$ $\left.\mathrm{H}^{+}\right)$.

\section{1-Methyl-1 H-imidazole-4-sulfonic Acid (2-Bromo-allyl)-[6-cyano-1-(3H-imidazol-4- ylmethyl)-1,2,3,4-tetrahydro-quinolin-3-yl]-amide (252)}

${ }^{1} \mathrm{H}$ NMR $\left(300 \mathrm{MHz}\right.$, methanol- $\left.d_{4}\right) \delta 8.86(\mathrm{~s}, 1 \mathrm{H}), 7.83(\mathrm{~s}, 1 \mathrm{H}), 7.79(\mathrm{~s}, 1 \mathrm{H}), 7.28(\mathrm{~s}, 1 \mathrm{H}), 7.33$ $(\mathrm{dd}, J=2.1,9.0 \mathrm{~Hz}, 1 \mathrm{H}), 7.28(\mathrm{~s}, 1 \mathrm{H}), 6.65(\mathrm{~d}, J=8.7 \mathrm{~Hz}, 1 \mathrm{H}), 6.04(\mathrm{~s}, 1 \mathrm{H}), 5.65(\mathrm{~s}, 1 \mathrm{H}), 4.79$ $(\mathrm{d}, J=17.1 \mathrm{~Hz}, 1 \mathrm{H}), 4.62(\mathrm{~d}, J=17.1 \mathrm{~Hz}, 1 \mathrm{H}), 4.39-4.59(\mathrm{~m}, 1 \mathrm{H}), 4.19(\mathrm{~d}, J=17.1 \mathrm{~Hz}, 1 \mathrm{H})$ $4.0(\mathrm{~d}, J=17.1 \mathrm{~Hz}, 1 \mathrm{H}), 3.81(\mathrm{~s}, 3 \mathrm{H}), 3.55-3.62(\mathrm{~m}, 1 \mathrm{H}), 3.42-3.47(\mathrm{~m}, 1 \mathrm{H}), 3.07-3.14(\mathrm{~m}$, $1 \mathrm{H}), 2.97(\mathrm{dd}, J=4.5,15.9 \mathrm{~Hz}, 1 \mathrm{H})$. MS $m / z 518.2\left(\mathrm{M}+\mathrm{H}^{+}\right)$.

\section{Synthesis of 255 According to Scheme 5. 4-\{[(6-cyano-1,2,3,4-tetrahydro-quinolin-3-yl)- (pyridine-2-sulfonyl)-amino]-methyl\}-piperidine-1-carboxylic Acid Methyl Ester (18)}

${ }^{1} \mathrm{H}$ NMR $\left(300 \mathrm{MHz}\right.$, methanol- $\left.d_{4}\right) \delta 8.70(\mathrm{~d}, J=7.5 \mathrm{~Hz}, 1 \mathrm{H}), 8.08(\mathrm{td}, J=1.8,7.8 \mathrm{~Hz}, 1 \mathrm{H})$, $8.0(\mathrm{dt}, J=1.2,7.8 \mathrm{~Hz}, 1 \mathrm{H}), 7.66(\mathrm{ddd}, J=1.5,4.8,7.5 \mathrm{~Hz}, 1 \mathrm{H}), 7.43(\mathrm{~d}, J=1.2 \mathrm{~Hz}, 1 \mathrm{H}), 7.38$ $(\mathrm{dd}, J=2.1,8.7 \mathrm{~Hz}, 1 \mathrm{H}), 6.81(\mathrm{~d}, J=8.7 \mathrm{~Hz}, 1 \mathrm{H}), 4.38-4.51(\mathrm{~m}, 1 \mathrm{H}), 4.08-4.16(\mathrm{~m}, 2 \mathrm{H}), 3.65$ (s, 3H), 3.51-3.62 (m, 2H), 3.21-3.26 (m, 2H), 3.11-3.18 (m, 1H), 2.79-2.87 (m, 3H), 1.68$1.88,(\mathrm{~m}, 3 \mathrm{H}), 1.05-1.17(\mathrm{~m}, 2 \mathrm{H})$. MS m/z $470.2\left(\mathrm{M}+\mathrm{H}^{+}\right)$. 


\section{4-\{[[6-Cyano-1-(3-methyl-3H-imidazole-4-sulfonyl)-1,2,3,4-tetrahydro-quinolin-3-yl]- (pyridine-2-sulfonyl)-amino]-methyl\}-piperidine-1-carboxylic Acid Methyl Ester (255)}

A solution of 18 (47 mg, $0.1 \mathrm{mmol}$ ), 3-methyl-3H-imidazole-4-sulfonyl chloride hydrochloride salt (26 mg, $0.12 \mathrm{mmol}$, Apollo Scientific Intermediates), $N, N$-dimethyl pyridine ( $15 \mathrm{mg}, 0.12$ $\mathrm{mmol}$ ), and $3 \mathrm{~mL}$ of dry acetonitrile was refluxed overnight under nitrogen. Then the reaction mixture was quenched with water and extracted with dichloromethane $(3 \times 10 \mathrm{~mL})$. The combine organic layer was washed with brine $(10 \mathrm{~mL})$, and the organic layer was dried over $\mathrm{MgSO}_{4}$ and evaporated under reduced pressure, yielding the crude product, which was purified by HPLC. ${ }^{1} \mathrm{H}$ NMR $\left(300 \mathrm{MHz}\right.$, methanol- $\left.d_{4}\right) \delta 8.76(\mathrm{~d}, J=4.8 \mathrm{~Hz}, 1 \mathrm{H}), 8.08(\mathrm{td}, J=1.8,8.1$ $\mathrm{Hz}, 1 \mathrm{H}), 8.0(\mathrm{dt}, J=1.2,7.8 \mathrm{~Hz}, 1 \mathrm{H}), 7.89(\mathrm{~d}, J=8.4 \mathrm{~Hz}, 1 \mathrm{H}), 7.81(\mathrm{~s}, 1 \mathrm{H}), 7.66(\mathrm{~m}, 2 \mathrm{H}), 7.48$ $(\mathrm{dd}, J=2.1,8.7 \mathrm{~Hz}, 1 \mathrm{H}), 7.43(\mathrm{~s}, 1 \mathrm{H}), 4.32-4.38(\mathrm{~m}, 1 \mathrm{H}), 4.14-4.25(\mathrm{~m}, 1 \mathrm{H}), 4.09-4.13(\mathrm{~m}$, $2 \mathrm{H}), 3.72(\mathrm{~s}, 3 \mathrm{H}), 3.68(\mathrm{~s}, 3 \mathrm{H}), 3.51-3.62(\mathrm{~m}, 1 \mathrm{H}), 3.21-3.26(\mathrm{~m}, 2 \mathrm{H}), 3.11-3.18(\mathrm{~m}, 1 \mathrm{H})$, 2.79-2.87 (m, 3H), 1.92-1.99 (m, 1H), 1.68-1.88 (m, 2H), 1.05-1.17 (m, 2H). MS m/z 614.4 $\left(\mathrm{M}+\mathrm{H}^{+}\right)$.

\section{[[1-(3-Benzyl-3H-imidazol-4-ylmethyl)-6-cyano-1,2,3,4-tetrahydro-quinolin-3-yl]-(1- methyl-1 $\mathrm{H}$-imidazole-4-sulfonyl)-amino]-acetic Acid tert-Butyl Ester (254)}

${ }^{1} \mathrm{H}$ NMR $\left(300 \mathrm{MHz}\right.$, methanol- $\left.\left.d_{4}\right) \delta 8.86(\mathrm{~s}, 1 \mathrm{H}), 7.11-7.50(\mathrm{~m}, 10 \mathrm{H}), 6.55(\mathrm{~d}, J) 8.5 \mathrm{~Hz}, 1 \mathrm{H}\right)$, $5.12(\mathrm{~s}, 2 \mathrm{H}), 4.62-4.78(\mathrm{~m}, 2 \mathrm{H}), 4.39-4.59(\mathrm{~m}, 1 \mathrm{H}), 4.19(\mathrm{~d}, J) 17.1 \mathrm{~Hz}, 1 \mathrm{H}) 4.0(\mathrm{~d}, J) 17.1$ $\mathrm{Hz}, 1 \mathrm{H}), 3.81(\mathrm{~s}, 3 \mathrm{H}), 3.55-3.62(\mathrm{~m}, 1 \mathrm{H}), 3.42-3.47(\mathrm{~m}, 1 \mathrm{H}), 3.07-3.14(\mathrm{~m}, 1 \mathrm{H}), 2.97(\mathrm{~m}, 1 \mathrm{H})$, $1.42(\mathrm{~s}, 9 \mathrm{H}) . \mathrm{MS} m / z 602.2\left(\mathrm{M}+\mathrm{H}^{+}\right)$.

\section{4-\{[(6-Cyano-1-pyridin-3-ylmethyl-1,2,3,4-tetrahydro-quinolin-3-yl)-(pyridine-2-sulfonyl)- amino]-methyl\}-piperidine-1-carboxylic Acid Methyl Ester (256)}

${ }^{1} \mathrm{H}$ NMR (300 MHz, methanol- $\left.d_{4}\right) \delta 8.84-8.89(\mathrm{~m}, 2 \mathrm{H}), 8.70-8.75(\mathrm{~m}, 1 \mathrm{H}), 8.49-8.51(\mathrm{~m}$, $1 \mathrm{H}), 8.03-8.19(\mathrm{~m}, 3 \mathrm{H}), 7.68$ (ddd, $J=1.5,4.8,7.5 \mathrm{~Hz}, 1 \mathrm{H}), 7.43(\mathrm{~d}, J=1.2 \mathrm{~Hz}, 1 \mathrm{H}), 7.38$ $(\mathrm{dd}, J=2.1,8.7 \mathrm{~Hz}, 1 \mathrm{H}), 6.81(\mathrm{~d}, J=8.7 \mathrm{~Hz}, 1 \mathrm{H}), 4.68-4.83(\mathrm{~m}, 2 \mathrm{H}), 4.38-4.51(\mathrm{~m}, 1 \mathrm{H})$, $4.08-4.16(\mathrm{~m}, 2 \mathrm{H}), 3.65(\mathrm{~s}, 3 \mathrm{H}), 3.51-3.62(\mathrm{~m}, 2 \mathrm{H}), 3.21-3.26(\mathrm{~m}, 2 \mathrm{H}), 3.11-3.18(\mathrm{~m}, 1 \mathrm{H})$, 2.79-2.87 (m, 3H), 1.68-1.88, (m, 3H), 1.05-1.17 (m, 2H). MS m/z $561.4\left(\mathrm{M}+\mathrm{H}^{+}\right)$.

\section{General Procedure for the Synthesis of Compounds According to Scheme 6}

A suspension of the aryl bromide 21 (500 mg, $1.53 \mathrm{mmol}$ ), phenyl boronic acid (559 mg, 4.58 $\mathrm{mmol})$, and tetrakis triphenylphosphine palladium (177 $\mathrm{mg}, 10 \mathrm{~mol} \%)$ in dimethoxyethane $(15 \mathrm{~mL})$ was thoroughly degassed and stirred under argon. Deionized water $(3 \mathrm{~mL})$ and barium hydroxide octahydrate $(1.45 \mathrm{gm}, 4.58 \mathrm{mmol})$ were added, and the reaction mixture was heated at reflux for $2 \mathrm{~h}$. The reaction mixture was diluted with ethyl acetate, washed with water and brine, dried $\left(\mathrm{Na}_{2} \mathrm{SO}_{4}\right)$, and concentrated under reduced pressure. The residue was purified by flash chromatography over a silica gel column to give $\mathbf{2 2}(120 \mathrm{mg}, 45 \%)$ as a colorless solid. ${ }^{1} \mathrm{H}$ NMR $\left(\mathrm{CDCl}_{3}\right) \delta 7.49(\mathrm{~d}, J=8.1 \mathrm{~Hz}, 1 \mathrm{H}), 7.36(\mathrm{t}, J=7.8 \mathrm{~Hz}, 2 \mathrm{H}), 7.23(\mathrm{t}, J=7.4$ $\mathrm{Hz}, 4 \mathrm{H}), 6.51(\mathrm{~d}, J=8.1 \mathrm{~Hz}, 1 \mathrm{H}), 5.04(\mathrm{~d}, J=7.5 \mathrm{~Hz}, 1 \mathrm{H}), 4.11(\mathrm{br} \mathrm{s}, 1 \mathrm{H}), 3.95(\mathrm{~s}, 1 \mathrm{H}), 3.37-$ $3.33(\mathrm{~m}, 1 \mathrm{H}), 3.19-3.16(\mathrm{~m}, 1 \mathrm{H}), 3.09-3.02(\mathrm{~m}, 1 \mathrm{H}), 2.77-2.72(\mathrm{~m}, 1 \mathrm{H}), 1.43(\mathrm{~s}, 9 \mathrm{H})$.

To a stirred solution of the phenyl derivative $22(120 \mathrm{mg}, 0.37 \mathrm{mmol})$ in $\mathrm{CH}_{2} \mathrm{Cl}_{2}(1.6 \mathrm{~mL})$ at room temperature, trifluoroacetic acid $(0.4 \mathrm{~mL})$ was added, and the reaction mixture was stirred for $2 \mathrm{~h}$. The solution was concentrated under reduced pressure, and the crude amine (trifluoroacetate salt) $\mathbf{2 3}$ was taken to the next step without further purification.

To a mixture of the amine 23 in $\mathrm{CH}_{2} \mathrm{Cl}_{2}(2 \mathrm{~mL})$, diisopropylethylamine $(0.5 \mathrm{~mL})$ was added and the reaction mixture was stirred at room temperature for $0.5 \mathrm{~h}$. The appropriate sulfonyl chloride $(0.37 \mathrm{mmol})$ was added, and the reaction mixture was stirred for an additional $2 \mathrm{~h}$. The reaction mixture was partitioned between water and ethyl acetate. The organic layer was 
washed with brine, dried $\left(\mathrm{Na}_{2} \mathrm{SO}_{4}\right)$, and evaporated under reduced pressure. Purification by flash chromatography gave $\mathbf{2 4}(89 \%)$ as a pale yellow solid. ${ }^{1} \mathrm{H}$ NMR (methanol- $\left.d_{4}\right) \delta 7.93$ $(\mathrm{s}, 1 \mathrm{H}), 7.76(\mathrm{~s}, 1 \mathrm{H}), 7.69(\mathrm{~d}, J=1.2 \mathrm{~Hz}, 1 \mathrm{H}), 7.49(\mathrm{dd}, J=1.2,8.4 \mathrm{~Hz}, 2 \mathrm{H}), 7.35(\mathrm{t}, J=7.5$ $\mathrm{Hz}, 2 \mathrm{H}), 7.22(\mathrm{~d}, J=7.5 \mathrm{~Hz}, 1 \mathrm{H}), 7.11(\mathrm{~s}, 1 \mathrm{H}), 6.60(\mathrm{~d}, J=8.4 \mathrm{~Hz}, 1 \mathrm{H}), 3.77(\mathrm{~s}, 3 \mathrm{H}), 3.74-$ 3.69 (m, 1H), 3.39-3.36 (m, 1H), 3.09 (dd, $J=8.1,11.4 \mathrm{~Hz}, 1 \mathrm{H}), 2.93(\mathrm{dd}, J=4.5,15.6 \mathrm{~Hz}$, $1 \mathrm{H}), 2.76(\mathrm{dd}, J=8.1,15.6 \mathrm{~Hz}, 1 \mathrm{H})$.

To a stirred solution of the sulfonamide $24(0.33 \mathrm{mmol})$ in dichloroethane $(1 \mathrm{~mL})$ and trifluoroacetic acid $(1 \mathrm{~mL})$ was added 3-methyl-3H-imidazole-4-carbaldehyde $(91 \mathrm{mg}, 0.83$ $\mathrm{mmol}$ ) at room temperature, and the reaction mixture was heated to $50^{\circ} \mathrm{C}$ for $1 \mathrm{~h}$. Triethylsilane $(0.13 \mathrm{~mL}, 0.83 \mathrm{mmol})$ was added and stirring was continued for an additional $48 \mathrm{~h}$. Volatile materials were removed under vacuum, and the reaction mixture was diluted with ethyl acetate, washed with aqueous $\mathrm{NaHCO}_{3}$ solution, water, and brine, dried $\left(\mathrm{Na}_{2} \mathrm{SO}_{4}\right)$, filtered, and concentrated in vacuum. Purification by flash chromatography afforded $\mathbf{2 5}(50 \mathrm{mg}, 33 \%)$ as a pale yellow foam. ${ }^{1} \mathrm{H}$ NMR (methanol- $\left.d_{4}\right) \delta 8.89(\mathrm{~s}, 1 \mathrm{H}), 8.06(\mathrm{~s}, 1 \mathrm{H}), 7.67(\mathrm{~s}, 1 \mathrm{H}), 7.49(\mathrm{t}$, $J=7.8 \mathrm{~Hz}, 3 \mathrm{H}), 7.37-7.33(\mathrm{~m}, 3 \mathrm{H}), 7.24-7.20(\mathrm{~m}, 2 \mathrm{H}), 6.85(\mathrm{~d}, J=7.8 \mathrm{~Hz}, 1 \mathrm{H}), 4.74(\mathrm{~d}, J=$ $17.1 \mathrm{~Hz}, 1 \mathrm{H}), 4.57(\mathrm{~d}, J=17.1 \mathrm{~Hz}, 1 \mathrm{H}), 3.93-3.71(\mathrm{~m}, 8 \mathrm{H}), 3.35-3.31(\mathrm{~m}, 1 \mathrm{H}), 3.04(\mathrm{dd}, J=$ $4.5,15.6 \mathrm{~Hz}, 1 \mathrm{H}), 2.76(\mathrm{dd}, J=6.3,15.9 \mathrm{~Hz}, 1 \mathrm{H})$.

A mixture of 25 ( 1 equiv), $\mathrm{Cs}_{2} \mathrm{CO}_{3}$ ( 2 equiv), and the appropriate bromide (1 equiv) in anhydrous DMF was stirred overnight at room temperature. The reaction mixture was partitioned between water and ethyl acetate. The organic layer was washed with brine, dried $\left(\mathrm{Na}_{2} \mathrm{SO}_{4}\right)$, and evaporated under reduced pressure. The crude residue was purified by HPLC.

\section{1-Methyl-1 H-imidazole-4-sulfonic Acid [1-(3-Methyl-3H-imi-dazol-4-ylmethyl)-6- phenyl-1,2,3,4-tetrahydro-quinolin-3-yl]-(2-pyrrol-1-yl-ethyl)-amide (272)}

${ }^{1} \mathrm{H}$ NMR (methanol- $\left.d_{4}\right) \delta 7.74(\mathrm{~d}, J=1.0 \mathrm{~Hz}, 1 \mathrm{H}), 7.70(\mathrm{~d}, J=1.5 \mathrm{~Hz}, 1 \mathrm{H}), 7.53$ (br s, $\left.1 \mathrm{H}\right)$, 7.52-7.50 (m, 2H), 7.39-7.33 (m, 3H), 7.26-7.22 (m, 1H), 7.17-7.16 (m, 1H), $6.90(\mathrm{~d}, J=8.5$ $\mathrm{Hz}, 1 \mathrm{H}), 6.80(\mathrm{br} \mathrm{s}, 1 \mathrm{H}), 6.63(\mathrm{t}, J=2.0 \mathrm{~Hz}, 2 \mathrm{H}), 6.04(\mathrm{t}, J=2.0 \mathrm{~Hz}, 2 \mathrm{H}), 4.44(\mathrm{~d}, J=15.5 \mathrm{~Hz}$, $1 \mathrm{H}), 4.27(\mathrm{~d}, J=15.5 \mathrm{~Hz}, 1 \mathrm{H}), 4.22-4.17(\mathrm{~m}, 4 \mathrm{H}), 3.75(\mathrm{~s}, 3 \mathrm{H}), 3.63(\mathrm{~s}, 3 \mathrm{H}), 3.48-3.44(\mathrm{~m}$, 2H), $2.91(\mathrm{dd}, J=4.5,11.5 \mathrm{~Hz}, 1 \mathrm{H}), 2.76-2.66(\mathrm{~m}, 2 \mathrm{H}) . \mathrm{MS} m / z 556.3\left(\mathrm{M}+\mathrm{H}^{+}\right)$.

\section{N-tert-Butyl-2-\{(1-methyl-1 $\mathrm{H}$-imidazole-4-sulfonyl)-[1-(3-methyl-3H-imidazol-4-ylmethyl)-6- phenyl-1,2,3,4-tetrahydro-quinolin-3-yl]-amino\}-acetamide (267)}

${ }^{1} \mathrm{H}$ NMR (methanol- $\left.d_{4}\right) \delta 8.87(\mathrm{~s}, 1 \mathrm{H}), 7.78(\mathrm{~d}, J=1.2 \mathrm{~Hz}, 2 \mathrm{H}), 7.45(\mathrm{~d}, J=7.2 \mathrm{~Hz}, 2 \mathrm{H}), 7.41$ $(\mathrm{s}, 1 \mathrm{H}), 7.32(t, J=7.5 \mathrm{~Hz}, 3 \mathrm{H}), 7.19(t, J=7.2 \mathrm{~Hz}, 2 \mathrm{H}), 6.82(\mathrm{~d}, J=7.8 \mathrm{~Hz}, 1 \mathrm{H}), 4.65(d, J=$ $15.6 \mathrm{~Hz}, 1 \mathrm{H}), 4.52(\mathrm{~d}, J=15.6 \mathrm{~Hz}, 1 \mathrm{H}), 4.36-4.18(\mathrm{~m}, 1 \mathrm{H}), 3.94-3.75(\mathrm{~m}, 9 \mathrm{H}), 3.31-3.40(\mathrm{~m}$, $1 \mathrm{H}), 3.02-2.85(\mathrm{~m}, 2 \mathrm{H}), 1.31(\mathrm{~m}, 9 \mathrm{H})$. MS m/z $576.4\left(\mathrm{M}+\mathrm{H}^{+}\right)$.

\section{1-Methyl-1 H-imidazole-4-sulfonic Acid (4-Methanesulfonyl-benzyl)-[1-(3-methyl-3H- imidazol-4-ylmethyl)-6-phenyl-1,2,3,4-tetrahydroquinolin-3-yl]-amide (278)}

${ }^{1} \mathrm{H}$ NMR $\left(300 \mathrm{MHz}\right.$, methanol- $\left.d_{4}\right) \delta 8.87(\mathrm{~s}, 1 \mathrm{H}), 7.92(\mathrm{~d}, J=8.4 \mathrm{~Hz}, 2 \mathrm{H}), 7.85(\mathrm{~s}, 1 \mathrm{H}), 7.80$ (s, 1H), $7.62(\mathrm{~d}, J=8.4 \mathrm{~Hz}, 2 \mathrm{H}), 7.52(\mathrm{~d}, J=8.4 \mathrm{~Hz}, 2 \mathrm{H}), 7.40-7.30(\mathrm{~m}, 4 \mathrm{H}), 7.28-7.22(\mathrm{~m}$, $2 \mathrm{H}), 6.72(\mathrm{~d}, J=8.4 \mathrm{~Hz}, 1 \mathrm{H}), 4.75(\mathrm{~d}, J=16.5 \mathrm{~Hz}, 2 \mathrm{H}), 4.55-4.50(\mathrm{~m}, 1 \mathrm{H}), 4.48(\mathrm{~d}, J=17.4$ $\mathrm{Hz}, 1 \mathrm{H}), 4.35(\mathrm{~d}, J=16.5 \mathrm{~Hz}, 1 \mathrm{H}), 3.85(\mathrm{~s}, 3 \mathrm{H}), 3.80(\mathrm{~s}, 3 \mathrm{H}), 3.42-3.36(\mathrm{~m}, 2 \mathrm{H}), 3.12(\mathrm{~s}, 3 \mathrm{H})$, 3.08-2.95 (m, 2H). MS m/z $631.6\left(\mathrm{M}+\mathrm{H}^{+}\right)$.

\section{1-Methyl-1H-imidazole-4-sulfonic Acid (2-Bromo-allyl)-[1-(3-methyl-3H-imidazol-4- ylmethyl)-6-phenyl-1,2,3,4-tetrahydro-quinolin-3-yl]-amide (264)}

${ }^{1} \mathrm{H}$ NMR $\left(300 \mathrm{MHz}\right.$, methanol- $\left.d_{4}\right) \delta 8.92(\mathrm{~s}, 1 \mathrm{H}), 7.80(\mathrm{~d}, J=3.9 \mathrm{~Hz}, 2 \mathrm{H}), 7.55(\mathrm{dd}, J=1.2$, $8.4 \mathrm{~Hz}, 2 \mathrm{H}), 7.45(\mathrm{~s}, 1 \mathrm{H}), 7.40(\mathrm{t}, J=8.7 \mathrm{~Hz}, 3 \mathrm{H}), 7.32-7.20(\mathrm{~m}, 2 \mathrm{H}), 6.90(\mathrm{~d}, J=8.7 \mathrm{~Hz}, 1 \mathrm{H})$, 
$6.10(\mathrm{~d}, J=2.1 \mathrm{~Hz}, 1 \mathrm{H}), 5.65(\mathrm{~d}, J=2.1 \mathrm{~Hz}, 1 \mathrm{H}), 4.75(\mathrm{~d}, J=16.8 \mathrm{~Hz}, 1 \mathrm{H}), 4.60(\mathrm{~d}, J=17.1$ $\mathrm{Hz}, 1 \mathrm{H}), 4.50-4.40(\mathrm{~m}, 1 \mathrm{H}), 4.20(\mathrm{~d}, J=18.0 \mathrm{~Hz}, 1 \mathrm{H}), 4.10(\mathrm{~d}, J=18.0 \mathrm{~Hz}, 1 \mathrm{H}), 3.95(\mathrm{~s}, 3 \mathrm{H})$, $3.80(\mathrm{~s}, 3 \mathrm{H}), 3.49-3.40(\mathrm{~m}, 2 \mathrm{H}), 3.15-3.05(\mathrm{~m}, 1 \mathrm{H}), 2.87(\mathrm{dd},=5.1,15.6 \mathrm{~Hz}, 1 \mathrm{H}) . \mathrm{MS} m / z$ $583.0\left(\mathrm{M}+\mathrm{H}^{+}\right)$.

1-Methyl-1H-imidazole-4-sulfonic Acid Ethyl-[1-(3-methyl-3H-imidazol-4-ylmethyl)-6phenyl-1,2,3,4-tetrahydro-quinolin-3-yl]amide (259)

${ }^{1} \mathrm{H}$ NMR $\left(300 \mathrm{MHz}\right.$, methanol- $\left.d_{4}\right) \delta 8.90(\mathrm{~s}, 1 \mathrm{H}), 7.75(\mathrm{~s}, 1 \mathrm{H}), 7.72(\mathrm{~s}, 1 \mathrm{H}), 7.52(\mathrm{dd}, J=1.2$, $8.7 \mathrm{~Hz}, 2 \mathrm{H}), 7.45(\mathrm{~s}, 1 \mathrm{H}), 7.35(\mathrm{t}, J=7.2 \mathrm{~Hz}, 3 \mathrm{H}), 7.30-7.20(\mathrm{~m}, 2 \mathrm{H}), 6.87(\mathrm{~d}, J=8.7 \mathrm{~Hz}, 1 \mathrm{H})$, $4.75(\mathrm{~d}, J=16.8 \mathrm{~Hz}, 1 \mathrm{H}), 4.57(\mathrm{~d}, J=16.8 \mathrm{~Hz}, 1 \mathrm{H}), 4.47-4.37(\mathrm{~m}, 1 \mathrm{H}), 3.95(\mathrm{~s}, 3 \mathrm{H}), 3.80(\mathrm{~s}$, $3 \mathrm{H}), 3.45-3.25(\mathrm{~m}, 4 \mathrm{H}), 3.20-3.10(\mathrm{~m}, 1 \mathrm{H}), 2.90(\mathrm{~m}, 1 \mathrm{H}), 1.25(\mathrm{t}, J=6.9 \mathrm{~Hz}, 3 \mathrm{H}) . \mathrm{MS} m / z$ $491.5\left(\mathrm{M}+\mathrm{H}^{+}\right)$.

\{(1-Methyl-1 H-imidazole-4-sulfonyl)-[1-(3-methyl-3H-imidazol-4-ylmethyl)-6-phenyl-1,2,3,4tetrahydro-quinolin-3-yl]-amino\}-acetic Acid Methyl Ester (266)

${ }^{1} \mathrm{H}$ NMR $\left(300 \mathrm{MHz}\right.$, methanol- $\left.d_{4}\right) \delta 8.92(\mathrm{~s}, 1 \mathrm{H}), 7.80(\mathrm{~s}, 1 \mathrm{H}), 7.75(\mathrm{~s}, 1 \mathrm{H}), 7.52(\mathrm{dd}, J=1.2$, $8.4 \mathrm{~Hz}, 2 \mathrm{H}), 7.44(\mathrm{~s}, 1 \mathrm{H}), 7.36(\mathrm{t}, J=7.5 \mathrm{~Hz}, 3 \mathrm{H}), 7.30-7.25(\mathrm{~m}, 2 \mathrm{H}), 6.85(\mathrm{~d}, J=8.7 \mathrm{~Hz}, 1 \mathrm{H})$, $4.75(\mathrm{~d}, J=16.8 \mathrm{~Hz}, 1 \mathrm{H}), 4.55(\mathrm{~d}, J=16.8 \mathrm{~Hz}, 1 \mathrm{H}), 4.50-4.40(\mathrm{~m}, 1 \mathrm{H}), 4.15(\mathrm{~d}, J=18.3 \mathrm{~Hz}$, $1 \mathrm{H}), 4.08(\mathrm{~d}, J=18.6 \mathrm{~Hz}, 1 \mathrm{H}), 3.95(\mathrm{~s}, 3 \mathrm{H}), 3.80(\mathrm{~s}, 3 \mathrm{H}), 3.70(\mathrm{~s}, 3 \mathrm{H}), 3.55-3.45(\mathrm{~m}, 2 \mathrm{H})$, 3.05-2.90 (m, 2H). MS m/z $535.4\left(\mathrm{M}+\mathrm{H}^{+}\right)$. General Procedure for the Synthesis of Compounds According to Scheme 7. General
Procedure for the Conversion of Nitrile (5) to Amide (28)

To a stirred solution of nitrile 5 (441 $\mathrm{mg}, 1 \mathrm{mmol})$ in alcohol $(8 \mathrm{~mL})$ was slowly added concentrated sulfuric acid $(0.05 \mathrm{~mL})$ at room temperature. The resulting solution was stirred at $40{ }^{\circ} \mathrm{C}$ for $2 \mathrm{~h}$. The mixture was poured into cold aqueous $20 \% \mathrm{KHCO}_{3}$ solution to neutralize the acid and precipitate the product. The product was filtered, washed with cold water, and dried under vacuum to give amides $28(40-45 \%)$.

3-(1-Methyl-1 $H$-imidazole-4-sulfonylamino)-1-(3-methyl-3 $H$-imidazol-4-ylmethyl)-1,2,3,4tetrahydroquinoline-6-carboxylic Acid tert-Butylamide (28)

${ }^{1} \mathrm{H}$ NMR $\left(300 \mathrm{MHz}\right.$, methanol- $\left.d_{4}\right) \delta 8.90(\mathrm{~s}, 1 \mathrm{H}), 7.80(\mathrm{~s}, 1 \mathrm{H}), 7.70(\mathrm{~s}, 1 \mathrm{H}), 7.51(\mathrm{dd}, J=2.1$, $8.7 \mathrm{~Hz}, 1 \mathrm{H}), 7.45(\mathrm{~s}, 1 \mathrm{H}), 7.40(\mathrm{~d}, J=2.1 \mathrm{~Hz}, 1 \mathrm{H}), 6.80(\mathrm{~d}, J=8.7 \mathrm{~Hz}, 1 \mathrm{H}), 4.80(\mathrm{~d}, J=16.8$ $\mathrm{Hz}, 1 \mathrm{H}), 4.60$ (d, $J=16.8 \mathrm{~Hz}, 1 \mathrm{H}), 3.92(\mathrm{~s}, 3 \mathrm{H}), 3.86-3.83$ (m, 1H), 3.80 (s, 1H), 3.52-3.45 (m, 1H), 3.42-3.35 (m, 1H), $3.0(\mathrm{dd}, J=4.8,16.5 \mathrm{~Hz}, 1 \mathrm{H}), 2.74(\mathrm{dd}, J=6.3,16.2 \mathrm{~Hz}, 1 \mathrm{H})$, $1.45(\mathrm{~s}, 9 \mathrm{H}) \mathrm{MS} m / z 486.4\left(\mathrm{M}+\mathrm{H}^{+}\right)$.

3-[(tert-Butylcarbamoyl-methyl)-(1-methyl-1 H-imidazol-4-sulfonyl)-amino]-1-(3-methyl-3Himidazol-4-ylmethyl)-1,2,3,4-tetrahydro-quinolin-6-carboxylic Acid tert-Butylamide (285)

The compound was prepared using the general $\mathrm{N}$-alkylation procedure (5 to 2 , Scheme 1 ). ${ }^{1} \mathrm{H}$ NMR $\left(300 \mathrm{MHz}\right.$, methanol- $\left.d_{4}\right) \delta 8.92(\mathrm{~s}, 1 \mathrm{H}), 7.85(\mathrm{~s}, 1 \mathrm{H}), 7.80(\mathrm{~s}, 1 \mathrm{H}), 7.52(\mathrm{dd}, J=2.1,8.7$ $\mathrm{Hz}, 1 \mathrm{H}), 7.43(\mathrm{~s}, 1 \mathrm{H}), 7.40(\mathrm{~d}, J=2.1 \mathrm{~Hz}, 1 \mathrm{H}), 6.79(\mathrm{~d}, J=8.7 \mathrm{~Hz}, 1 \mathrm{H}), 4.75(\mathrm{~d}, J=16.8 \mathrm{~Hz}$, $1 \mathrm{H}), 4.63(\mathrm{~d}, J=16.8 \mathrm{~Hz}, 1 \mathrm{H}), 4.33-4.25(\mathrm{~m}, 1 \mathrm{H}), 3.92(\mathrm{~s}, 3 \mathrm{H}), 3.87(\mathrm{~s}, 2 \mathrm{H}), 3.81(\mathrm{~s}, 3 \mathrm{H})$, $3.57-3.51(\mathrm{~m}, 1 \mathrm{H}), 3.44-3.40(\mathrm{~m}, 1 \mathrm{H}), 3.03(\mathrm{dd}, J=4.5,15.9 \mathrm{~Hz}, 1 \mathrm{H}), 2.80(\mathrm{dd}, J=4.5,15.9$ $\mathrm{Hz}, 1 \mathrm{H}), 1.43$ (s, 9H), 1.35 (s, 9H). MS m/z $599.5\left(\mathrm{M}+\mathrm{H}^{+}\right)$.

\section{General Procedure for the Conversion of Nitrile (5) to Acid (27)}

A solution of $5(441 \mathrm{mg}, 1.0 \mathrm{~m} \mathrm{~mol})$ in concentrated $\mathrm{HCl}(20 \mathrm{~mL})$ was stirred at $80^{\circ} \mathrm{C}$ for 2 $\mathrm{h}$. The reaction mixture was cooled to room temperature and then evaporated to dryness under reduced pressure. The residue was dissolved in saturated $\mathrm{LiOH}$ solution $(\mathrm{pH} 9)$ and then 
evaporated to dryness under reduced pressure. The residue was dissolved in $10 \%$ aqueous $\mathrm{HCl}$ solution ( $\mathrm{pH}$ 2), evaporated, and dried under vacuum to give the corresponding acid 27, which was used in the next step without further purification. ${ }^{1} \mathrm{H} \mathrm{NMR}\left(300 \mathrm{MHz}\right.$, methanol- $\left.d_{4}\right) \delta$ $8.90(\mathrm{~s}, 1 \mathrm{H}), 7.80(\mathrm{~s}, 1 \mathrm{H}), 7.70(\mathrm{~s}, 1 \mathrm{H}), 7.62(\mathrm{dd}, J=2.1,8.7 \mathrm{~Hz}, 1 \mathrm{H}), 7.52(\mathrm{~d}, J=2.1 \mathrm{~Hz}, 1 \mathrm{H})$, $7.42(\mathrm{~s}, 1 \mathrm{H}), 6.80(\mathrm{~d}, J=8.7 \mathrm{~Hz}, 1 \mathrm{H}), 4.80(\mathrm{~d}, J=16.8 \mathrm{~Hz}, 1 \mathrm{H}), 4.62(\mathrm{~d}, J=16.8 \mathrm{~Hz}, 1 \mathrm{H})$, $3.92(\mathrm{~s}, 3 \mathrm{H}), 3.90-3.82(\mathrm{~m}, 1 \mathrm{H}), 3.80(\mathrm{~s}, 3 \mathrm{H}), 3.55-3.45(\mathrm{~m}, 1 \mathrm{H}), 3.43-3.38(\mathrm{~m}, 1 \mathrm{H}), 3.03$ (dd, $J=4.8,16.5 \mathrm{~Hz}, 1 \mathrm{H}), 2.76(\mathrm{dd}, J=6.3,16.2 \mathrm{~Hz}, 1 \mathrm{H}) . \mathrm{MS} m / z 486.4\left(\mathrm{M}+\mathrm{H}^{+}\right)$.

To a mixture of the acid 27 (430 $\mathrm{mg}, 1.0 \mathrm{~m} \mathrm{~mol})$ above, benzyl amine (107 $\mathrm{mg}, 1 \mathrm{mmol}$ ), and $N, N$-dimethylamino pyridine ( $18 \mathrm{mg}, 0.15 \mathrm{mmol}$ ) in DMF was added EDC (287 mg, $1.5 \mathrm{mmol})$, and the mixture was stirred at room temperature for $20 \mathrm{~h}$. The mixture was concentrated under reduced pressure, and aqueous $\mathrm{NaHCO}_{3}$ solution $(20 \mathrm{~mL})$ was added. The mixture was extracted with $10 \% \mathrm{MeOH}$ in $\mathrm{CHCl}_{3}(3 \times 30 \mathrm{~mL})$, and the organic layer was dried $\left(\mathrm{Na}_{2} \mathrm{SO}_{4}\right)$ and evaporated under reduced pressure. The crude product was purified by flash chromatography to afford $\mathbf{2 8}(220 \mathrm{mg}, 38 \%)$.

\section{3-(1-Methyl-1H-imidazole-4-sulfonylamino)-1-(3-methyl-3H-imidazol-4-ylmethyl)-1,2,3,4- tetrahydroquinoline-6-carboxylic Acid Benzylamide (28)}

${ }^{1} \mathrm{H}$ NMR $(300 \mathrm{MHz}$, methanol-d $) \delta 8.90(\mathrm{~s}, 1 \mathrm{H}), 7.76(\mathrm{~s}, 1 \mathrm{H}), 7.70(\mathrm{~s}, 1 \mathrm{H}), 7.62(\mathrm{dd}, J=2.1$, $8.7 \mathrm{~Hz}, 1 \mathrm{H}), 7.50(\mathrm{~d}, J=2.1 \mathrm{~Hz}, 1 \mathrm{H}), 7.46(\mathrm{~s}, 1 \mathrm{H}), 7.36-7.30(\mathrm{~m}, 5 \mathrm{H}), 6.82(\mathrm{~d}, J=8.7 \mathrm{~Hz}$, $1 \mathrm{H}), 4.75(\mathrm{~d}, J=16.8 \mathrm{~Hz}, 1 \mathrm{H}), 4.62(\mathrm{~d}, J=16.8 \mathrm{~Hz}, 1 \mathrm{H}), 4.55(\mathrm{~s}, 2 \mathrm{H}), 3.92(\mathrm{~s}, 3 \mathrm{H}), 3.90-3.82$ $(\mathrm{m}, 1 \mathrm{H}), 3.80(\mathrm{~s}, 3 \mathrm{H}), 3.53-3.48(\mathrm{~m}, 1 \mathrm{H}), 3.43-3.39(\mathrm{~m}, 1 \mathrm{H}), 3.03(\mathrm{dd}, J=4.8,16.5 \mathrm{~Hz}, 1 \mathrm{H})$, $2.75(\mathrm{dd}, J=6.3,16.2 \mathrm{~Hz}, 1 \mathrm{H})$.

\section{3-[(tert-Butylcarbamoyl-methyl)-(1-methyl-1 H-imidazol-4-sulfonyl)-amino]-1-(3-methyl-3 H- imidazol-4-ylmethyl)-1,2,3,4-tetrahydro-quinolin-6-carboxylic Acid Benzylamide (291)}

Compound 291 was prepared as described from the general procedure for $N$-alkylation. ${ }^{1} \mathrm{H}$ NMR $\left(300 \mathrm{MHz}\right.$, methanol- $\left.d_{4}\right) \delta 8.92(\mathrm{~s}, 1 \mathrm{H}), 7.80(\mathrm{~s}, 1 \mathrm{H}), 7.78(\mathrm{~s}, 1 \mathrm{H}), 7.63(\mathrm{dd}, J=2.1,8.7$ $\mathrm{Hz}, 1 \mathrm{H}), 7.53(\mathrm{~d}, J=2.1 \mathrm{~Hz}, 1 \mathrm{H}), 7.42(\mathrm{~s}, 1 \mathrm{H}), 7.36-7.30(\mathrm{~m}, 5 \mathrm{H}), 6.82(\mathrm{~d}, J=8.7 \mathrm{~Hz}, 1 \mathrm{H})$, $4.77(\mathrm{~d}, J=16.8 \mathrm{~Hz}, 1 \mathrm{H}), 4.65(\mathrm{~d}, J=17.4 \mathrm{~Hz}, 1 \mathrm{H}), 4.55(\mathrm{~s}, 2 \mathrm{H}), 4.33-4.22(\mathrm{~m}, 1 \mathrm{H}), 3.91(\mathrm{~s}$, $3 \mathrm{H}), 3.85$ (s, 2H), $3.80(\mathrm{~s}, 3 \mathrm{H}), 3.56-3.50(\mathrm{~m}, 1 \mathrm{H}), 3.46-3.39(\mathrm{~m}, 1 \mathrm{H}), 3.03(\mathrm{dd}, J=5.1,15.3$ $\mathrm{Hz}, 1 \mathrm{H}), 2.86(\mathrm{dd}, J=5.1,15.3 \mathrm{~Hz}, 1 \mathrm{H}), 1.4(\mathrm{~s}, 9 \mathrm{H}) . \mathrm{MS} m / z 633.8\left(\mathrm{M}+\mathrm{H}^{+}\right)$.

\section{3-[(tert-Butylcarbamoyl-methyl)-(1-methyl-1 H-imidazol-4-sulfonyl)-amino]-1-(3-methyl-3 H- imidazol-4-ylmethyl)-1,2,3,4-tetrahydro-quinolin-6-carboxylic Acid (289)}

Compound 289 was prepared as described from general procedure for $N$-alkylation. ${ }^{1} \mathrm{H}$ NMR $\left(300 \mathrm{MHz}\right.$, methanol- $\left.d_{4}\right) \delta 8.92(\mathrm{~s}, 1 \mathrm{H}), 7.82(\mathrm{~s}, 1 \mathrm{H}), 7.79(\mathrm{~s}, 1 \mathrm{H}), 7.62(\mathrm{dd}, J=2.1,8.7 \mathrm{~Hz}$, $1 \mathrm{H}), 7.52(\mathrm{~d}, J=2.1 \mathrm{~Hz}, 1 \mathrm{H}), 7.42(\mathrm{~s}, 1 \mathrm{H}), 6.80(\mathrm{~d}, J=8.7 \mathrm{~Hz}, 1 \mathrm{H}), 4.75(\mathrm{~d}, J=16.8 \mathrm{~Hz}, 1 \mathrm{H})$, $4.65(\mathrm{~d}, J=16.8 \mathrm{~Hz}, 1 \mathrm{H}), 4.35-4.25(\mathrm{~m}, 1 \mathrm{H}), 3.92(\mathrm{~s}, 3 \mathrm{H}), 3.85(\mathrm{~s}, 2 \mathrm{H}), 3.80(\mathrm{~s}, 3 \mathrm{H}), 3.60-$ $3.51(\mathrm{~m}, 1 \mathrm{H}), 3.50-3.40(\mathrm{~m}, 1 \mathrm{H}), 3.03(\mathrm{dd}, J=4.8,16.5 \mathrm{~Hz}, 1 \mathrm{H}), 2.80(\mathrm{dd}, J=6.3,16.2 \mathrm{~Hz}$, $1 \mathrm{H}), 1.40(\mathrm{~s}, 9 \mathrm{H}) . \mathrm{MS} \mathrm{m} / \mathrm{z} 543.5\left(\mathrm{M}+\mathrm{H}^{+}\right)$.

\section{Synthesis of 34 According to Scheme 8. Preparation of 1-[4-( $N$-triphenylmethyl)imidazolyl] ethanol}

To an ice-bath cooled solution of $3.35 \mathrm{~mL}(10.0 \mathrm{mmol})$ of $3 \mathrm{M}$ methyl magnesium bromide in $40 \mathrm{~mL}$ dry ether was added a solution of 4-( $N$-triphenylmethyl)-imidazole carboxaldehyde $(1.69 \mathrm{~g}, 10 \mathrm{mmol})$ in THF. After $1.5 \mathrm{~h}$ at ambient temperature, a concentrated solution of 0.67 $\mathrm{g}(25 \mathrm{mmol}) \mathrm{NH}_{4} \mathrm{Cl}$ in water was added to the reaction mixture. The mixture was stirred for 1 $\mathrm{h}$ and filtered, and the solids were washed with THF. The combined filtrate and washes were washed with water and brine, dried over $\mathrm{NaSO}_{4}$, and concentrated in vacuo to afford the 
required compound. ${ }^{17}{ }^{1} \mathrm{H}$ NMR $\left(300 \mathrm{MHz} \mathrm{CDCl}_{3}\right) 7.42(\mathrm{~s}, 1 \mathrm{H}), 7.30-7.39(\mathrm{~m}, 9 \mathrm{H}), 7.10-7.21$ (m, 6H), 6.85 (s, 1H), $4.85(\mathrm{q}, 1 \mathrm{H}), 1.5(\mathrm{~d}, 3 \mathrm{H}) . \mathrm{MS} \mathrm{m} / z 355.2\left(\mathrm{M}+\mathrm{H}^{+}\right)$.

A mixture of $\mathbf{3 3}$ (47 mg, $0.1 \mathrm{mmol})$ and 1-(4-( $N$-triphenylmethyl)imidazolyl)ethanol (17 mg, $0.05 \mathrm{mmol}$ ) in $5 \mathrm{~mL}$ of dry acetonitrile was added methanesulfonyl chloride ( $34 \mathrm{mg}, 0.3 \mathrm{mmol}$ ), and the mixture was heated at $60{ }^{\circ} \mathrm{C}$ overnight under nitrogen. Aqueous $\mathrm{NaHCO}_{3}$ and ethyl acetate were added. The organic layer was washed with water and brine and dried over $\mathrm{MgSO}_{4}$. The crude product was purified by HPLC to give 34 as a mixture of isomers. ${ }^{18}{ }^{1} \mathrm{H}$ NMR $\left(300 \mathrm{MHz}\right.$, methanol- $\left.d_{4}\right) \delta 8.95(\mathrm{~s}, 1 \mathrm{H}), 8.94(\mathrm{~s}, 1 \mathrm{H}), 8.72(\mathrm{~d}, J=7.5 \mathrm{~Hz}, 1 \mathrm{H}), 8.68(\mathrm{~d}$, $J=7.5 \mathrm{~Hz}, 1 \mathrm{H}), 7.90-8.10(\mathrm{~m}, 4 \mathrm{H}), 7.59-7.70(\mathrm{~m}, 4 \mathrm{H}), 7.31-7.48(\mathrm{~m}, 4 \mathrm{H}), 6.95(\mathrm{~d}, J=8.7$ $\mathrm{Hz}, 1 \mathrm{H}), 6.91(\mathrm{~d}, J=8.7 \mathrm{~Hz}, 1 \mathrm{H}), 5.50(\mathrm{q}, 1 \mathrm{H}), 5.40(\mathrm{q}, 1 \mathrm{H}), 4.38-4.51(\mathrm{~m}, 2 \mathrm{H}), 4.08-4.16$ $(\mathrm{m}, 4 \mathrm{H}), 3.70(\mathrm{~s}, 6 \mathrm{H}), 3.32-3.45(\mathrm{~m}, 4 \mathrm{H}), 3.01-3.22(\mathrm{~m}, 6 \mathrm{H}), 2.65-2.90(\mathrm{~m}, 6 \mathrm{H}), 1.68-1.88$ $(\mathrm{m}, 6 \mathrm{H}), 1.60(\mathrm{~d}, 6 \mathrm{H}), 1.05-1.17(\mathrm{~m}, 4 \mathrm{H}) . \mathrm{MS} m / z 564.6\left(\mathrm{M}+\mathrm{H}^{+}\right)$.

\section{Resolution of Enantiomers (163 and 164)}

Enantiomerically pure amines derived from racemic 6-cyano-1,2,3,4-tetrahydro-quinolin-3ylamine hydrochloride were obtained by coupling with $S$-mandelic acid to form diastereomeric amides as described previously. ${ }^{5,10}$ After resolution, the amines were converted to $\mathbf{1 6 3}$ and 164, as shown in Scheme 1.

\section{Modeling Studies}

We published a detailed procedure for building a homology model of Pf-PFT.7 Briefly, the model was generated with MODELLER19 by using the crystal structure of rat-PFT complexed with the nonsubstrate tetrapeptide inhibitor CVFM and farnesyl-pyrophosphate as the template structure (PDB entry 1JCR). The sequences of the two subunits ( $\alpha$ and $\beta$ ) of Pf-PFT were obtained from the PlasmoDB ${ }^{20}(\alpha$ : PFL2050w and $\beta$ : chr11.glm_528) and aligned with the template. Only regions with reasonable reliability in the alignment were included. The model of Pf-PFT comprises the following sequence segments (the residue numbers of the corresponding segments of the rat-PFT subunits are given in parentheses): $\alpha$ : 72-164 (87-179), 300-411 (184-283); $\beta$ : 421-677 (71-315), 806-896 (330-417). Note that the Pf-PFT sequences $(\alpha: 497 ; \beta: 967)$ are much longer than the rat ones $(\alpha: 377 ; \beta: 437)$

A small molecule crystal structure of 7-nitro-1,2,3,4-THQ, ${ }^{21}$ with $R=0.034$ at $0.77 \AA$ resolution, shows that the tetrahydropyridine ring adopts an envelope conformation, with atom C3 deviating by $0.66 \AA$ from the mean plane through the other five atoms. Upon substitution with an equatorial amino group, the deviation of the methyl from the plane is $0.40 \AA$, implying that when the two enantiomers are superimposed the amines are only $0.40 \AA$ apart. Both conformers of the molecule were built with Insight (Accelrys) starting from the 7-nitro-1,2,3,4THQ crystal structure, and the energies were minimized with the CFF force-field; a 4re electrostatics model was used. The energies were $54.28 \mathrm{kcal} / \mathrm{mol}$ (equatorial) and $56.96 \mathrm{kcal} /$ mol (axial).

All molecular docking calculations of the THQ compounds were carried out using the FLO/ QXP v.6.02 package ${ }^{24}$ with as start model 162, as observed in the rat-PFT crystal structure transferred by superposition into the comparative model of Pf-PFT. THQ compounds were built with the FLO BUILDER module, specifically taking care to assign the mixed sp2/sp3 N atom type of the sulfonamide and allowing for mild pyramidalization. Monte Carlo searches were carried out for the THQ substituents, using 50 cycles per rotatable bond, while keeping the THQ scaffold fixed. Subsequently, the best solutions were minimized, allowing also for spatial freedom to the scaffold. Because the force-field has not been parametrized for transition metal complexes, the inhibitor $\mathrm{N}$ atom that coordinates the $\mathrm{Zn}^{2+}$ atom was spatially constrained as well as all other atoms of the $\mathrm{Zn}^{2+}$-binding imidazole ring. 


\section{Plasmodium Strains}

The P. falciparum strains used in this study were 3D7 (Netherlands [airport-associated malaria], chloroquine sensitive) and K1 (Thailand, chloroquine resistant, pyrimethamine resistant). Strain 3D7 was provided by Dr. Pradipsinh Rathod from the University of Washington. P. falciparum strain K1 and P. berghei isolate NK65 (used for rodent malaria experiments) were obtained from the MR4 unit of the American Type Culture Collection (ATCC, Manassas, VA).

\section{P. falciparum Culture}

Strains of $P$. falciparum were cultured in vitro using experimental techniques described by Trager and Jensen. ${ }^{22}$ Cultures were maintained in RPMI-1640 (Sigma, St. Louis, MI) with 2 $\mathrm{mM}$ L-glutamine, $25 \mathrm{mM}$ HEPES, $33 \mathrm{mM} \mathrm{NaHCO} 3,20 \mu \mathrm{g} / \mathrm{mL}$ gentamicin sulfate, and $20 \%$ $(\mathrm{v} / \mathrm{v})$ heat-inactivated human plasma type A+ (RP-20P). Type A+ erythrocytes were obtained from lab donors, washed three times with RPMI 1640, resuspended in 50\% RPMI-1640, and stored at $4{ }^{\circ} \mathrm{C}$. Parasites were grown in $10 \mathrm{~mL}$ of a $2 \%$ hematocrit/RP-20P $(\mathrm{v} / \mathrm{v})$ in $50 \mathrm{~mL}$ flasks under a $5 \% \mathrm{CO}_{2}, 5 \% \mathrm{O}_{2}$, and $90 \% \mathrm{~N}_{2}$ atmosphere.

\section{P. falciparum $\mathrm{ED}_{50}$ Determination}

A total of $10 \mu L$ of PFTI in solution was added to each well of a 96-well plate followed by the addition of $190 \mu L$ of an asynchronous $P$. falciparum culture at parasitemia and hematocrit of $0.5 \%$. PFTI solutions were prepared by diluting $20 \mathrm{mM}$ THQ PFTI in dimethylsulfoxide by 200-fold with RP-20P for the highest concentration (100 $\mu$ M stock gives final assay concentration of $5 \mu \mathrm{M}$ ), then performing further serial dilutions in RP-20P. Plates were flushed with $5 \% \mathrm{CO}_{2}, 5 \% \mathrm{O}_{2}$, and $90 \% \mathrm{~N}_{2}$ then incubated at $37^{\circ} \mathrm{C}$ for 48 h. $8\left[{ }^{3} \mathrm{H}\right]$-Hypoxanthine $(0.3$ $\mu \mathrm{Ci}, 20 \mathrm{Ci} / \mathrm{mmol}$, American Radiolabeled Chemicals) in $30 \mu \mathrm{L}$ of RP-20P was added to cultures and incubated for an additional $24 \mathrm{~h}$. Cells were harvested onto glass fiber filters by a cell harvester (Inotech Biosystems International, Inc Rockville, MD), and the radioactivity incorporated into the parasites was counted on a Chameleon 425-104 multilabel plate counter (Hidex Oy Turku, Finland). The background level detected with uninfected erythrocytes was subtracted from the data. The ${ }^{3} \mathrm{H}$-incorporation into infected erythrocytes with $1 \mu \mathrm{L}$ of DMSO vehicle alone represents $100 \%$ malaria growth. $\mathrm{ED}_{50}$ values, the effective dose that reduces growth by $50 \%$, were determined by linear regression analysis of the plots of ${ }^{3} \mathrm{H}$-hypoxanthine incorporation versus concentration of compound. Each compound was tested in duplicate, and the mean value is shown; individual measurements differed by less than 3-fold.

\section{Pf-PFT IC 50 Determination}

The PFT assay used to determine the $\mathrm{IC}_{50} \mathrm{~s}$ (inhibitor concentration that causes $50 \%$ enzyme inhibition) of the compounds is based on a PFT $\left[{ }^{3} \mathrm{H}\right]$ scintillation proximity assay (SPA; TRKQ7010 Amersham Biosciences Corp Piscataway, NJ). ${ }^{3}$ Assays were carried out in assay buffer (pH 7.5, $50 \mathrm{mM}$ HEPES, $30 \mathrm{mM} \mathrm{MgCl}_{2}, 20 \mathrm{mM} \mathrm{KCl}, 5 \mathrm{mM}$ DTT, $0.01 \%$ Triton X-100), $1 \mu \mathrm{M}$ human lamin-B carboxy-terminus sequence peptide (biotin-YRASNRSCAIM), and 1 $\mu \mathrm{Ci}{ }^{3} \mathrm{H}$-farnesylpyrophos-phate (Amersham specific activity 15 to $20 \mathrm{Ci} / \mathrm{mM}$ ) in a total volume of $50 \mu \mathrm{L}$, which included $1 \mu \mathrm{L}$ of Pf-PFT inhibitor solution in DMSO and $5 \mu \mathrm{L}$ of partially purified Pf-PFT. Assays in the absence of Pf-PFT inhibitor and Pf-PFT were included as positive and negative controls, respectively. Reaction mixtures were incubated at $37^{\circ} \mathrm{C}$ for 60 min and terminated by the addition of $70 \mu \mathrm{L}$ of assay STOP solution and $5 \mu \mathrm{L}$ of SPA beads. The assay mixture was incubated at room temperature for $30 \mathrm{~min}$. The assay was counted on a plate Chameleon 425-104 multilabel counter (Hidex Oy Turku, Finland). IC $_{50}$ values were calculated using linear regression analysis of the plots of the amount of radio-prenylation versus the concentration of compound. 


\section{Mammalian-PFT IC 50 Determination}

The PFT assay used to determine the $\mathrm{IC}_{50} \mathrm{~s}$ of the compounds is based on a farnesyl transferase $\left[{ }^{3} \mathrm{H}\right]$ SPA enzyme assay (TRKQ7010 Amersham Biosciences Corp Piscataway, NJ). Rat PFT was prepared as described. ${ }^{23}$ Assays were carried out in assay buffer $\mathrm{pH} 7.5$ (50 mM HEPES, $30 \mathrm{mM} \mathrm{MgCl} 2,20 \mathrm{mM} \mathrm{KCl}, 5 \mathrm{mM}$ DTT, $0.01 \%$ Triton X-100) and $1 \mu \mathrm{M}$ human lamin-B carboxy-terminus sequence peptide (biotin-YRASNRSCAIM) in a total volume of $50 \mu \mathrm{L}$, which included $1 \mu \mathrm{L}$ of PFT inhibitor solution in DMSO and $5 \mu \mathrm{L}$ of partially purified ratPFT. Assays in the absence of PFT inhibitor and rat-PFT were included as positive and negative controls, respectively. Reaction mixtures were incubated at $37^{\circ} \mathrm{C}$ for $15 \mathrm{~min}$ and terminated by the addition of $70 \mu \mathrm{L}$ of assay STOP solution and $5 \mu \mathrm{L}$ of SPA beads. The assay is incubated at room temperature for $30 \mathrm{~min}$. The assay was counted on a plate Chameleon 425-104 multilabel counter (Hidex Oy Turku, Finland). $\mathrm{IC}_{50}$ values were calculated using linear regression analysis of the plots of ${ }^{3} \mathrm{H}-\mathrm{FPP}$ prenylation versus concentration of compounds.

\section{References}

1. Chakrabarti D, Azam T, DelVecchio C, Qiu L, Park Y, Allen CM. Protein prenyl transferase activities of Plasmodium falciparum. Mol. Biochem. Parasitol 1998;94:175-184. [PubMed: 9747968]

2. Chakrabarti D, Da Silva T, Barger J, Paquette S, Patel H, Patterson S, Allen CM. Protein farnesyltransferase and protein prenylation in Plasmodium falciparum. J. Biol. Chem 2002;277:42066-42073. [PubMed: 12194969]

3. Nallan L, Bauer KD, Bendale P, Rivas K, Yokoyama K, Horney CP, Pendyala PR, Floyd D, Lombardo LJ, Williams DK, Hamilton A, Sebti S, Windsor WT, Weber PC, Buckner FS, Chakrabarti D, Gelb $\mathrm{MH}$, Van Voorhis WC. Protein farnesyltransferase inhibitors exhibit potent antimalarial activity. J. Med. Chem 2005;48:3704-3713. [PubMed: 15916422]

4. Bell IM. Inhibitors of farnesyltransferase: A rational approach to cancer chemotherapy? J. Med. Chem 2004;47:1869-1878. [PubMed: 15055985]

5. Lombardo LJ, Camuso A, Clark J, Fager K, Gullo-Brown J, Hunt JT, Inigo I, Kan D, Koplowitz B, Lee F, McGlinchey K, Qian L, Ricca C, Rovnyak G, Traeger S, Tokarski J, Williams DK, Wu LI, Zhao Y, Manne V, Bhide RS. Design, synthesis, and structure-activity relationships of tetrahyroquinoline-based farnesyltransferase inhibitors. Bioorg. Med. Chem. Lett 2005;15:18951899. [PubMed: 15780629]

6. Hunt JT, Ding CZ, Batorsky R, Bednarz M, Bhide R, Cho Y, Chong S, Chao S, Gullo-Brown J, Guo P, Kim SH, Lee FY, Leftheris K, Miller A, Mitt T, Patel M, Penhallow BA, Ricca C, Rose WC, Schmidt R, Slusarchyk WA, Vite G, Manne V. Discovery of $(R)$-7-cyano-2,3,4,5-tetrahydro-1-(1H-imidazol-4ylmethyl)-3-phenylmethyl)-4-(2-thienylsulfonyl)-1H-1,4-benzodiazepine (BMS-214662), a farnesyltransferase inhibitor with potent preclinical antitumor activity. J. Med. Chem 2000;43:35873595. [PubMed: 11020273]

7. Eastman RT, White J, Hucke O, Bauer K, Yokoyama K, Nallan L, Chakrabarti D, Verlinde CL, Gelb MH, Rathod PK, Van Voorhis WC. Resistance to a protein farnesyltransferase inhibitor in Plasmodium falciparum. J. Biol. Chem 2005;280:13554-13559. [PubMed: 15661734]

8. Eastman RT, White J, Hucke O, Yokoyama K, Verlinde CL, Hast MA, Beese LS, Gelb MH, Rathod PK, Van Voorhis WC. Resistance mutations at the lipid substrate binding site of Plasmodium falciparum protein farnesyltransferase. Mol. Biochem. Parasitol 2007;152(1):66-71. [PubMed: 17208314]

9. Van Voorhis WC, Rivas K, Bendale P, Nallan L, Hornéy C, Barrett LK, Ankala S, Hucke O, Verlinde CLMJ, Chakrabarti D, Strickland C, Yokoyama K, Buckner FS, Hamilton A, Williams DK, Lombardo LJ, Floyd D, Gelb MH. Tetrahydroquinoline Plasmodium falciparum protein farnesyltransferase inhibitors: Efficacy, pharmacokinetics, and metabolism. Antimicrob. Agents Chemother. 2007 in press.

10. Bhide R. Inhibitors of farnesyl protein transferase. 1999 WO 99/01434.

11. Reid TS, Beese LS. Crystal structures of the anticancer clinical candidates R115777 (Tipifarnib) and BMS-214662 complexed with protein farnesyltransferase suggest a mechanism of FTI selectivity. Biochemistry 2004;43:6877-6884. [PubMed: 15170324] 
12. Nicholas JB, Vance REM, Burke BJ, Hopfinger AJ. A molecular mechanics valence force field for sulfonamides derived by ab initio methods. J. Chem. Phys 1991;95:9803-9811.

13. Liang GY, Bays JP, Bowen JP. Ab initio calculations and molecular mechanics (MM3) force field development for sulfonamide and its alkyl derivatives. Theochem 1997;40(1/2):165-179.

14. Hillal SH, Karickhoff SW, Carreira LA. A rigorous test for SPARC's chemical reactivity models: Estimation of more than 4300 ionization $\mathrm{p} K(\mathrm{a}) \mathrm{s}$. Quant. Struct.-Act. Relat 1995;14:348.

15. Hillal SH, El-Shabrawy Y, Carreira LA, Karickhoff WW, Toubar SS, Rizk M. Estimation of the ionization $\mathrm{p} K_{\mathrm{a}}$ of pharmaceutical substances using the computer program sparc. Talanta 1996;43:607-619. [PubMed: 18966526]

16. Chen BC, Skoumbourdis AP, Sundenen JE, Rovnyak GC, Traeger SC. Efficient molar-scale synthesis of 1-methyl-5-acylimidazole triflic acid salts. Org. Process Res. Dev 2000;4:513-614.

17. Kelley JL, Miller CA, McLean EW. Attempted inhibition of histidine decarboxylase with $\beta$-alkyl analogues of histidine. J. Med. Chem 1977;20:721-723. [PubMed: 853508]

18. Venishi J, Hamada M, Aburatani S, Matsui K, Yonemitsu O, Tsukube H. Synthesis of chiral nonracemic 1-(2-pyridinyl)ethylamines: Stereospecific introduction of amino function onto the 2pyridinylmethyl carbon center. J. Org. Chem 2004;69:6781-6789. [PubMed: 15387603]

19. Sali A, Blundell TL. Comparative protein modelling by satisfaction of spatial restraints. J. Mol. Biol 1993;234:779-815. [PubMed: 8254673]

20. Bahl A, Brunk B, Crabtree J, Fraunholz MJ, Gajria B, Grant GR, Ginsburg H, Gupta D, Kissinger JC, Labo P, Li L, Mailman MD, Milgram AJ, Pearson DS, Roos DS, Schug J, Stoeckert CJ Jr, Whetzel P. PlasmoDB: The Plasmodium genome resource. A database integrating experimental and computational data. Nucleic Acids Res 2003;31:212-215. [PubMed: 12519984]

21. Gu JM, Hu XR, Xu WM. 7-Nitro-1,2,3,4-tetrahydroquinoline. Acta Crystallogr 2006;E62:o62-o63.

22. Trager W, Jensen JB. Human malaria parasites in continuous culture. Science 1976;193:673-675. [PubMed: 781840]

23. Yokoyama K, Zimmerman K, Scholten J, Gelb MH. Differential prenyl pyrophosphate binding to mammalian protein geranylgeranyltransferase-I and protein farnesyltransferase and its consequence on the specificity of protein prenylation. J. Biol. Chem 1997;272:3944-3952. [PubMed: 9020098]

24. McMartin C, Bohacek RS. QXP: Powerful, rapid computer algorithms for structure-based drug design. J. Comput.-Aided Mol. Des 1997;11:333-344. [PubMed: 9334900] 

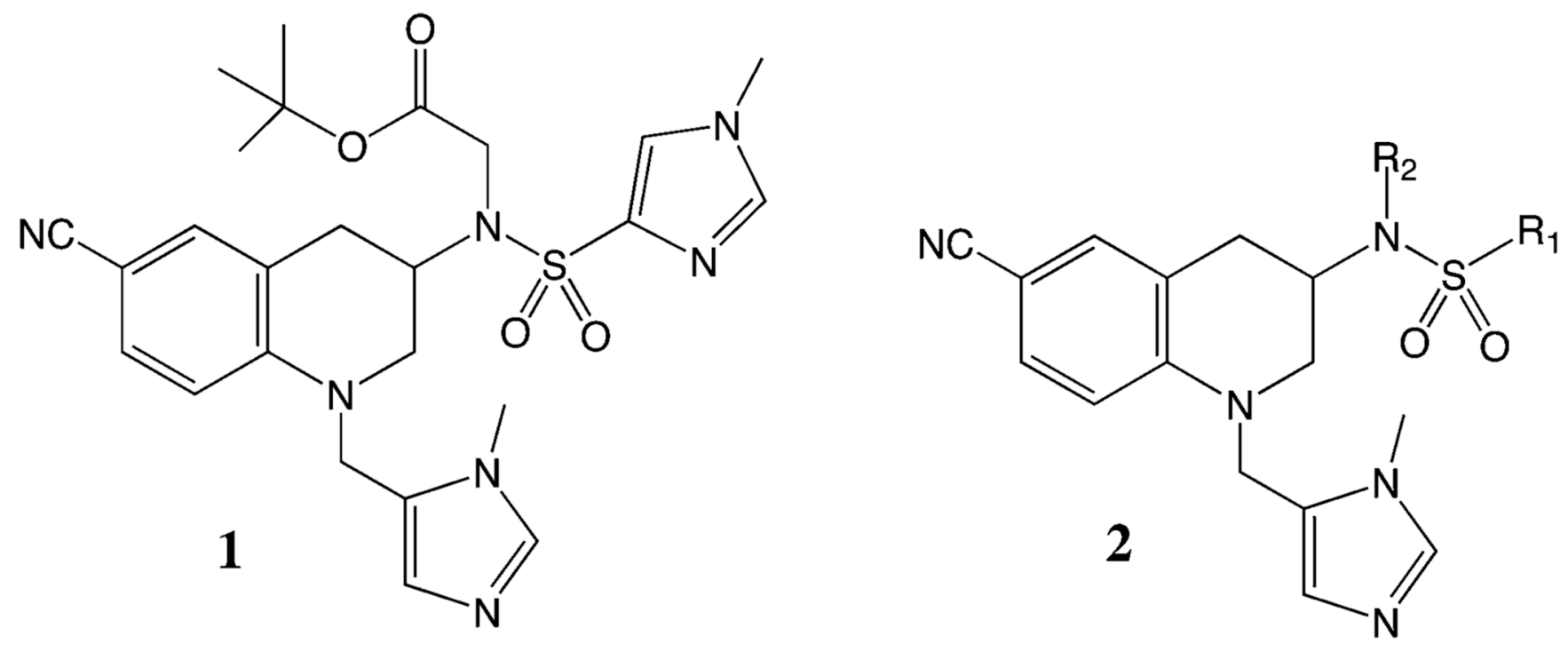

Figure 1.

Tetrahydroquinoline-based protein farnesyltransferase inhibitors. See main text for discussion. 


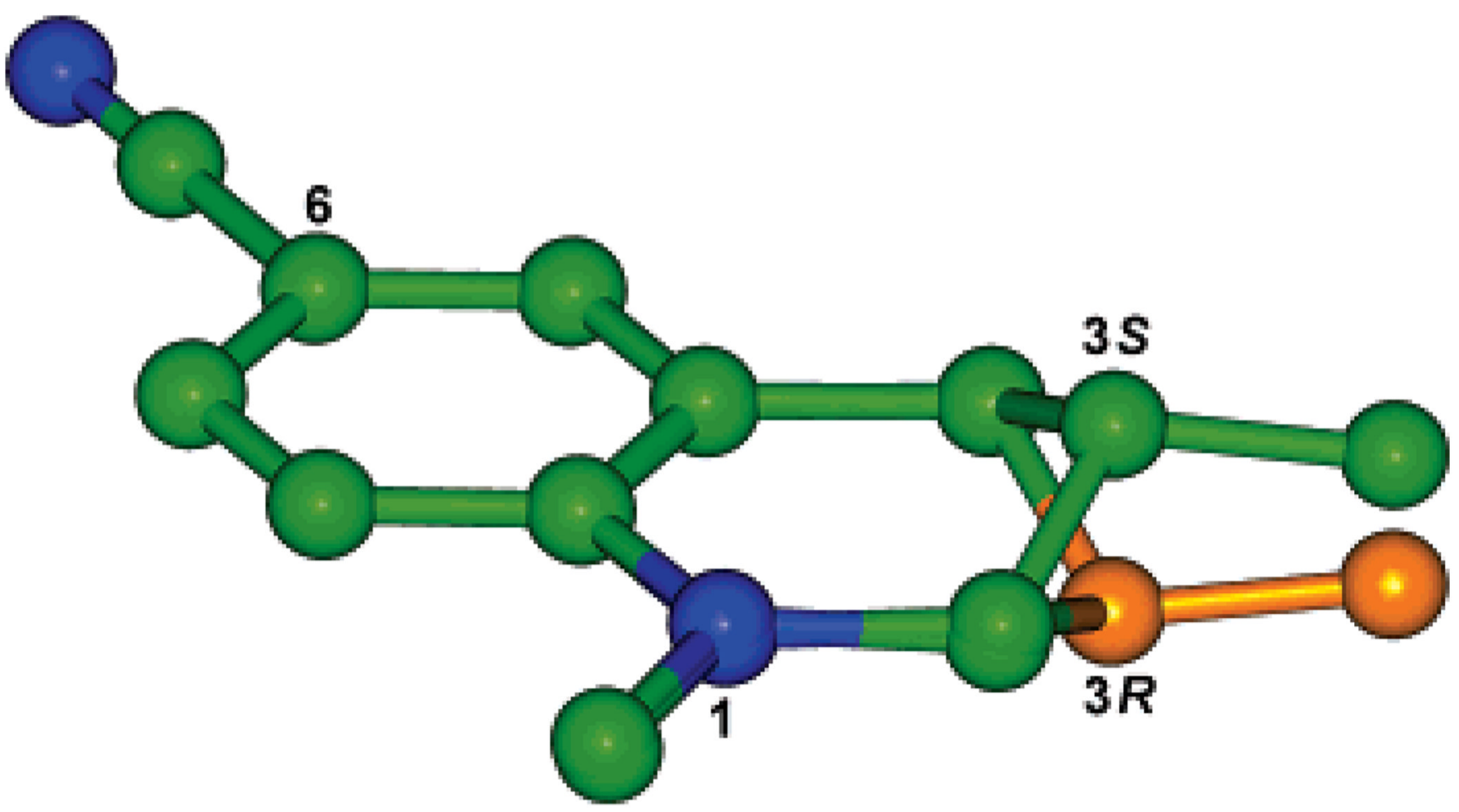

Figure 2.

Near coincidence of the sulfonamide nitrogen positions of the C3 enantiomers of THQ inhibitors like 162. The figure shows the superposition of the two possible envelope conformations of the THQ ring, resulting in the positions of the exocyclic sulfonamide nitrogens being only $0.8 \AA$ apart (only the equatorial conformations are shown; they are 2.7 $\mathrm{kcal} / \mathrm{mol}$ more stable than the axial conformations). The $R / S$ designation is specifically for the sulfonamide substitution. All figures of 3-dimensional molecular scenes were made with PyMOL (http://www.pymol.org). 

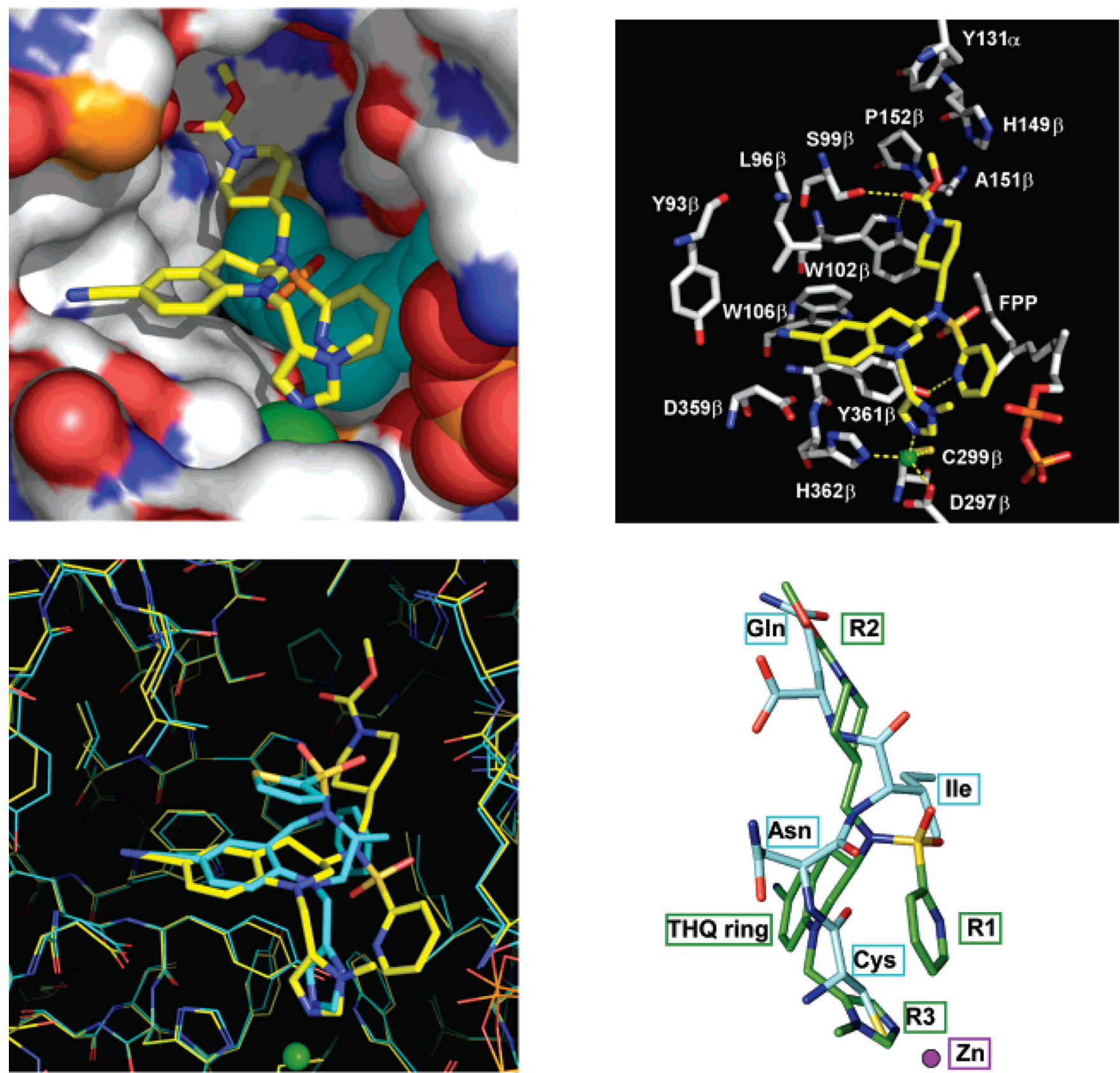

Figure 3.

Experimental binding mode of $\mathbf{1 6 2}$ to rat PFT (top left); molecular surface of rat-PFT (left; C, gray; $\mathrm{N}$, blue; $\mathrm{O}$, red; $\mathrm{S}$, orange), with farnesyl-pyrophosphate (red, orange, and cyan) and $\mathrm{Zn}^{2+}$ (green) in CPK representation and $\mathbf{1 6 2}$ in stick model representation; equivalent all-stick model (top right); comparison of the binding modes of $\mathbf{1 6 2}$ (yellow carbons) and 3-benzyl-1(3H-imidazol-4-ylmethyl)-4-(thiophene-2-sulfonyl)-2,3,4,5-tetrahydro- $1 H$-benzo[ $e][1,4]$ diazepine-7-carbonitrile (cyan carbons, see ref 11 for the structure) to rat PFT (bottom left); superposition of $\mathbf{1 6 2}$ (green carbon atoms) and the C-terminal tetrapeptide fragment of the Rap2a peptide farnesyl acceptor substrate (cyan carbon atoms) as bound in PFT (bottom right; catalytic $\mathrm{Zn}^{2+}$ shown). 

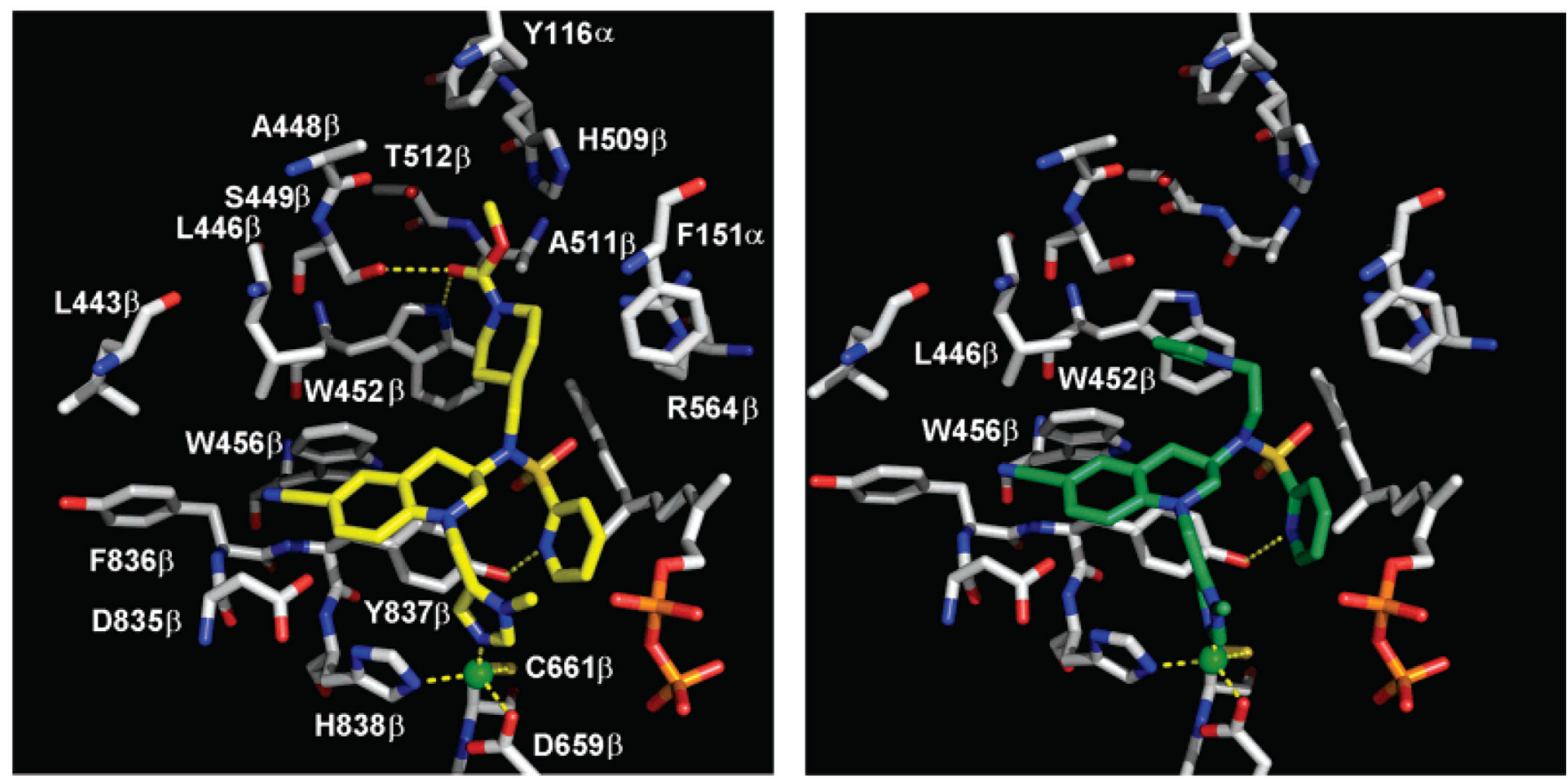

Figure 4.

Predicted binding mode of $\mathbf{1 6 2}$ in the active site of Pf-PFT (top left); molecular surface of PFT (left; C, gray; N, blue; O, red; S, orange), with farnesyl-pyrophosphate (red, orange, and cyan) and $\mathrm{Zn}^{2+}$ (green); predicted binding mode of $\mathbf{5 5}$ in the active site of Pf-PFT (top right). Note how the $\mathrm{R}_{2}$ pyrrole group is wedged between $\mathrm{C} 4$ of the THQ ring and Trp- $452 \beta$ and Trp-456 $\beta$. 

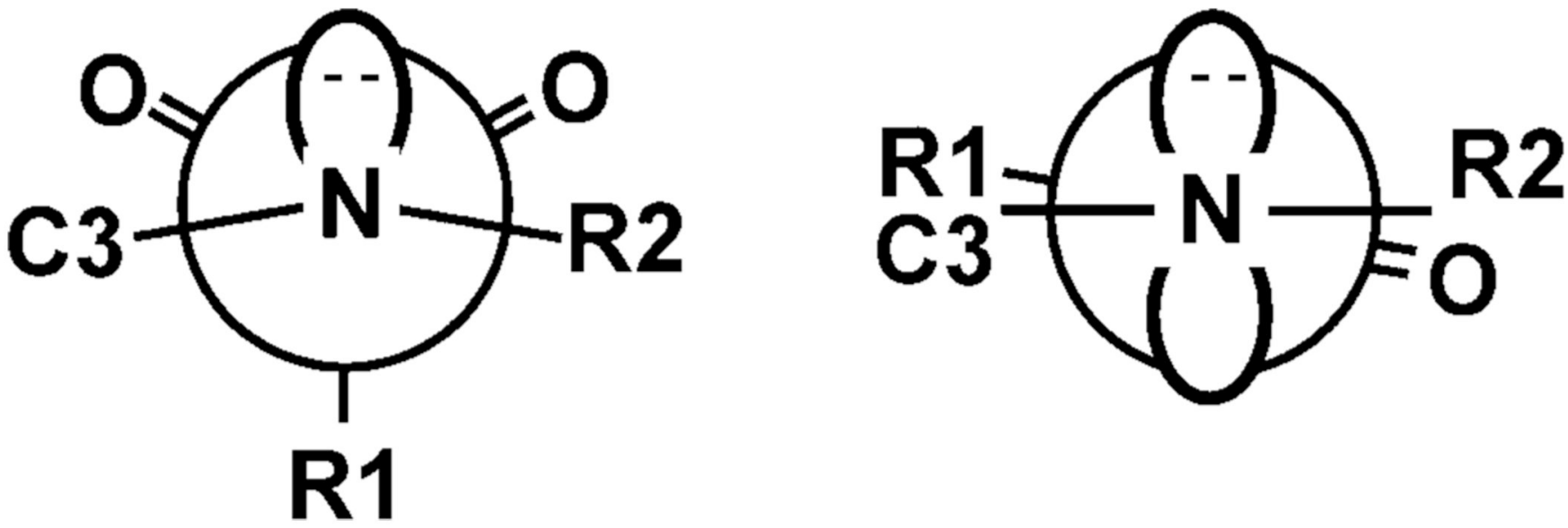

Figure 5.

Comparison of the experimentally observed conformation of THQ PFTIs with a sulfonamide linker (left) and the conformation accessible to the analogous amides (right). The latter projects the $\mathrm{R}_{1}$ group into a sterically poorly accessible area of PFT (not shown). 
<smiles>[Z20]c1cn(C)cn1</smiles>

Scheme 1a.

${ }^{a}$ Reagents and conditions: (i) $\mathrm{R}_{1} \mathrm{SO}_{2} \mathrm{Cl}$, DIPEA, $\mathrm{CH}_{3} \mathrm{CN}$; (ii) 1-methyl- $1 \mathrm{H}$-imidazole-5carboxaldehyde, triethylsilane, 1,2-dichloroethane:trifluoroacetic acid (1:1); (iii) $\mathrm{R}_{2}-\mathrm{Br}$, $\mathrm{Cs}_{2} \mathrm{CO}_{3}$, DMF. 
<smiles>[Y]c1cncn1Cc1ccc(S(=O)(=O)N(CC2CCNCC2)C2Cc3cc(C#N)ccc3N(Cc3cncn3C)C2)nc1</smiles>

Scheme 2a.

${ }^{a}$ Reagents and conditions: (i) $10 \%$ trifluoroacetic acid, $\mathrm{CH}_{2} \mathrm{Cl}_{2}$; (ii) $\mathrm{RCOCl}$ or $\mathrm{ROCOCl}$ or $\mathrm{RNCO}$ or $\mathrm{RSO}_{2} \mathrm{Cl}, \mathrm{CH}_{2} \mathrm{Cl}_{2}$, DIPEA. 
<smiles>[R2]NC1Cc2cc(C#N)ccc2N(Cc2cncn2C)CC1NC(C)C</smiles><smiles>[R7]N([R7])C1Cc2cc(C#N)ccc2N(Cc2cncn2C)C1</smiles>

Scheme 3a.

${ }^{a}$ Reagents and conditions: (i) $\mathrm{Cbz}-\mathrm{Cl}, \mathrm{Et}_{3} \mathrm{~N}, \mathrm{CH}_{3} \mathrm{CN}$; (ii) 1-methyl-1H-imidazole-5carboxaldehyde, triethylsilane, 1,2-dichloroethane:trifluoroacetic acid (1:1); (iii) $10 \% \mathrm{Pd} / \mathrm{C}$, $\mathrm{CH}_{3} \mathrm{OH}$; (iv) $\mathrm{R}_{2}-\mathrm{Br}, \mathrm{Cs}_{2} \mathrm{CO}_{3}$, DMF; (v) $\mathrm{R}_{1} \mathrm{COOH}$, EDC, $\mathrm{HOBt}, \mathrm{Et}_{3} \mathrm{~N}$, DMF, or $\mathrm{R}_{1} \mathrm{COCl}$, $\mathrm{Et}_{3} \mathrm{~N}, \mathrm{CH}_{2} \mathrm{Cl}_{2}$ or $\mathrm{R}_{1} \mathrm{SO}_{2} \mathrm{Cl}, \mathrm{Et}_{3} \mathrm{~N}, \mathrm{CH}_{2} \mathrm{Cl}_{2}$. 
<smiles>[R7]N(C1Cc2cc(C#N)ccc2N(Cc2cn([Tl])cn2)C1)S([R])(=O)=O</smiles>

Scheme 4a.

${ }^{a}$ Reagents and conditions: (i) $3 \mathrm{H}$-imidazole-4-carboxaldehyde, triethylsilane, 1,2dichloroethane/trifluoroacetic acid (1:1); (ii) trityl chloride, DIPEA, DMF; (iii) $\mathrm{R}_{2}-\mathrm{Br}$, $\mathrm{Cs}_{2} \mathrm{CO}_{3}$, DMF; (iv) $\mathrm{CH}_{2} \mathrm{Cl}_{2}$, trifluoroacetic acid. 
<smiles>COC(=O)N1CCC(CN(C2CCN(S(=O)(=O)c3ccccn3)CC2)S(=O)(=O)c2cccc(C(C)C)n2)CC1</smiles>

Scheme 5a.

${ }^{a}$ Reagents and conditions: (i) $\mathrm{R}_{2}-\mathrm{Br}, \mathrm{Cs}_{2} \mathrm{CO}_{3}$, DMF; (ii) 3-methyl-3H-imidazole-4-sulfonyl chloride, DMAP, $\mathrm{CH}_{3} \mathrm{CN}$. 
<smiles>CC#CC1CNc2ccc(-c3ccccc3)cc2CC1NC(=O)OCc1ccccc1</smiles><smiles>[R1]S(=O)(=O)N[C@H]1Cc2cc(-c3ccccc3)ccc2N(Cc2cncn2C)C1</smiles><smiles>[R]N(C1Cc2cc(-c3ccccc3)ccc2N(Cc2cncn2C)C1)S([R])(=O)=O</smiles>

Scheme 6a.

${ }^{a}$ Reagents and conditions: (i) BOC-anhydride, $\mathrm{K}_{2} \mathrm{CO}_{3}$, dioxane-water (4:1); (ii) phenylboronic acid, $\mathrm{Ba}(\mathrm{OH})_{2}$, tetrakis triphenylphosphine palladium, DME-water (5:1); (iii) $20 \%$ trifluoroacetic acid, $\mathrm{CH}_{2} \mathrm{Cl}_{2}$; (iv) $\mathrm{R}_{1} \mathrm{SO}_{2} \mathrm{Cl}$, DIPEA, $\mathrm{CH}_{2} \mathrm{Cl}_{2}$; (v) 1-methyl-1H-imidazole-5carboxaldehyde, triethylsilane, 1,2-dichloroethane/trifluoroacetic acid (1:1); (vi) $\mathrm{R}_{2}-\mathrm{Br}$, $\mathrm{Cs}_{2} \mathrm{CO}_{3}$, DMF. 
<smiles>[R]NC(=O)c1ccc2c(c1)CC(NS(=O)(=O)c1cn(C)cn1)CN2Cc1cncn1C</smiles>

Scheme 7a.

${ }^{a}$ Reagents and conditions: (i) concd $\mathrm{HCl}, 80{ }^{\circ} \mathrm{C}$; (ii) $\mathrm{H}_{2} \mathrm{SO}_{4}, \mathrm{R}_{4}-\mathrm{OH}$; (iii) $\mathrm{R}_{4}-\mathrm{NH}_{2}, \mathrm{EDC}$, DMAP, DMF; (iv) alkyl bromide, $\mathrm{Cs}_{2} \mathrm{CO}_{3}$, DMF. 
<smiles></smiles><smiles>COC(=O)N1CCC(CN(C2Cc3cc(C#N)ccc3N(Cc3cnc[nH]3)C2)S(=O)(=O)c2ccccn2)CC1</smiles>

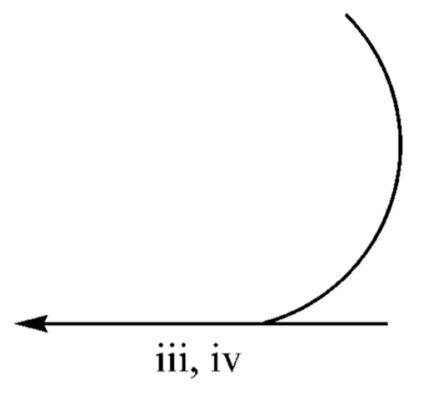<smiles>COC(=O)N1CCC(CN(C2CNc3ccc(C#N)cc3C2)S(=O)(=O)c2ccccn2)CC1</smiles>

Scheme 8a.

${ }^{a}$ Reagents and conditions: (i) trityl chloride, $\mathrm{Et}_{3} \mathrm{~N}$, DMF; (ii) $\mathrm{MeMgBr}$, $\mathrm{THF}, 0^{\circ} \mathrm{C}$; (iii) $\mathrm{MsCl}$, $\mathrm{CH}_{3} \mathrm{CN}, 60^{\circ} \mathrm{C}$; (iv) trifluoroacetic acid, $\mathrm{CH}_{2} \mathrm{Cl}_{2}$. 


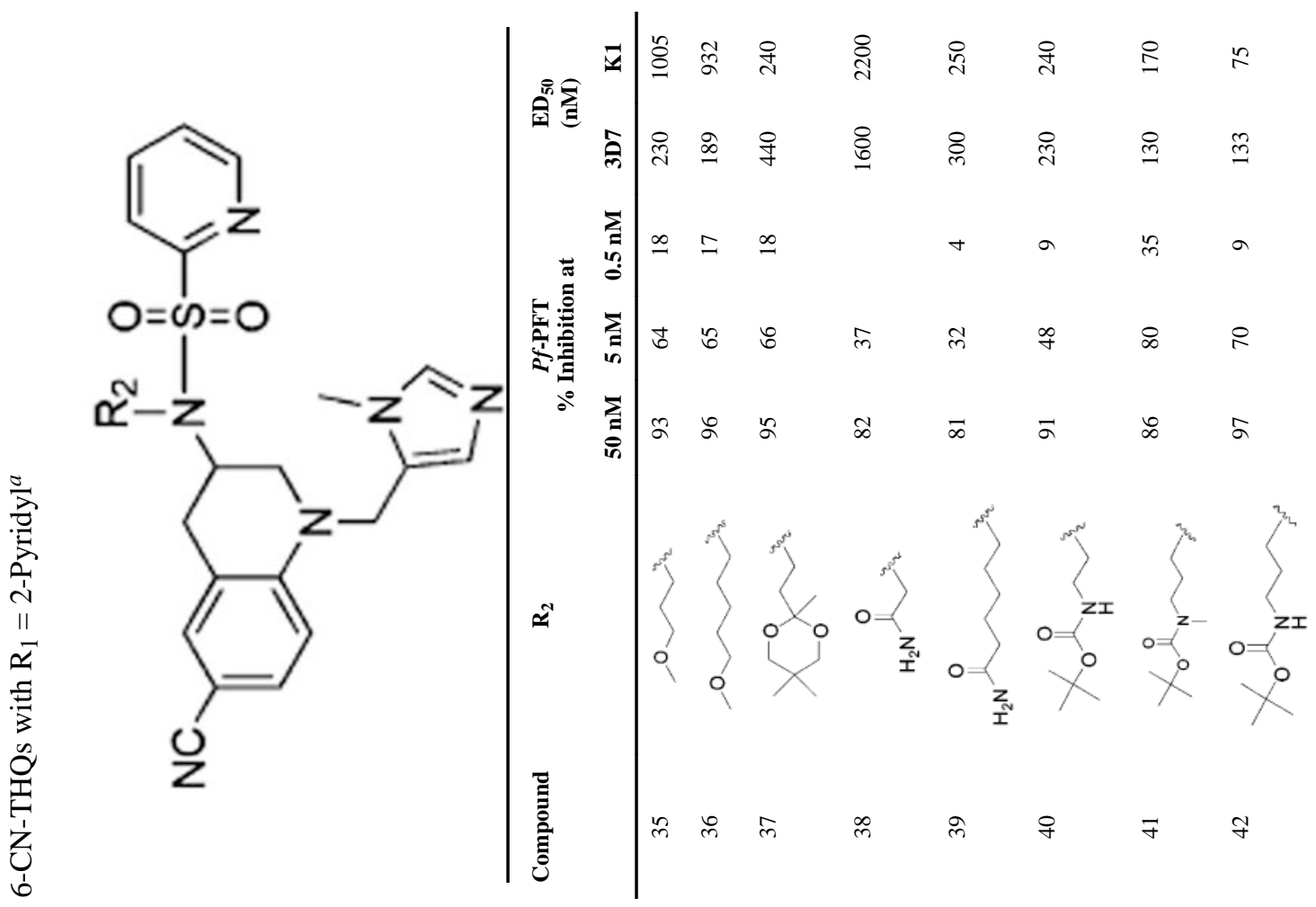




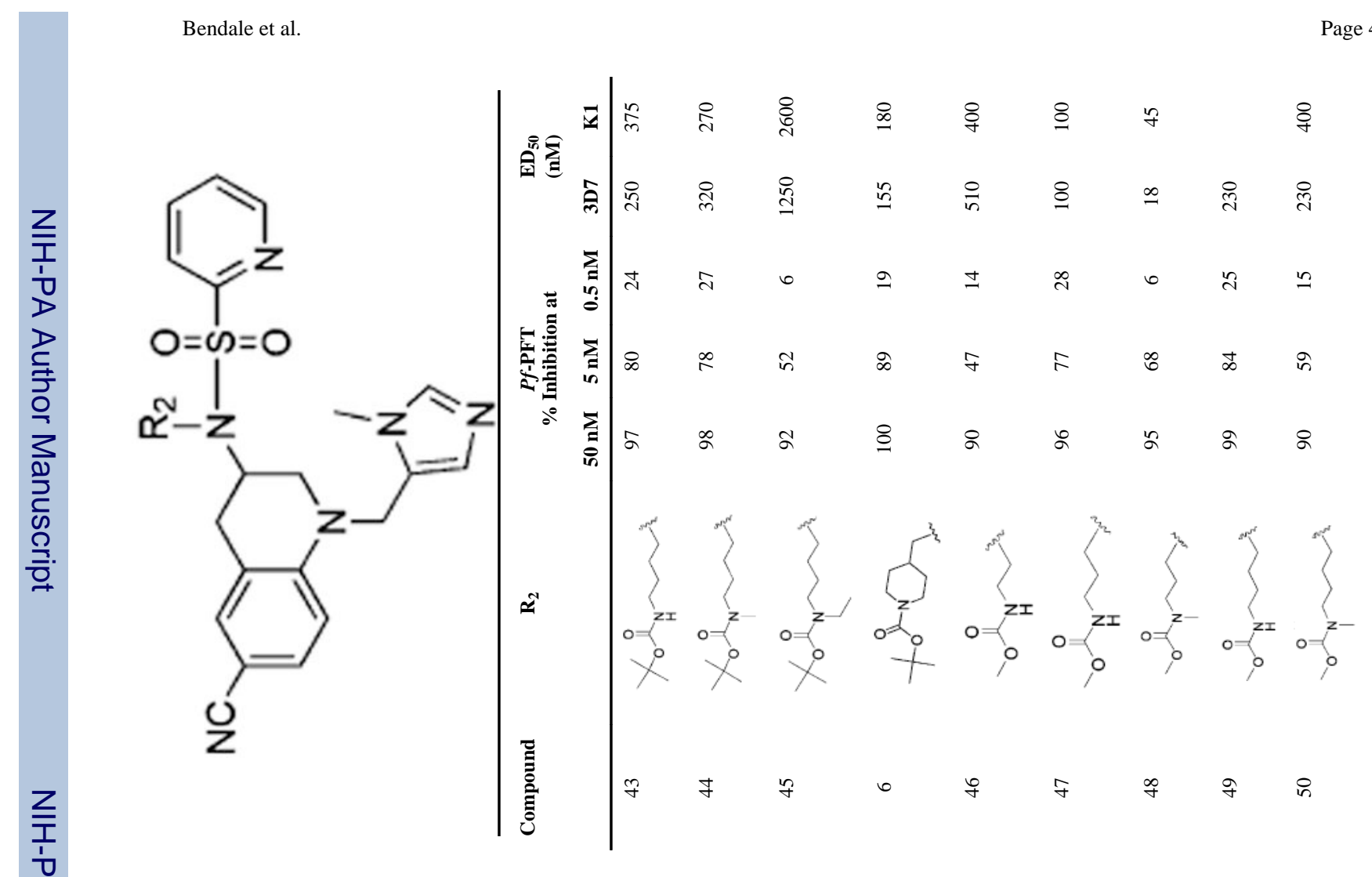




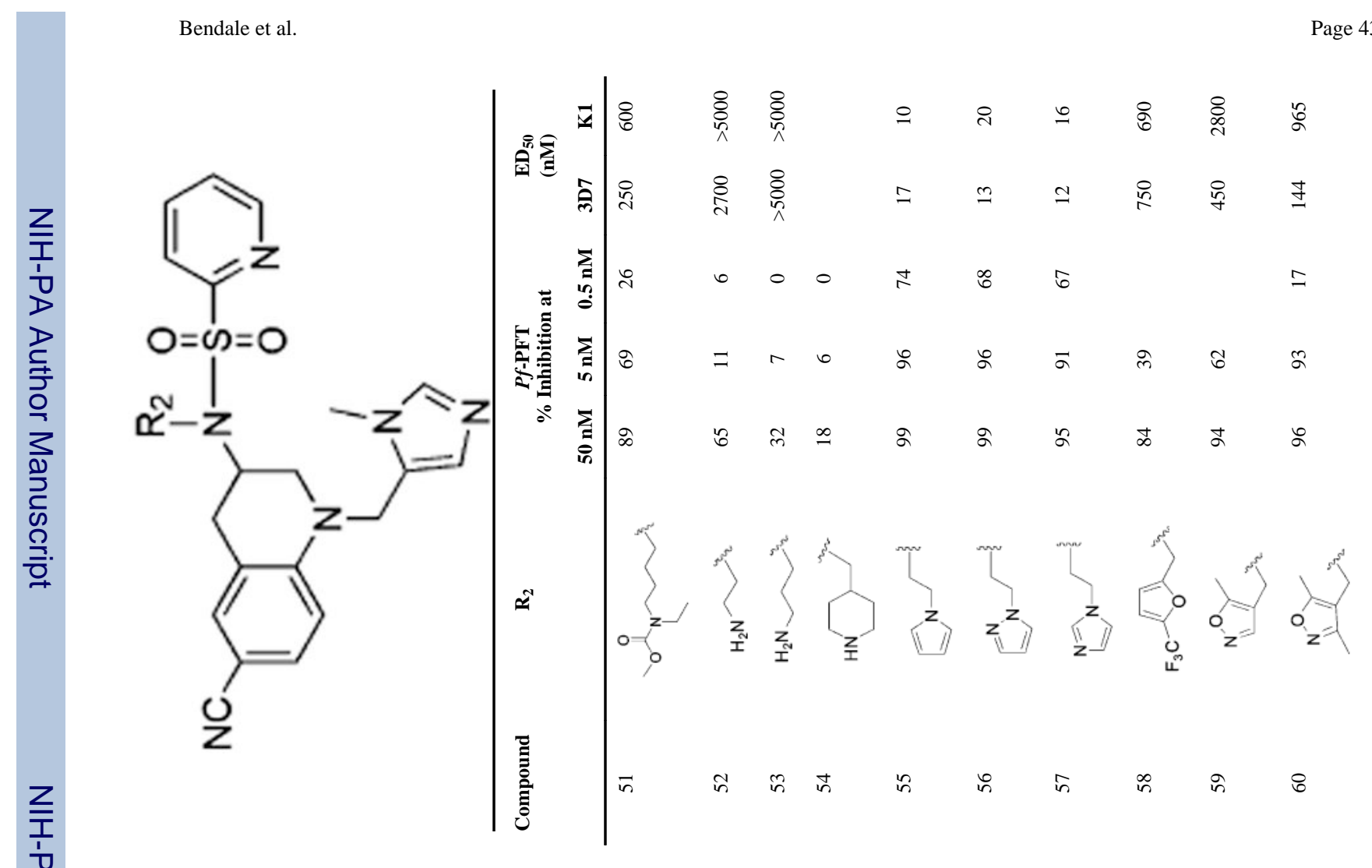




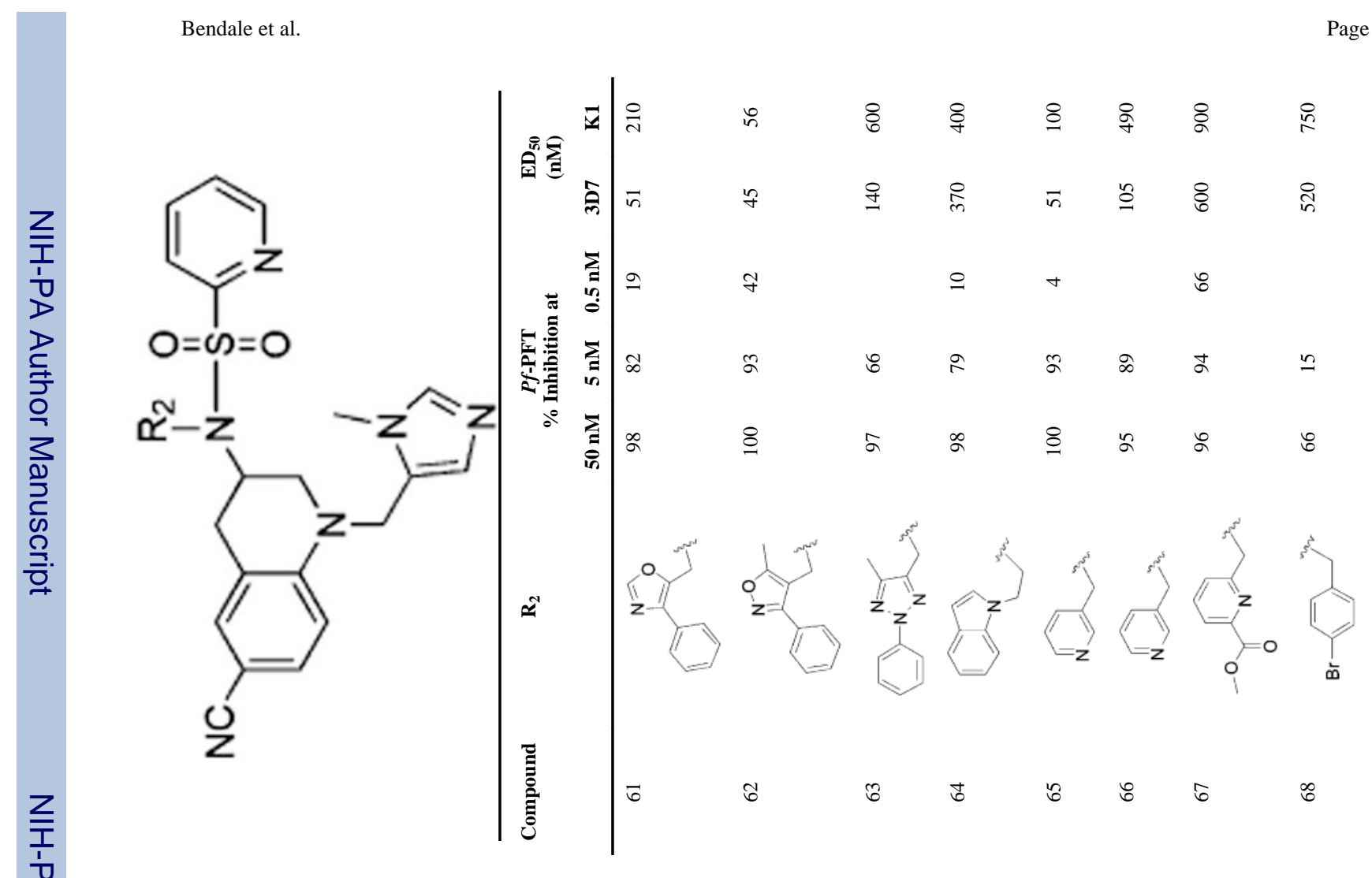




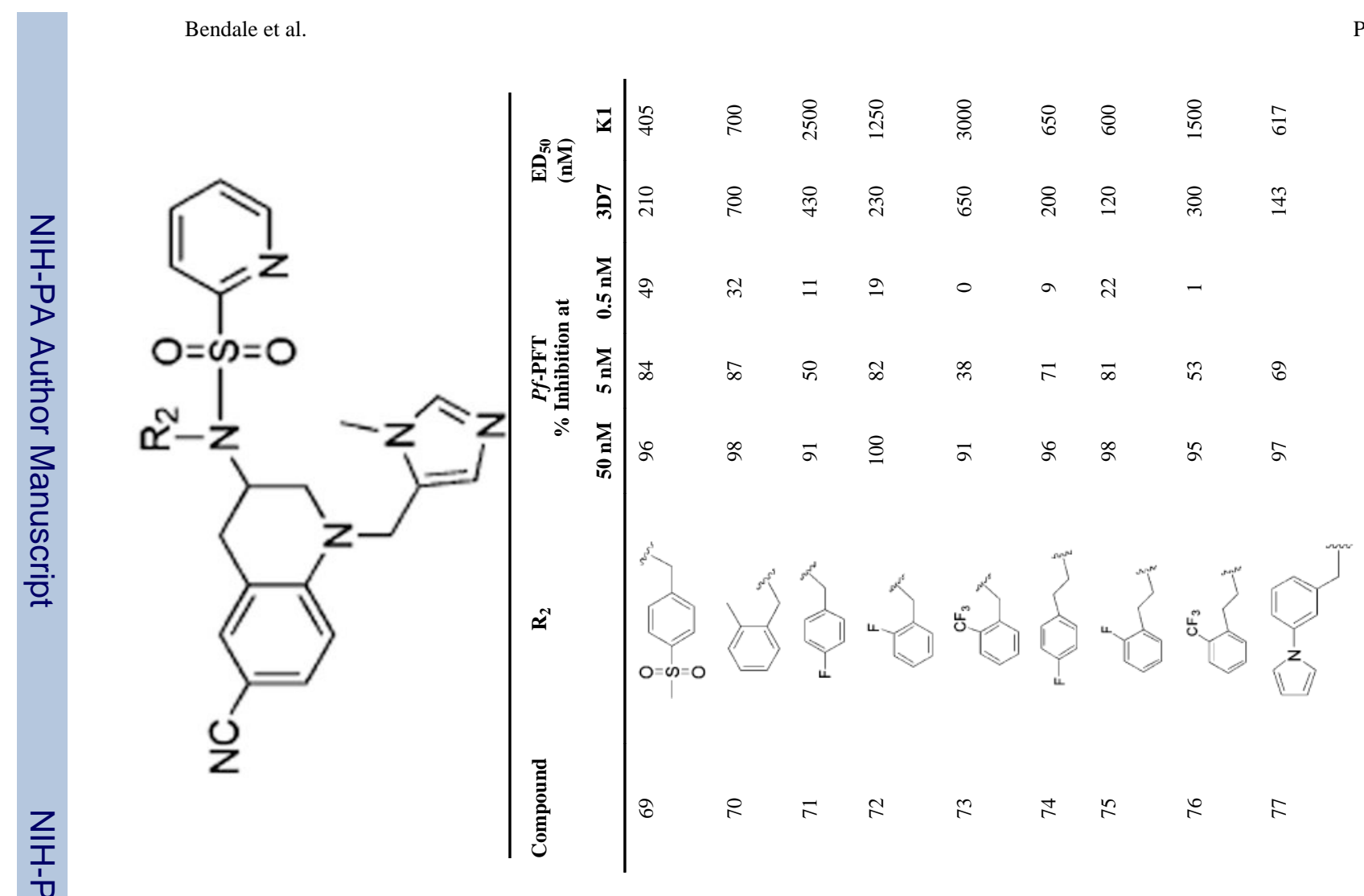




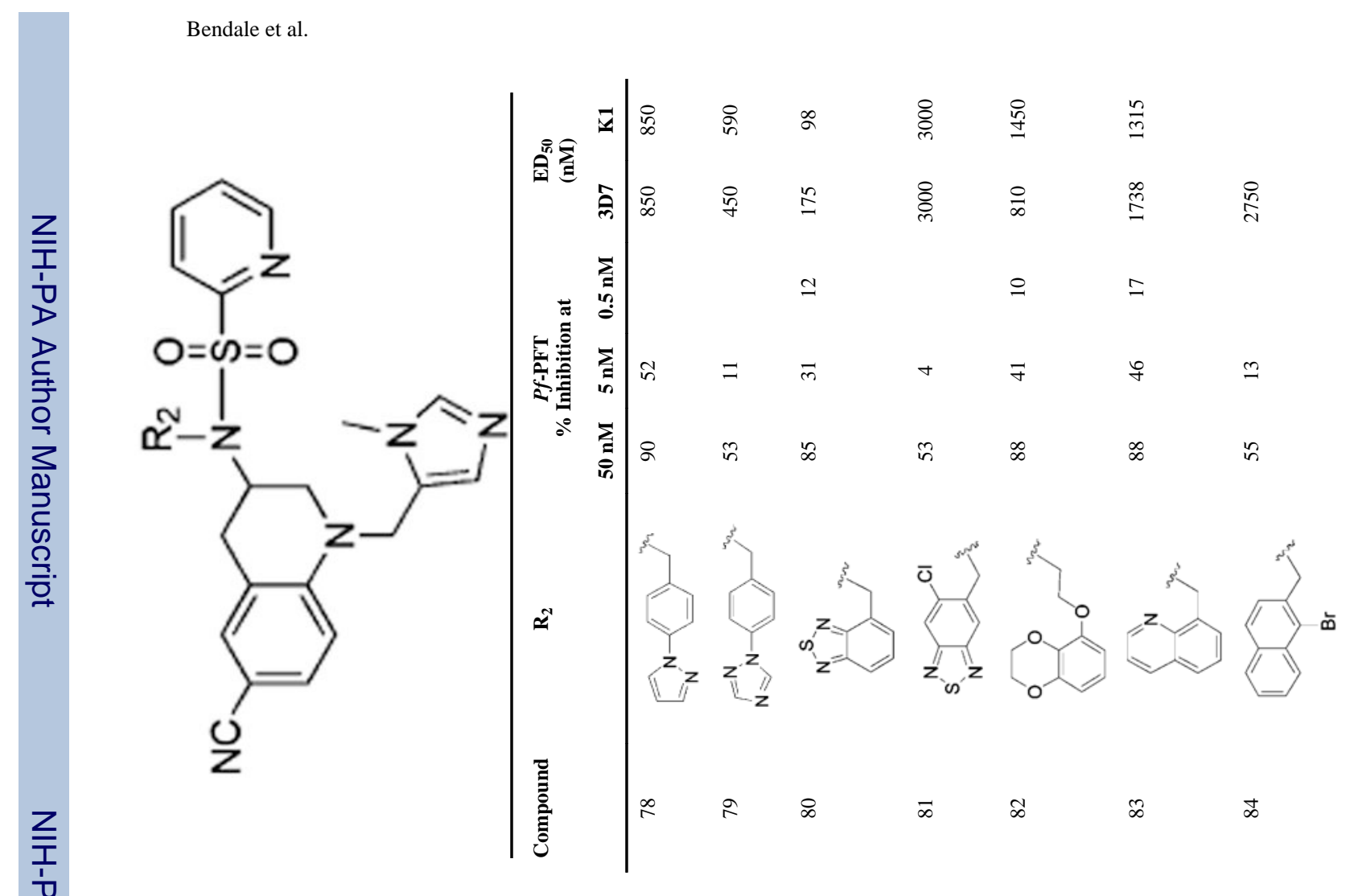




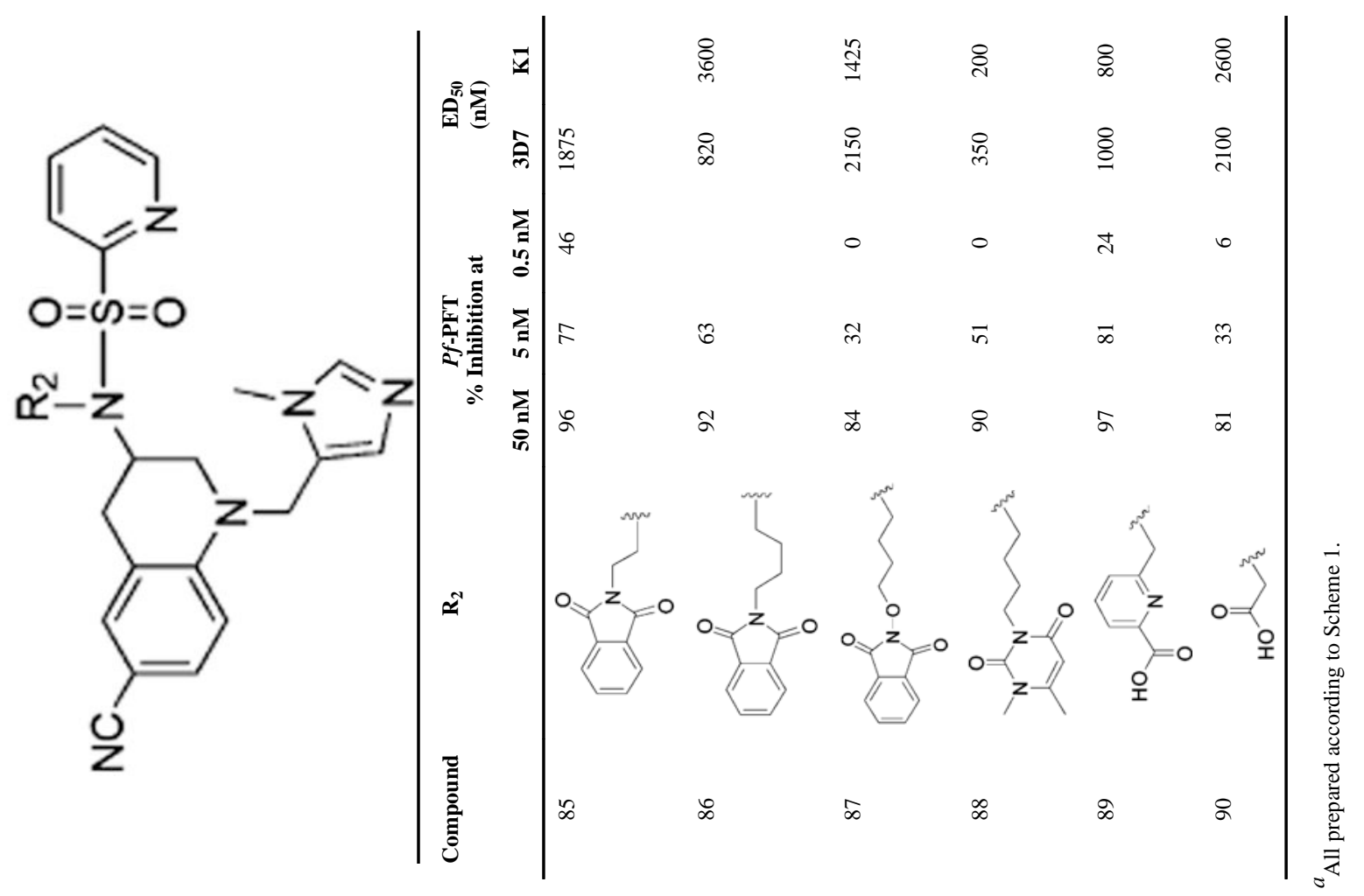

J Med Chem. Author manuscript; available in PMC 2010 June 30. 


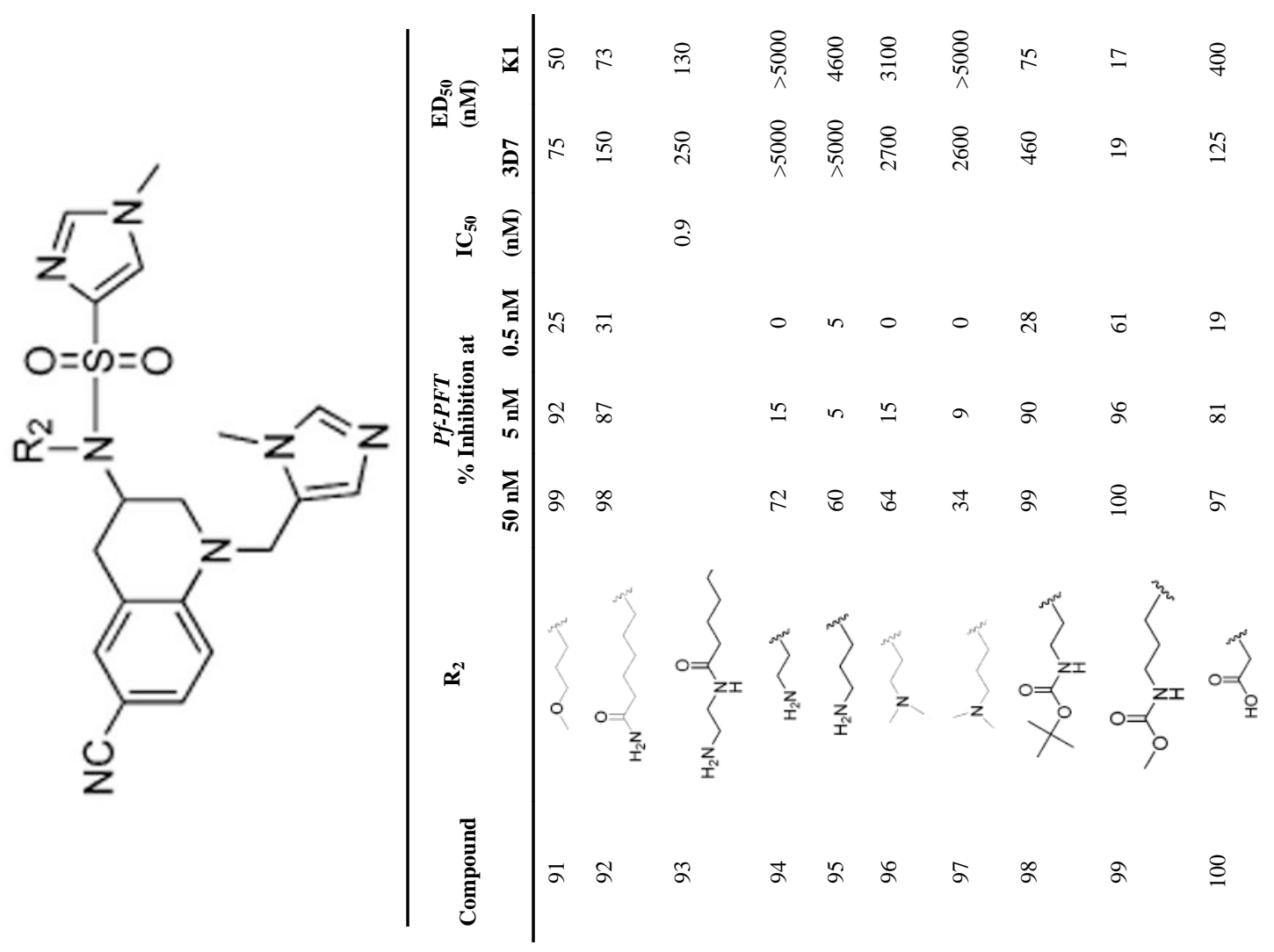

J Med Chem. Author manuscript; available in PMC 2010 June 30. 


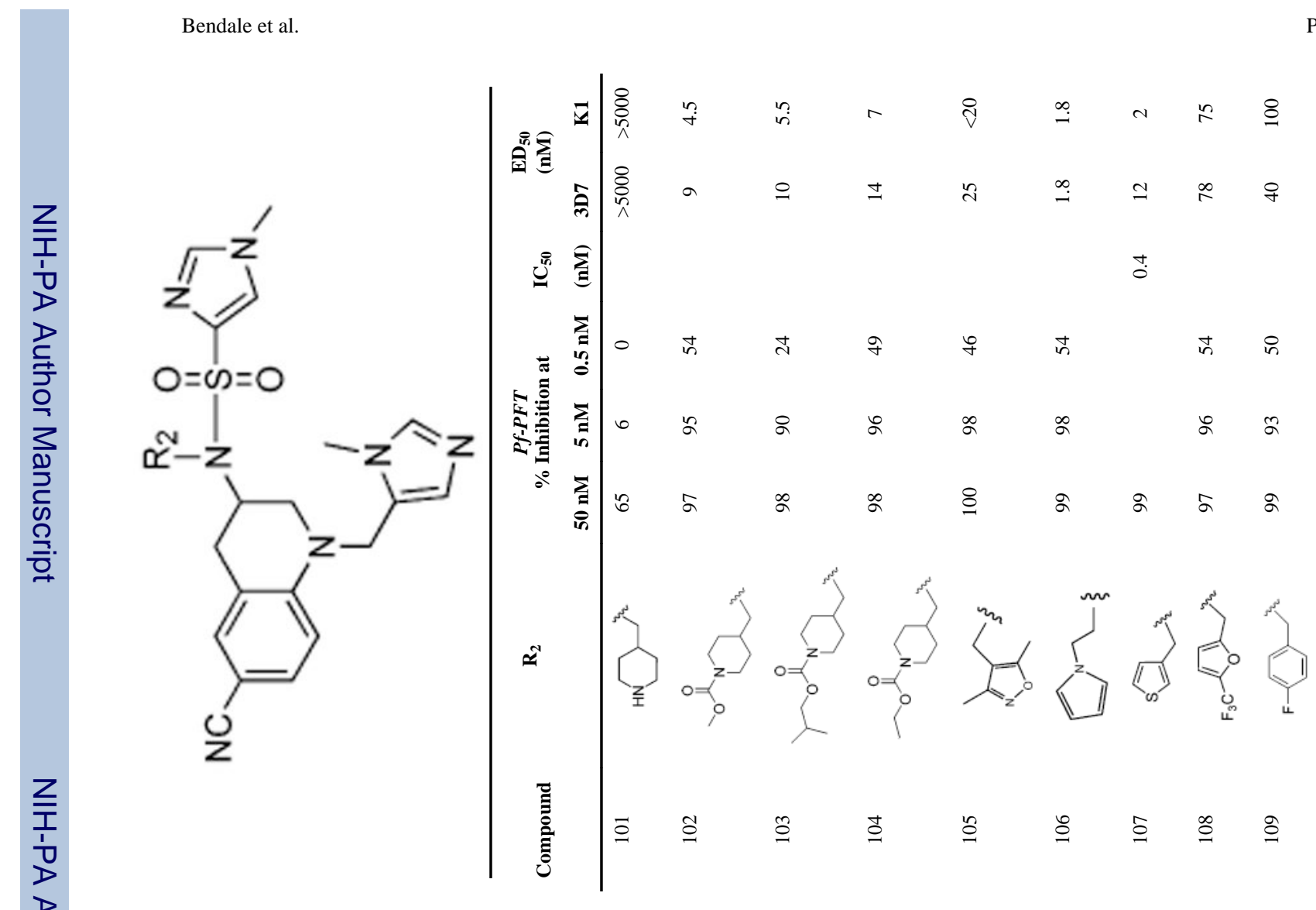




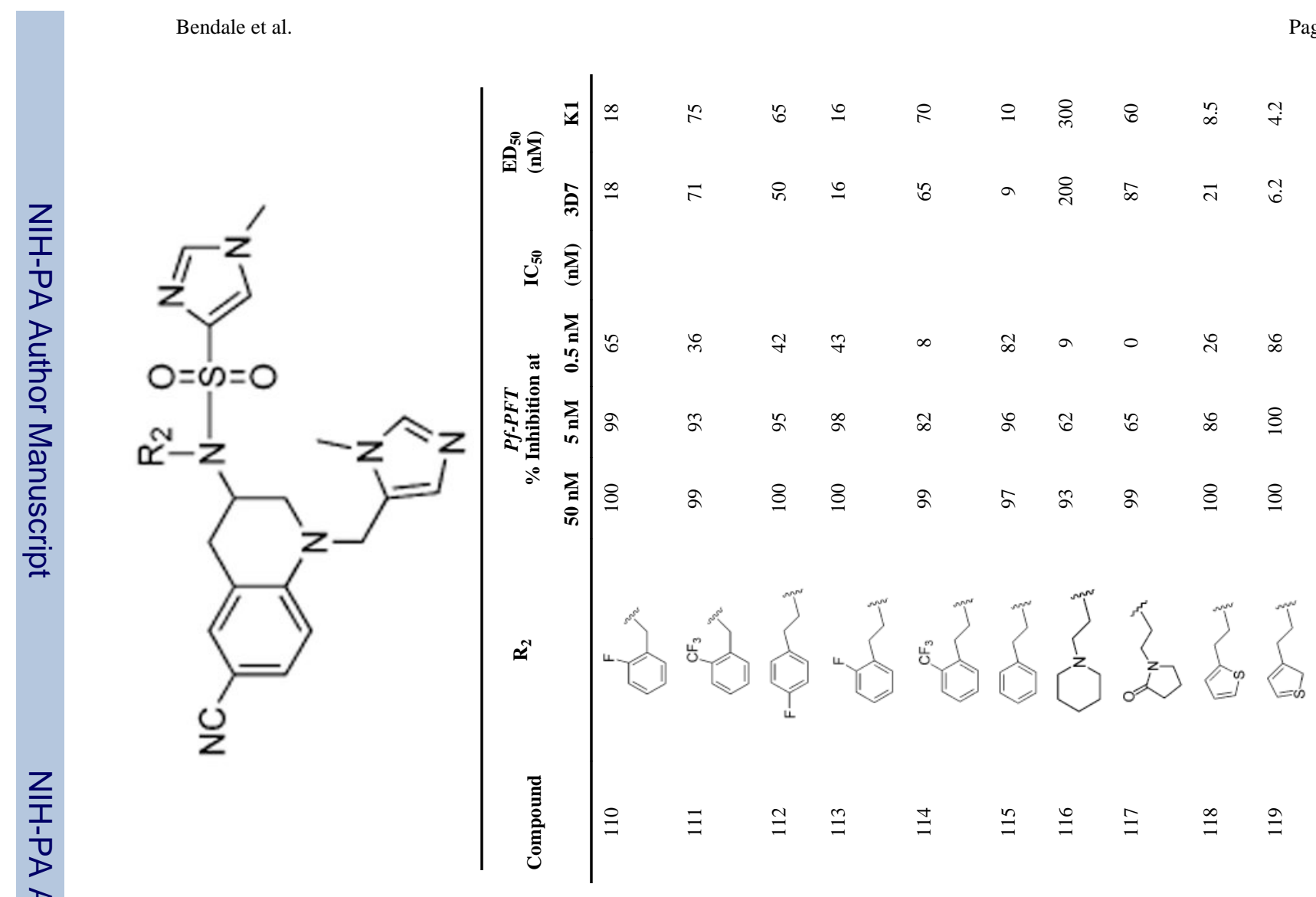




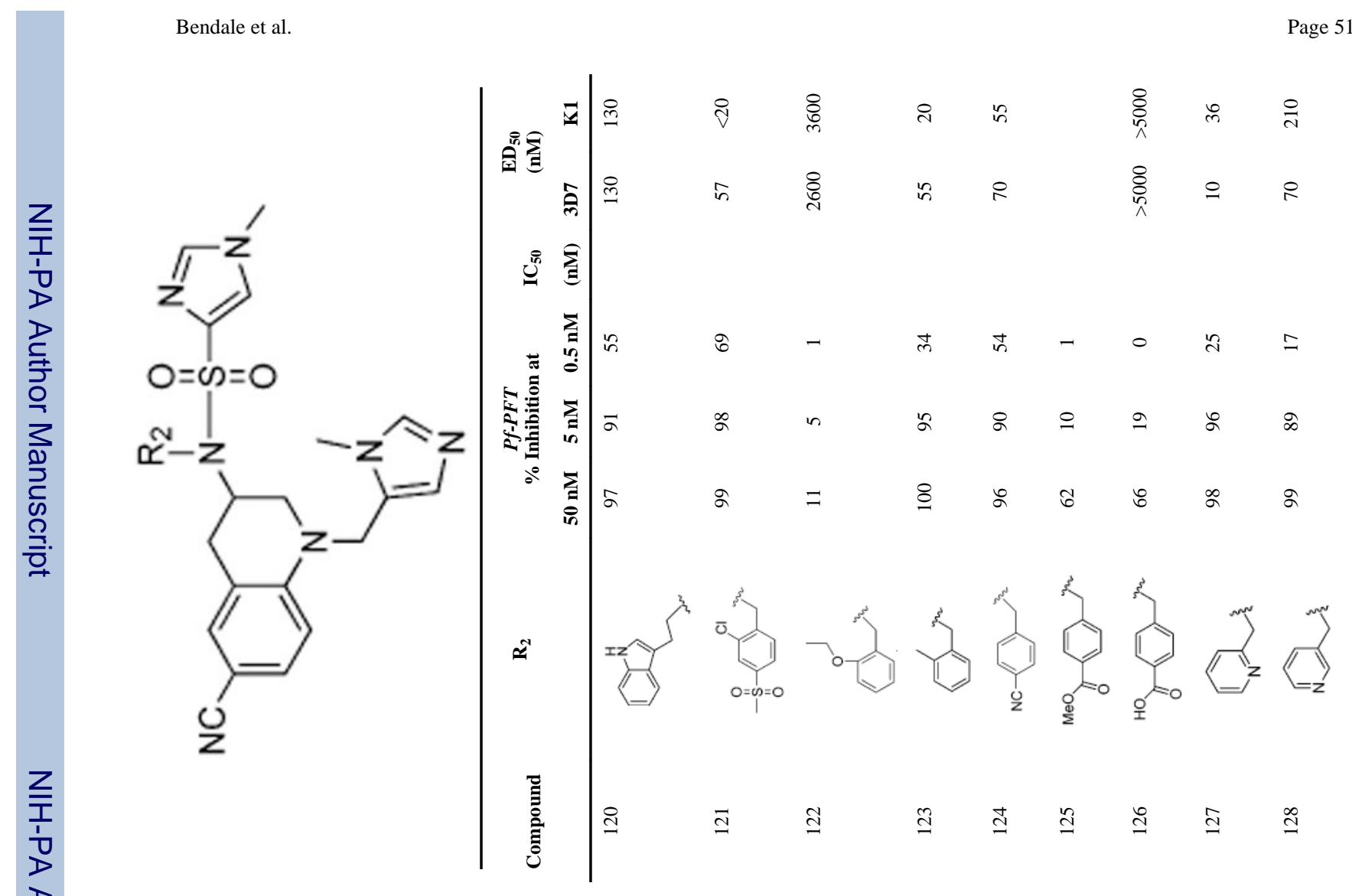




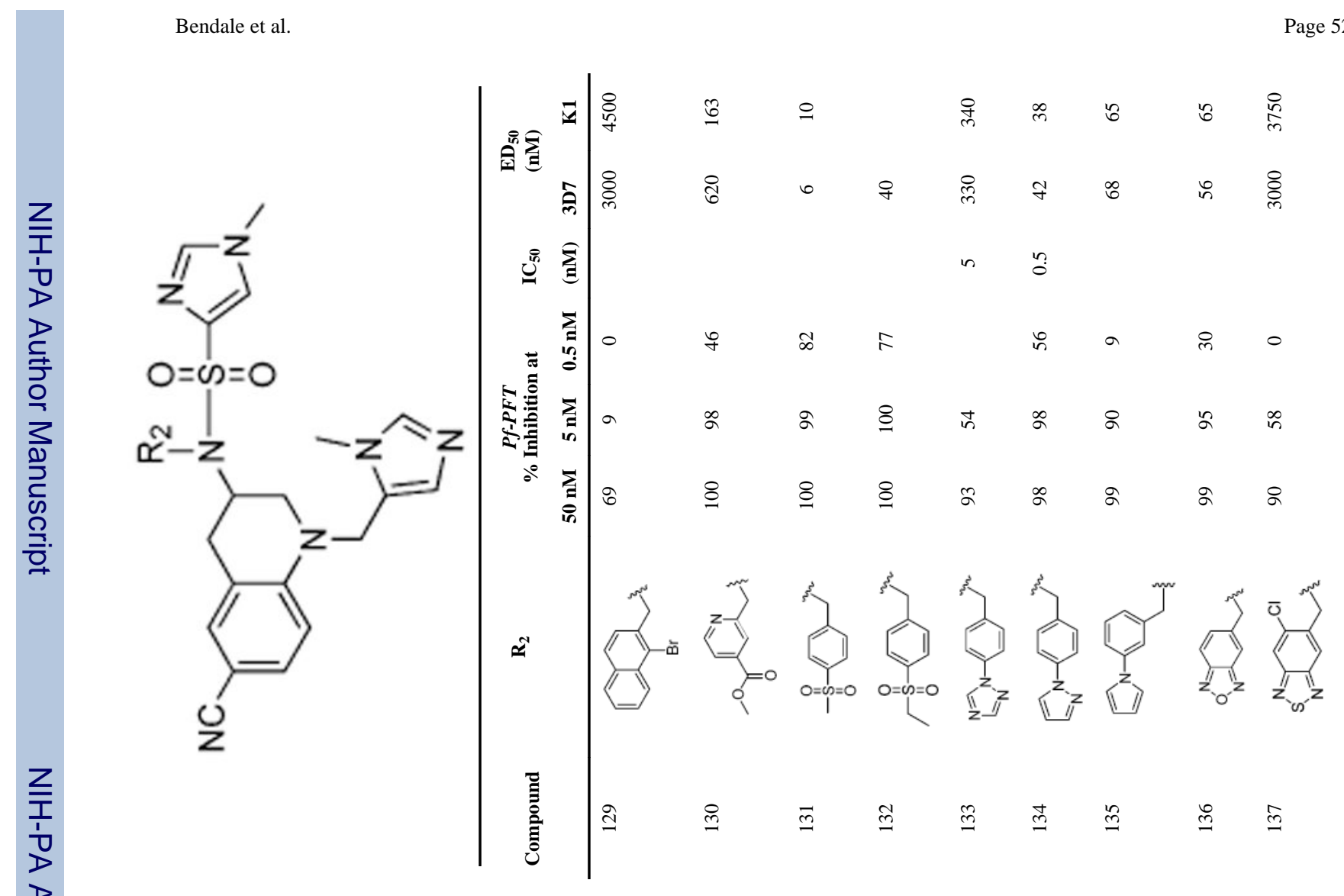




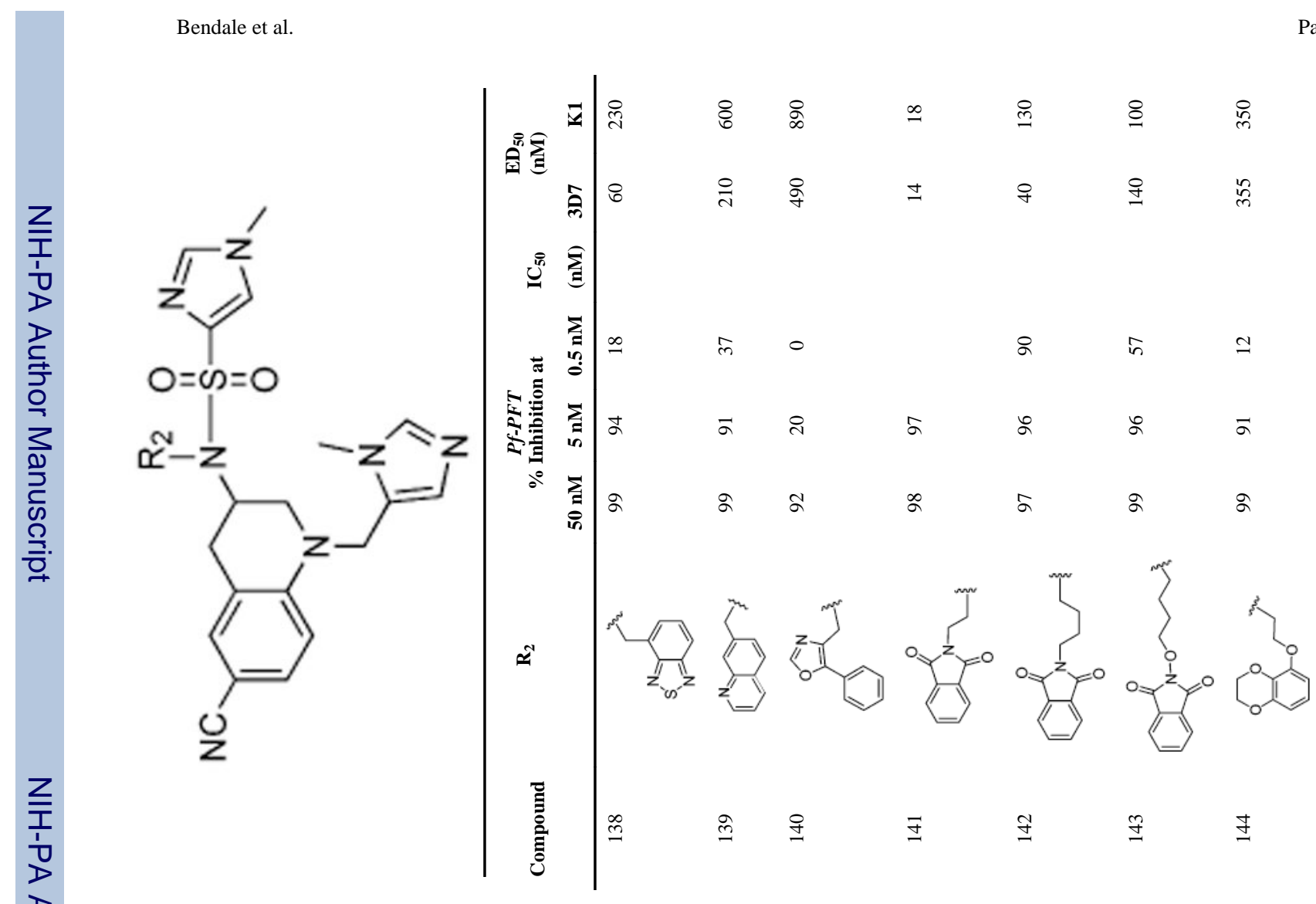




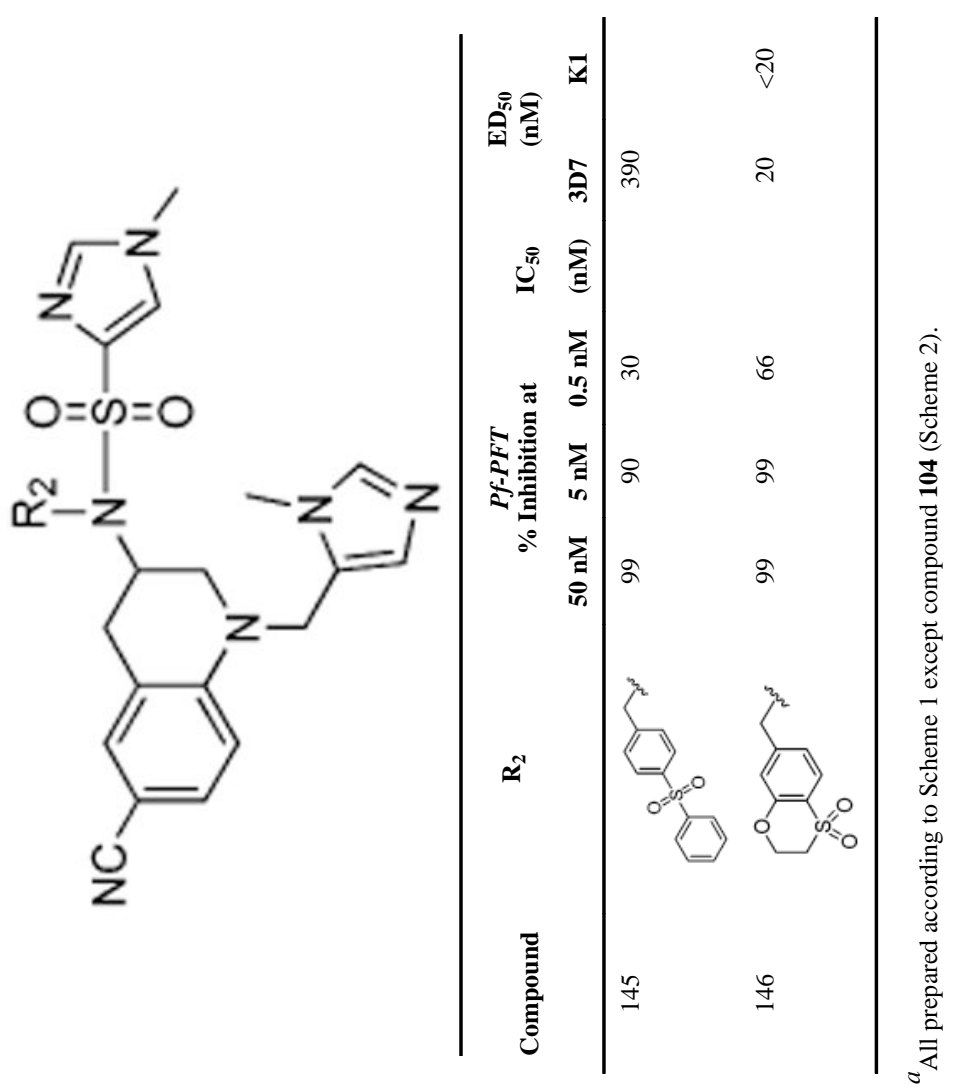

J Med Chem. Author manuscript; available in PMC 2010 June 30. 


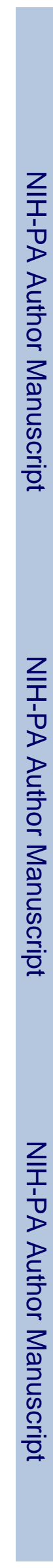

Bendale et al.

Page 56

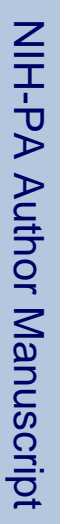

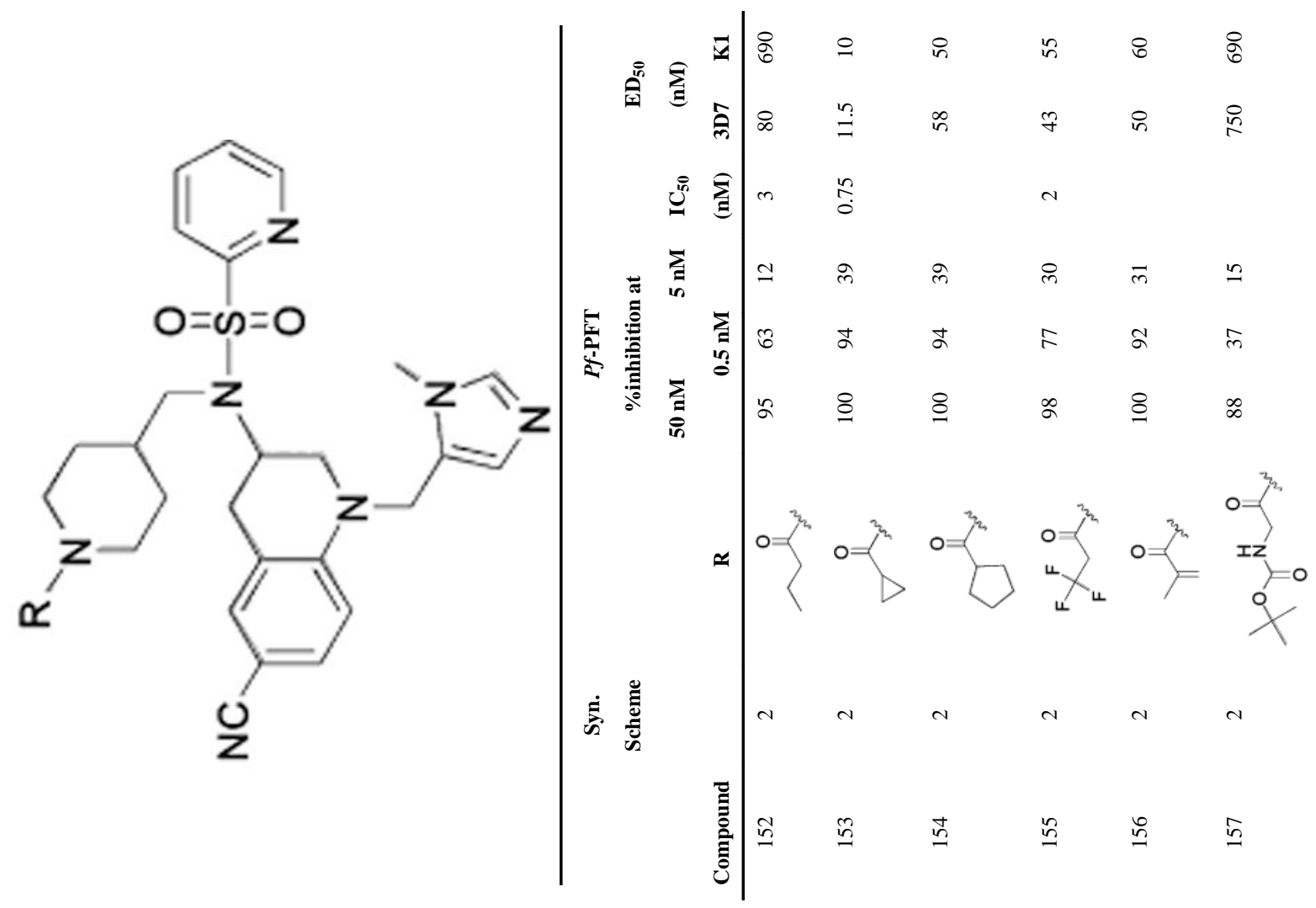




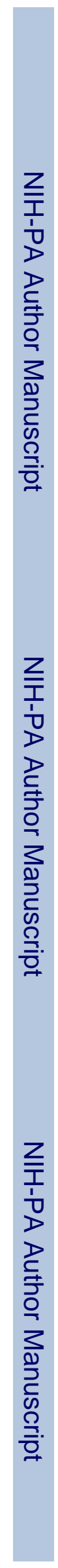

Bendale et al.

Page 57

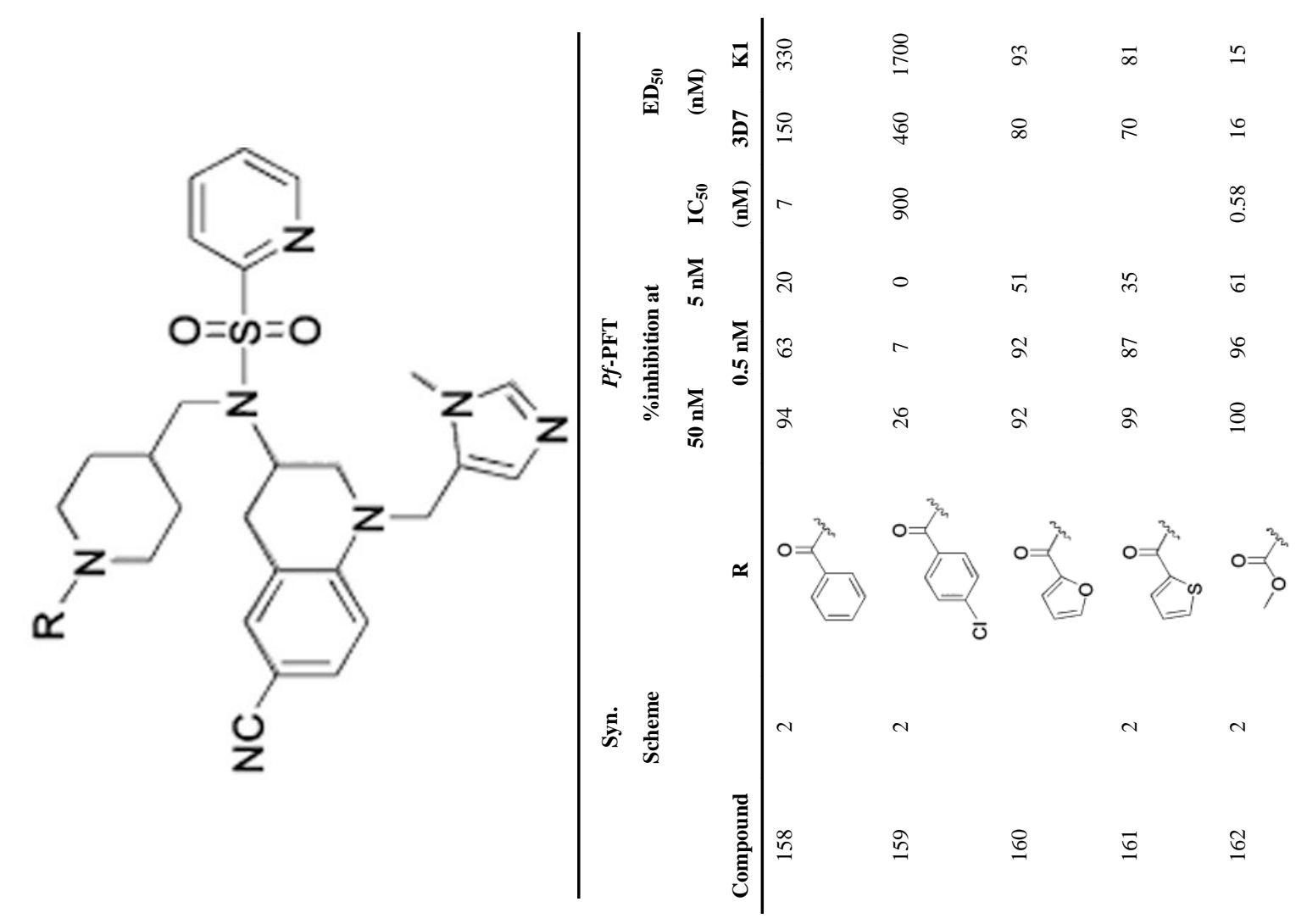

J Med Chem. Author manuscript; available in PMC 2010 June 30. 


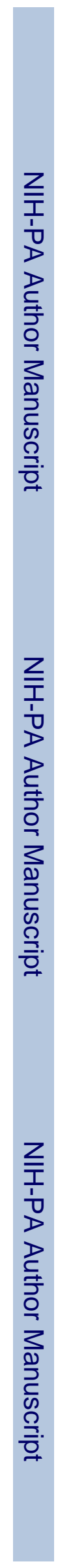

Bendale et al.

Page 58

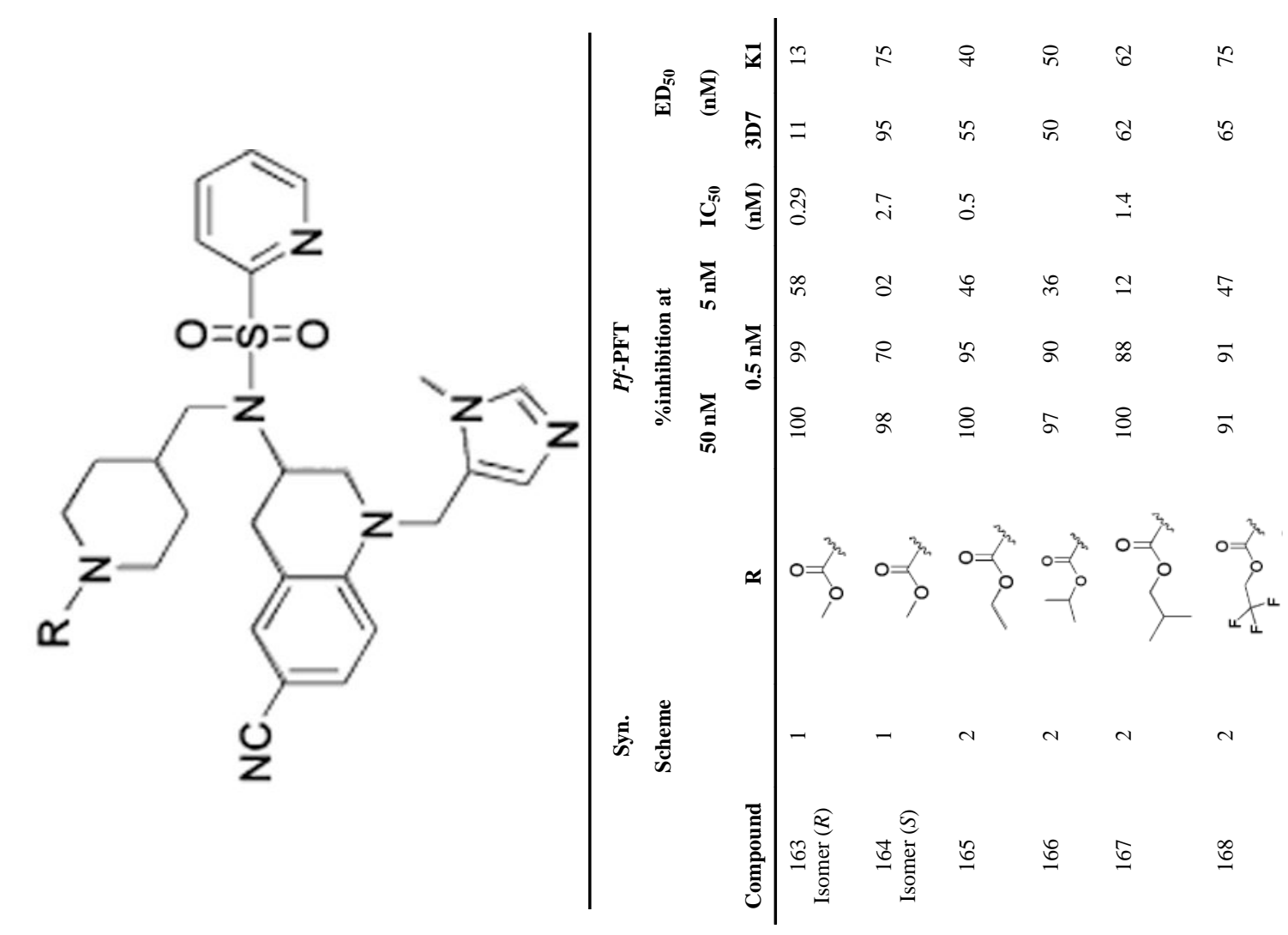

J Med Chem. Author manuscript; available in PMC 2010 June 30. 

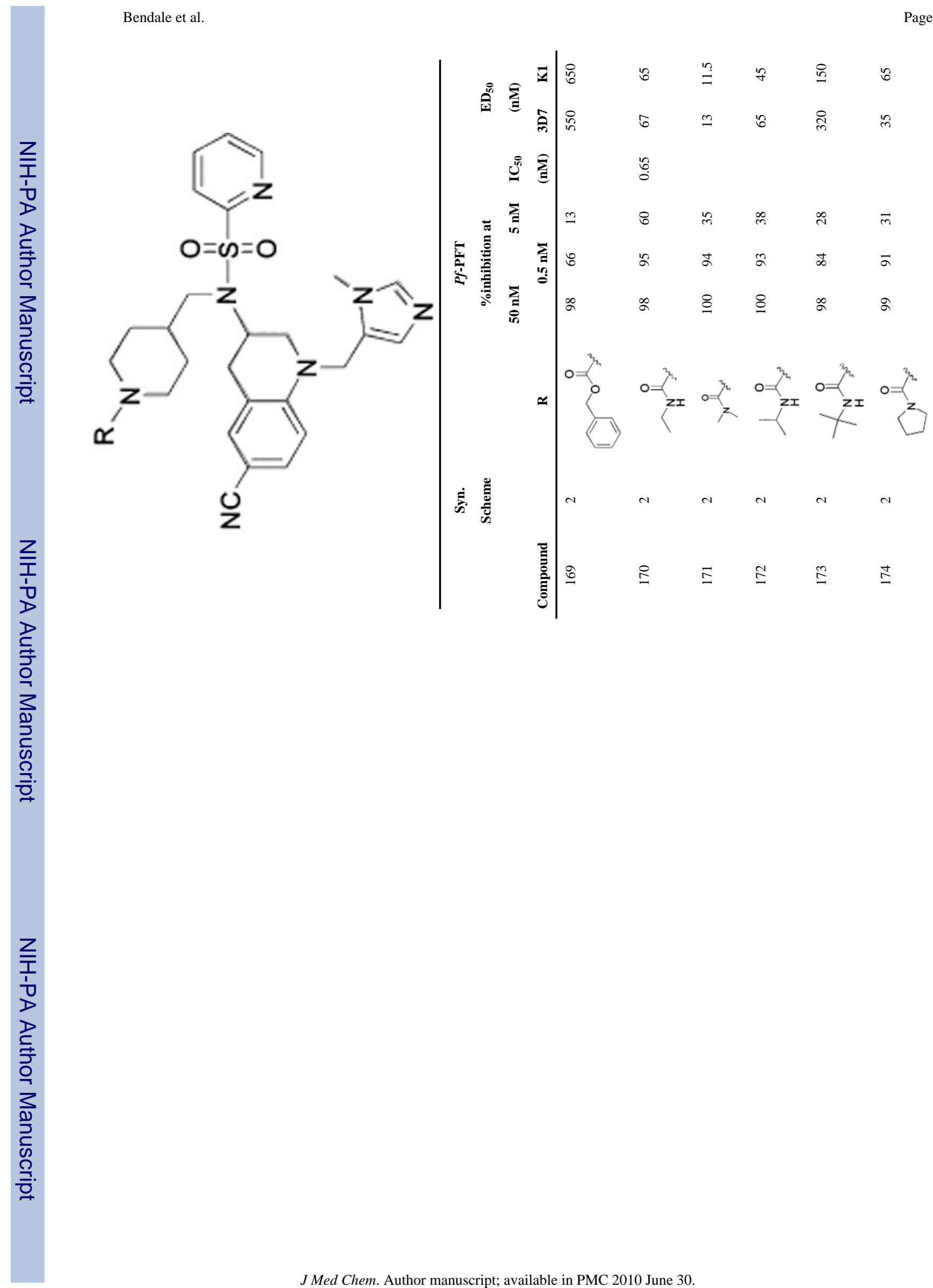


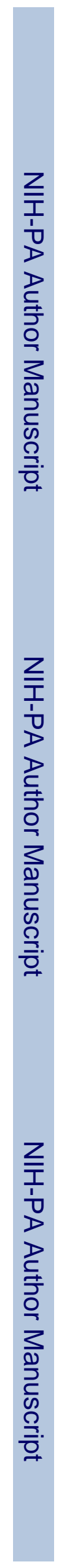

Bendale et al.

Page 60

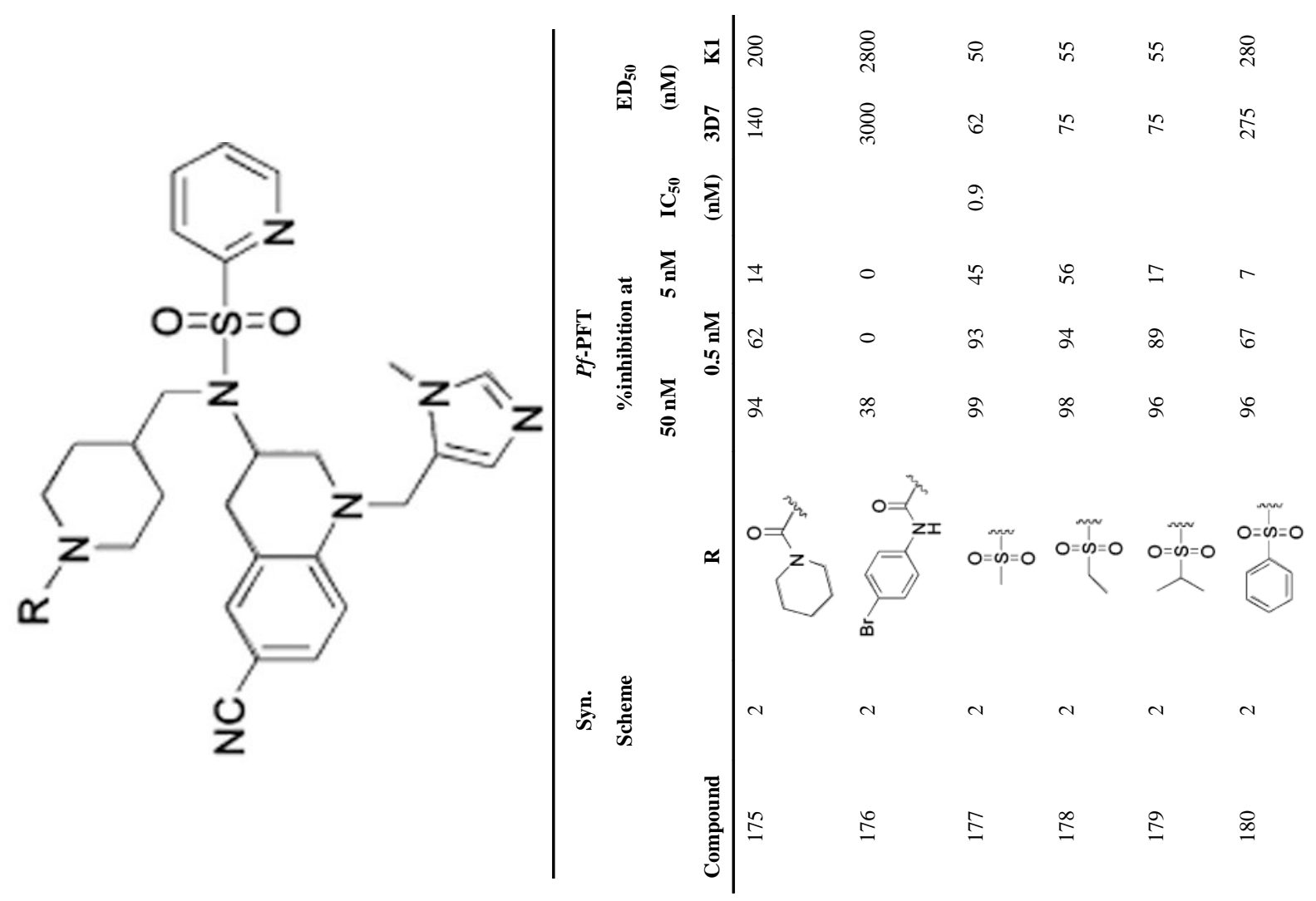

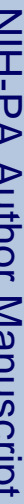

J Med Chem. Author manuscript; available in PMC 2010 June 30. 

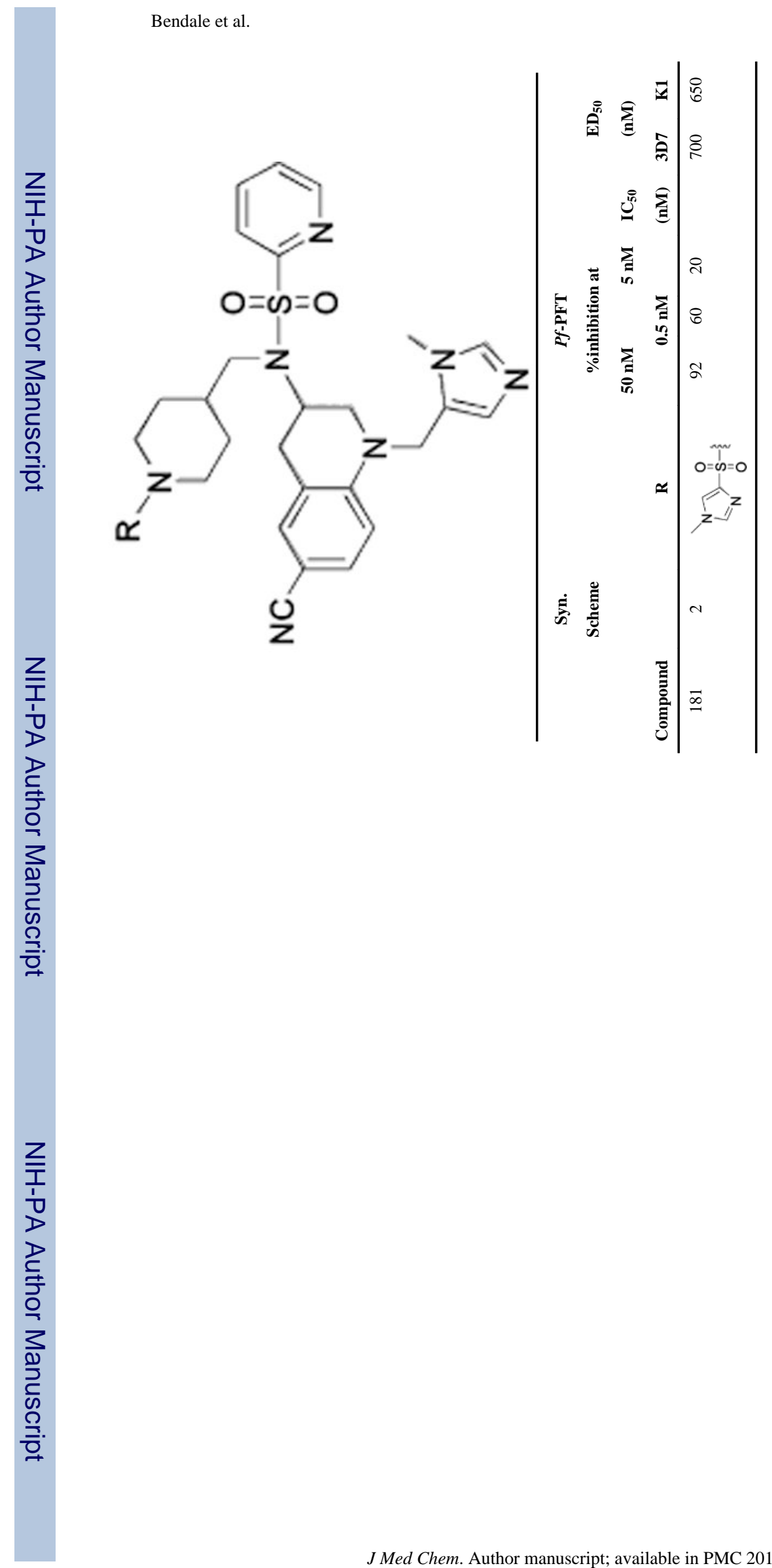

J Med Chem. Author manuscript; available in PMC 2010 June 30. 


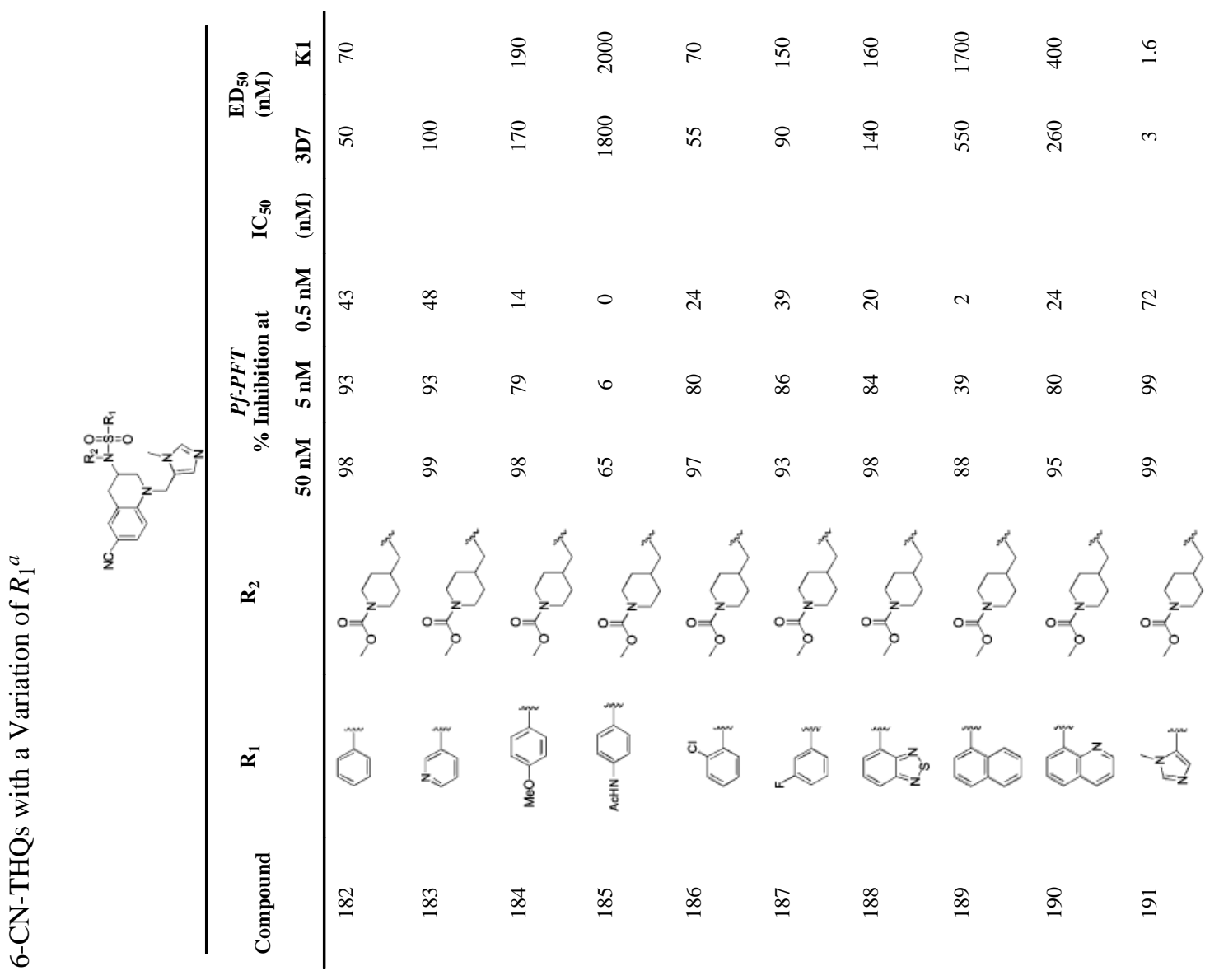




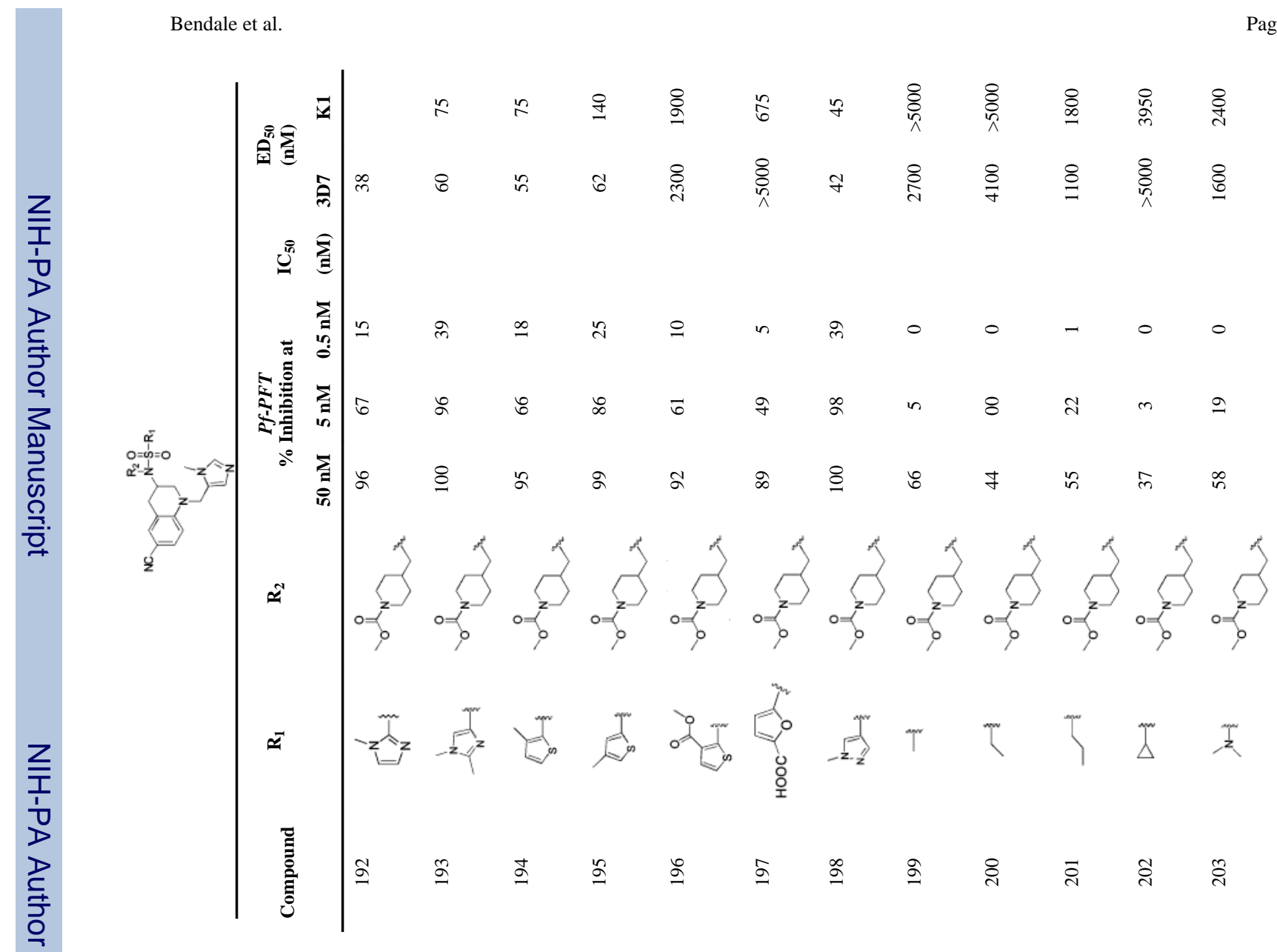




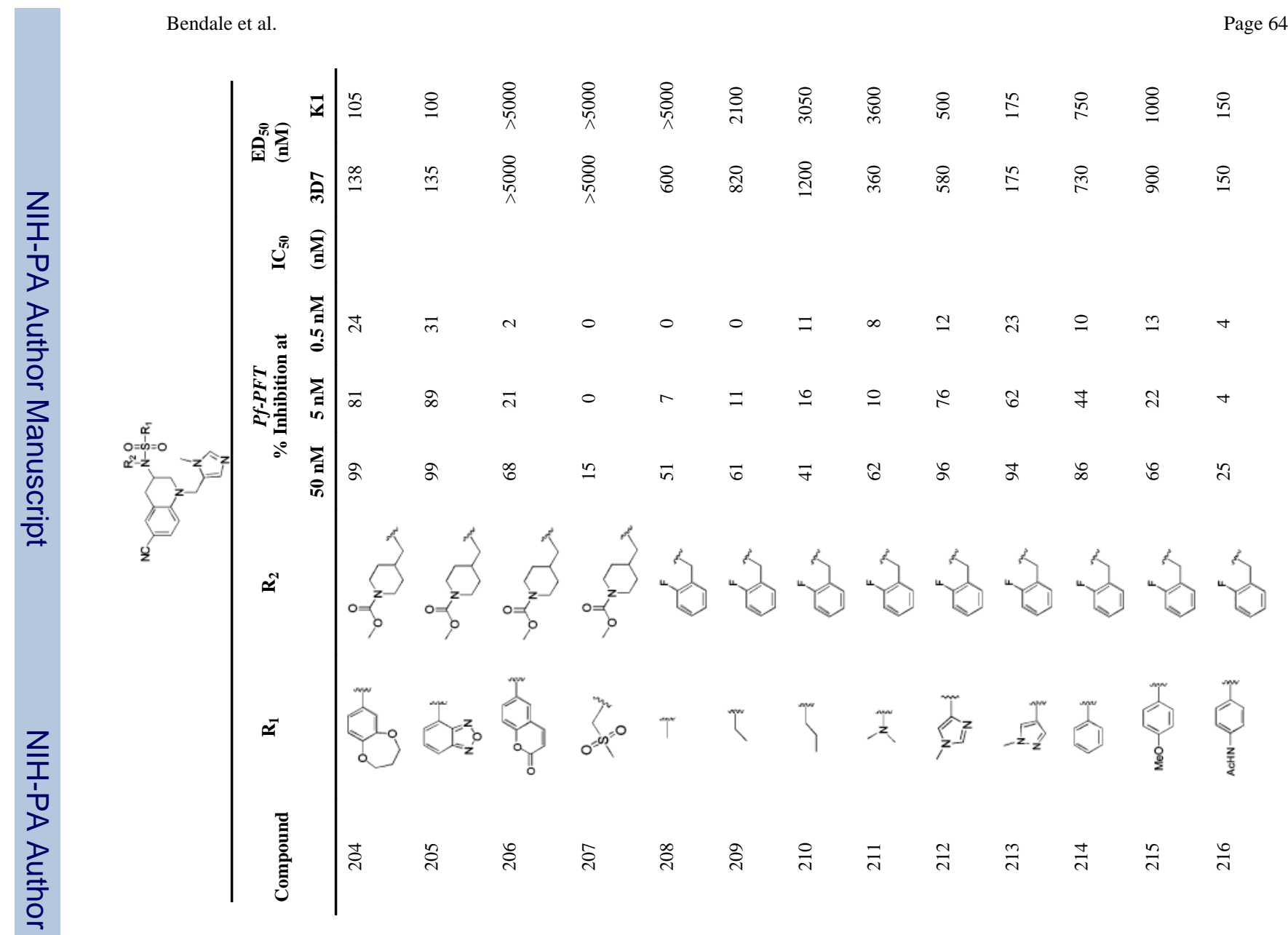




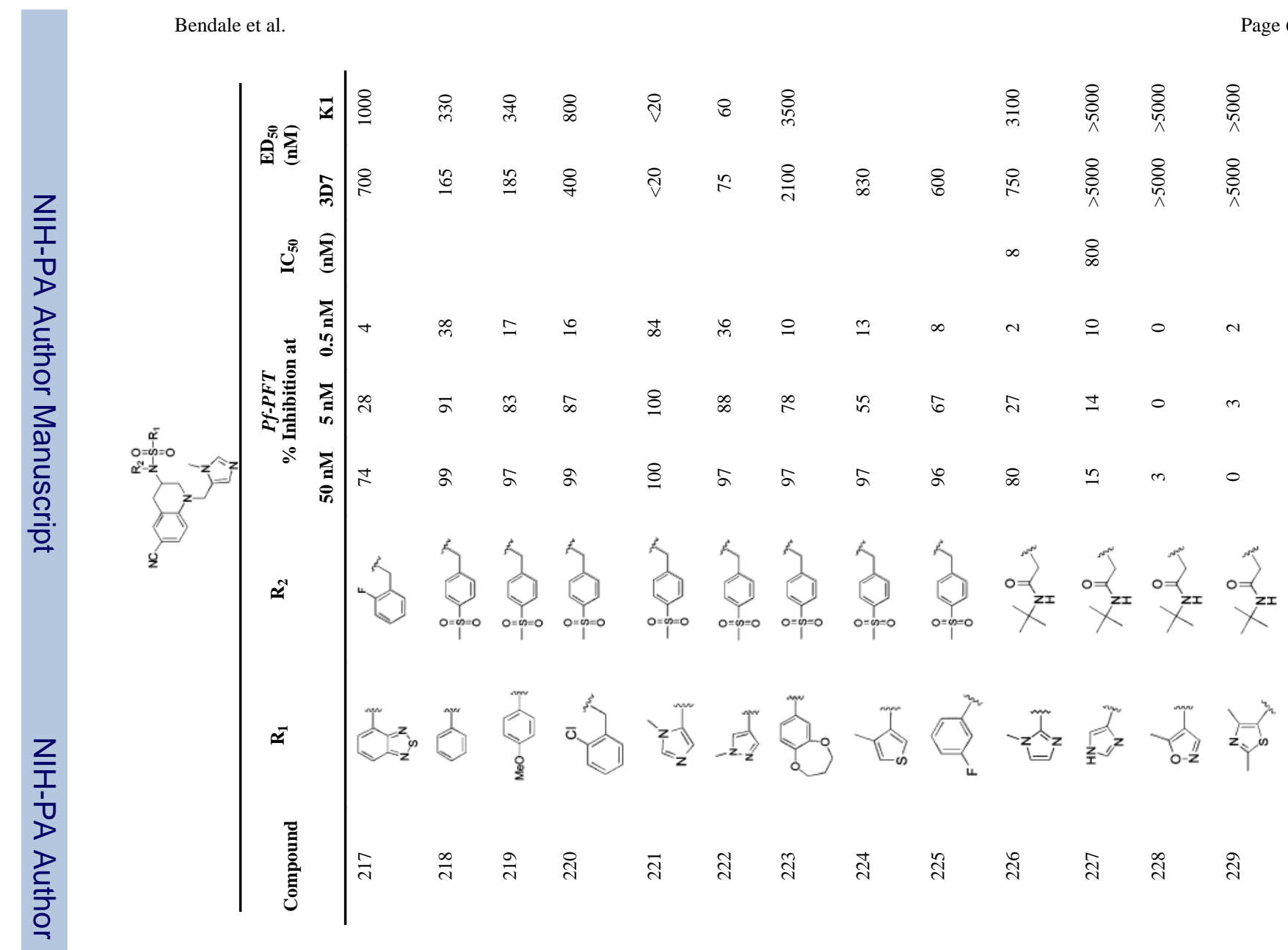




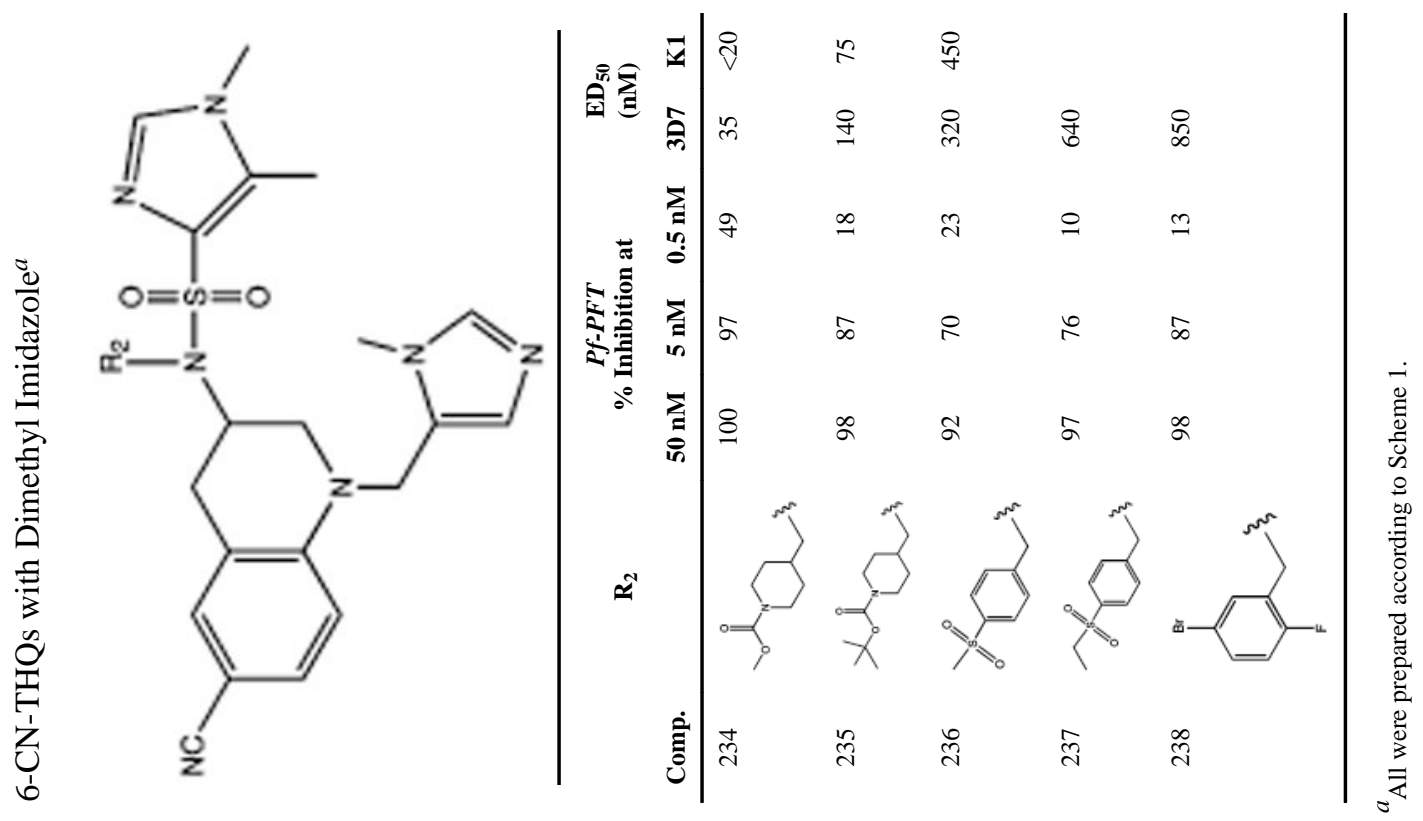

J Med Chem. Author manuscript; available in PMC 2010 June 30. 


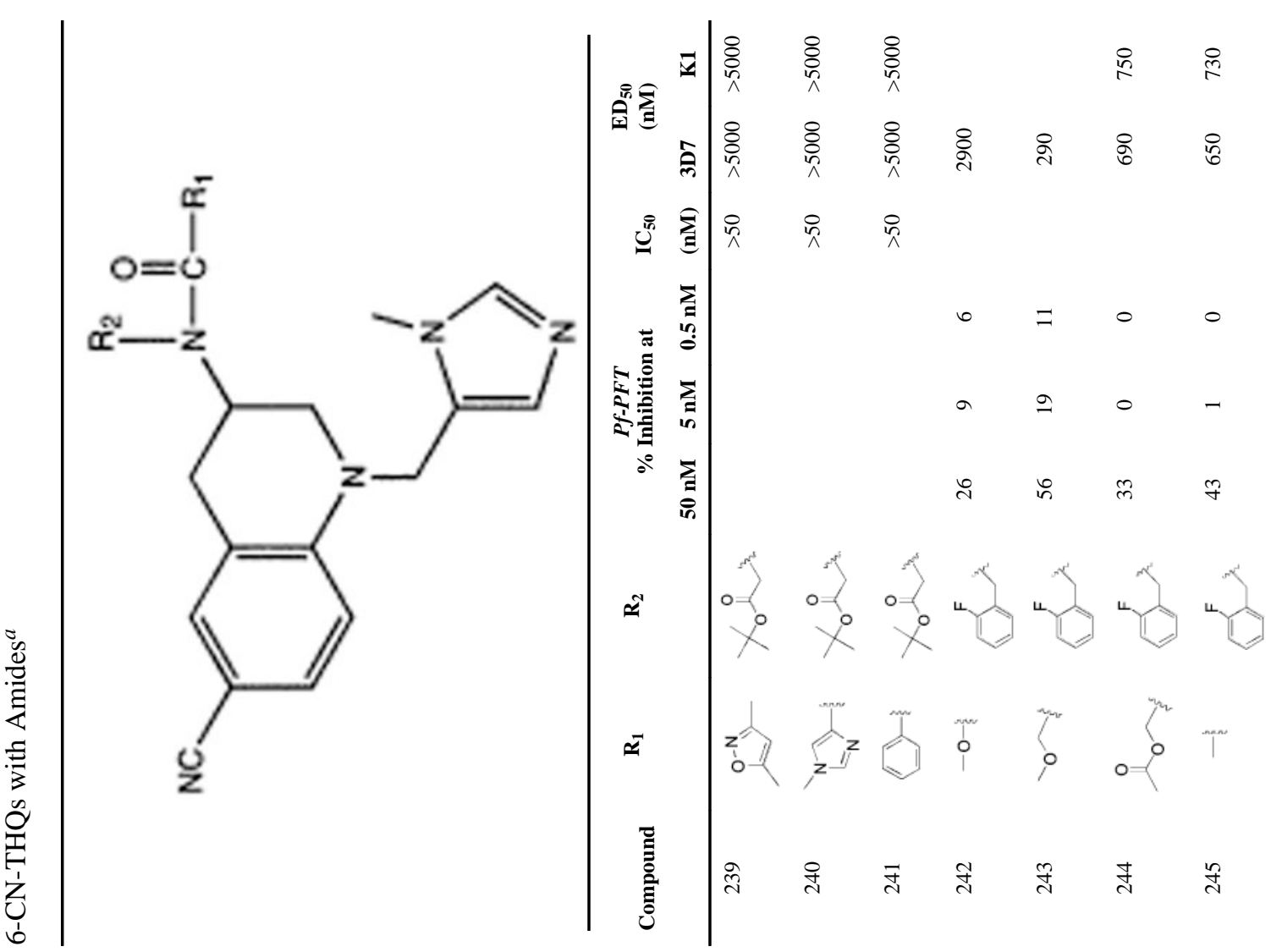

J Med Chem. Author manuscript; available in PMC 2010 June 30. 


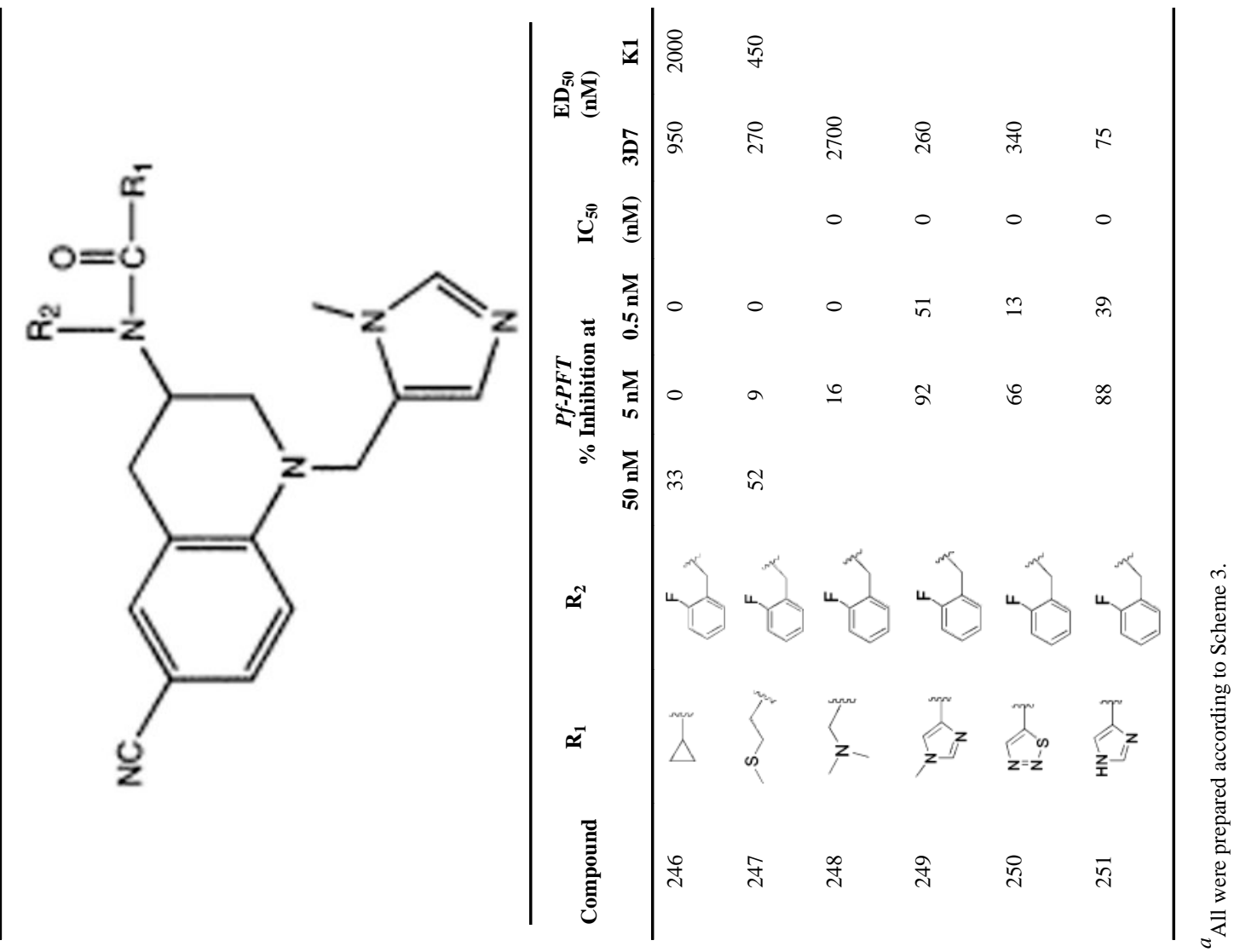




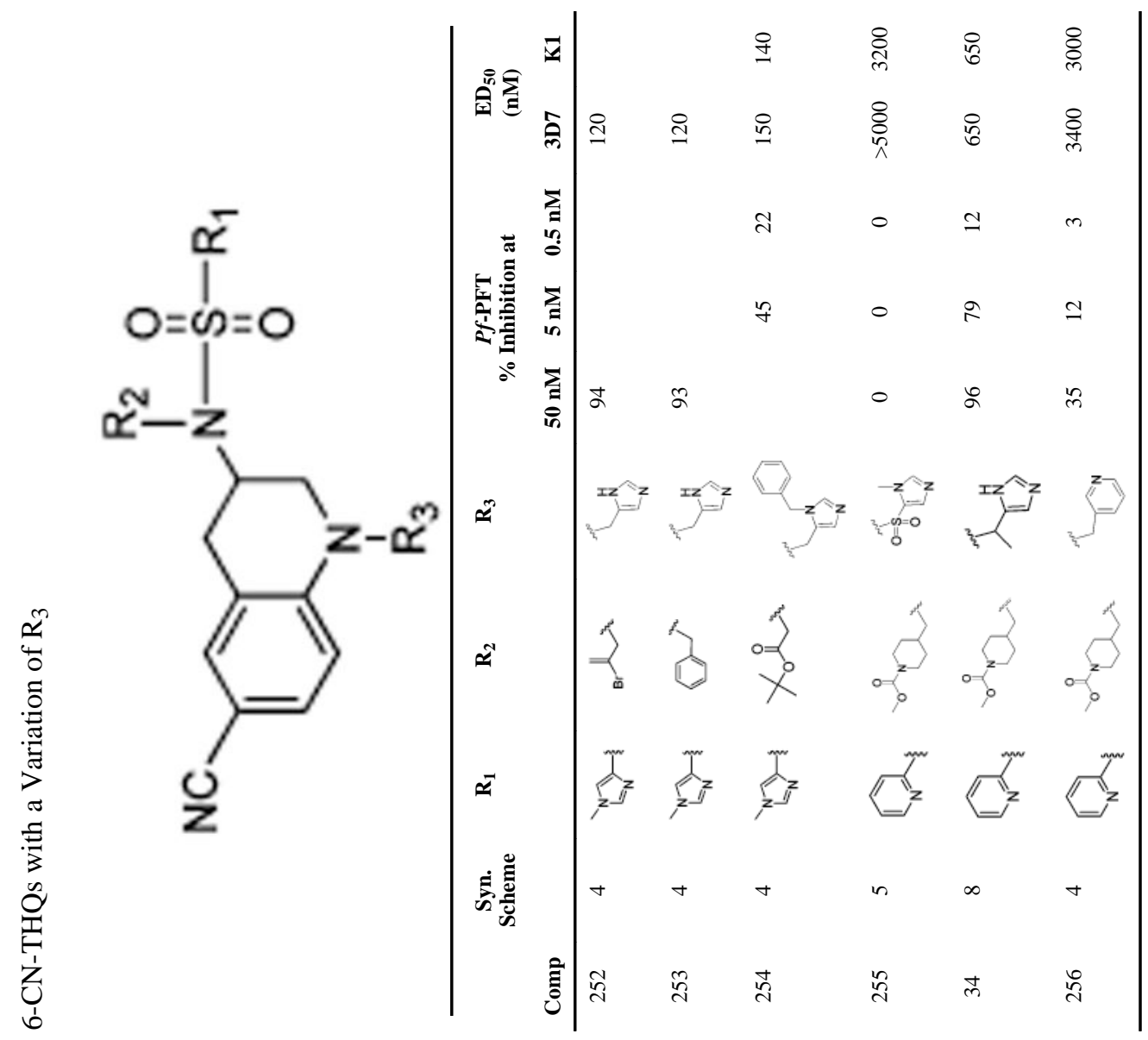




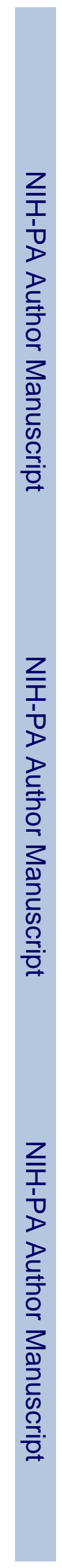

Bendale et al.

Page 72

ב⿱丶万一⿻⿻一𠃋十

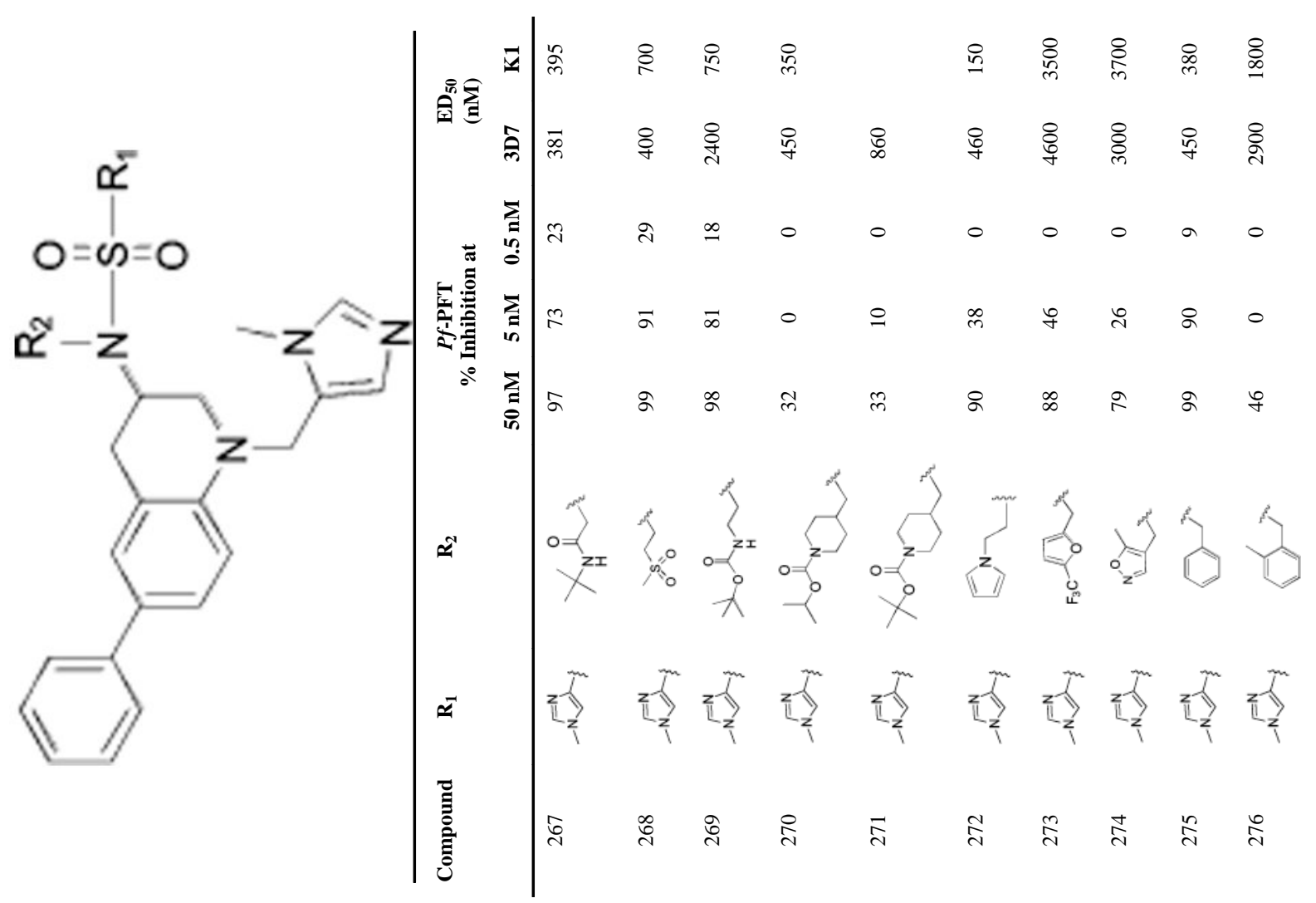

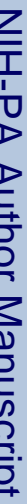

J Med Chem. Author manuscript; available in PMC 2010 June 30. 


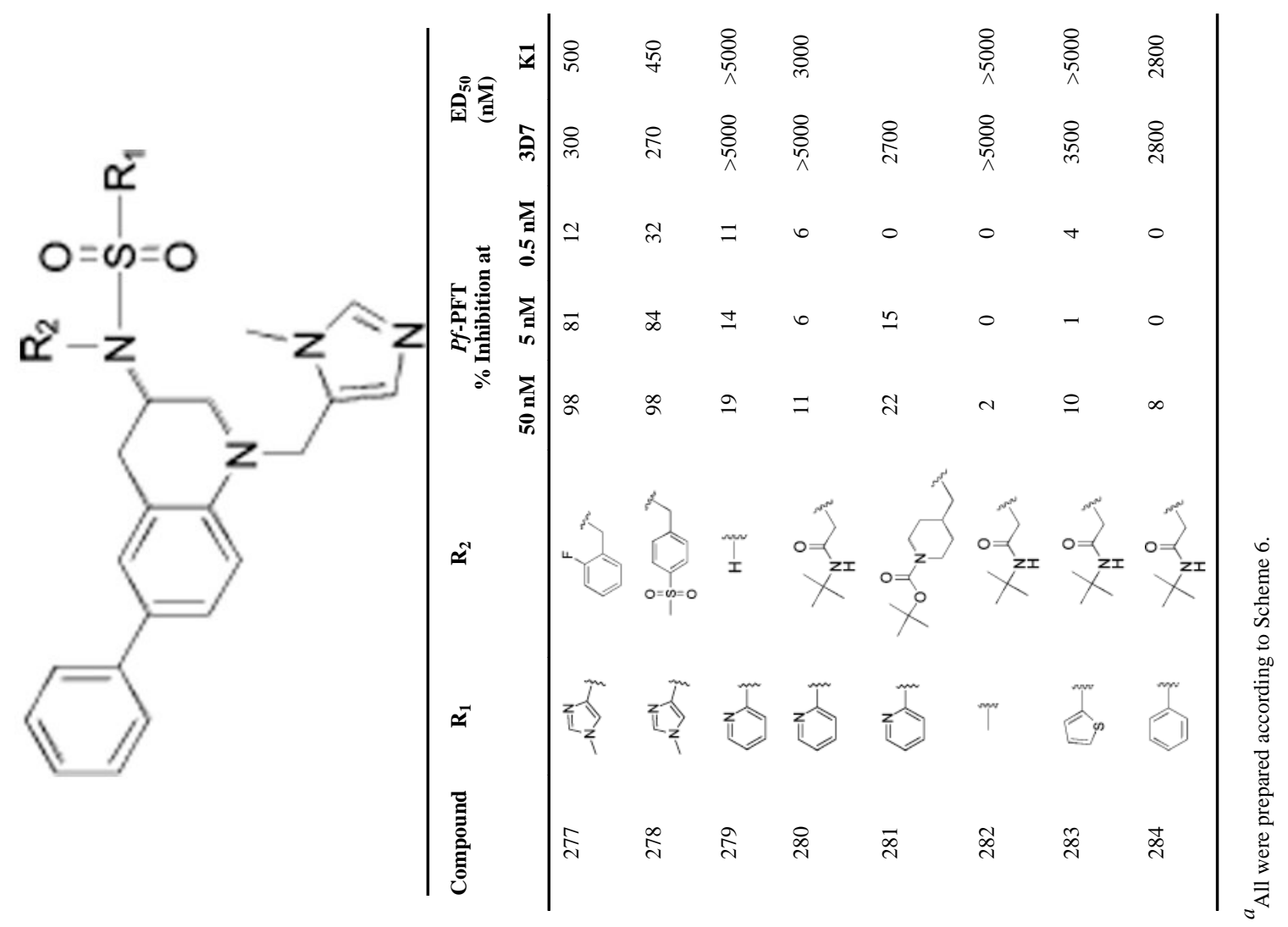

J Med Chem. Author manuscript; available in PMC 2010 June 30. 


\section{Table 10}

Potency on Mammalian versus Pf-PFT

\begin{tabular}{lll}
\hline cmpd & $\begin{array}{l}\mathbf{I C}_{\mathbf{5 0}} \text { on mammalian } \\
\text { PFT } \\
(\mathbf{n M})\end{array}$ & $\begin{array}{l}\text { Approximate } \\
\text { IC }_{50} \text { on Pf-PFT } \\
(\mathbf{n M})\end{array}$ \\
\hline 81 & 10 & 50 \\
65 & 3.8 & 2 \\
84 & 440 & 50 \\
55 & 1.7 & 0.2 \\
62 & 3 & 0.5 \\
6 & 7 & 2 \\
226 & 5 & 20 \\
151 & 7.8 & 2 \\
152 & 6.5 & 4 \\
162 & 3.2 & 0.4 \\
158 & 16 & 4 \\
167 & 25 & 3 \\
165 & 5.5 & 0.5 \\
134 & 2.6 & 0.5 \\
129 & 1000 & 40 \\
91 & 7.5 & 2 \\
101 & 105 & 40 \\
126 & 4.8 & 40 \\
123 & 3.4 & 1 \\
\hline & & \\
\hline
\end{tabular}

${ }^{a}$ IC50 values were estimated based on the $\%$ inhibition of Pf-PFT at $0.5,5$, and $50 \mathrm{nM}$ inhibitor using the data in Table 1-Table 9. 